
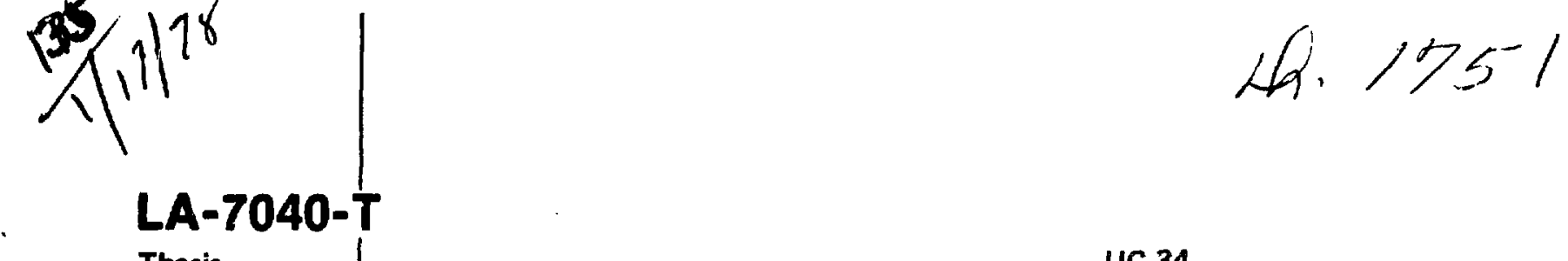

Thesis

UC-34

Issued: November 1977

\title{
Turbulent Diffusion of Small Particles
}

\author{
Leonard Gerald Märgolin
}




\title{
TURBULENT DIFFUSION OF SMALL PARTICLES
}

\author{
by \\ Leonard Gerald Margolin
}

A dissertation submitted in partial fullfillment

of the requirements for the degree of

Doctor of Philosophy

(Physics)

in The University of Michigan

1977

Doctora1 Committeo:

Francis H. Harlow, Los Alamos Scientific

Laboratury, iro-Chairman

Associate Professor David N. Williams,

Co-Chairinan

Professor Marc Ross

Professor Yukio Tonozawa

Professor William Williams

Professor William Wil:marth

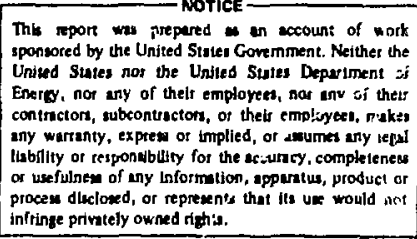

proces dieclond, or reprements, appuntus, product or

infringe privitely owred rights. 
NOTATION . . . . . . . . . . . . . . . . . . . . . . vi vi ABSTRACT . . . . . . . . . . . . . . . . . . . . . . vii INTRODUCTION . . . . . . . . . . . . . . . . . . . . . . . 1 CHAPTER I . . . . . . . . . . . . . . . . . . . . . . 19

1.1 Description of the Problem . . . . . . . . . . . . 19

1.2 The Diffusion Coefficient . . . . . . . . . . . . 23 Chapter II . . . . . . . . . . . . . . . . . . . . . 28

2.1 Momentum Exchange in TwoPhase Flow . . . . . . . . . . . . . . . 28

2.2 The Force on a Particle . . . . . . . . . . . . 30

2.3 App1ications to Bubbles
and Droplets. . . . . . . . . . . . . . . 36

CHAPTER III . . . . . . . . . . . . . . . . . . . . 37

3.1 The Method of Rice Applied to

the Simplified Equation of Motion . . . . . . . . . . 37

3.2 The Direct Integral Method . . . . . . . . . . . 41

CHAPTER IV . . . . . . . . . . . . . . . . . . . . . 60

4.1 Formal Solution of the Equations of Motion . . . . . . . . . . . . . . . . 60

4. 2 Comments on the Formal Solution . . . . . . . . . . 71

4.3 Calculation of Particle Diffusivity with Basset Forces... . . . . . . . . . . . 73

4.4 Evaluation of Particle Diffusivity
for Several Special Cases . . . . . . . . . . . . . 84

CHAPTER V . . . . . . . . . . . . . . . . . . . . . 88

5.1 Introduction of Shears into the Equation of Motion .. . . . . . . . . . . . . 88

5.2 The Viscous Forces . . . . . . . . . . . . . . . 107

5.4 The Inviscid Pressure Forces . . . . . . . . . . . . 111 
6.1 Solution of the Fiuations of

Motion with Shears................ . 119

6.2 Particle Diffusivity in the

Presence of Mean Flow Shears . . . . . . . . . . . 122

6.3 Comments on Fluctuating Shears . . . . . . . . . . . . 124

CHAPTER VII

7.1 Linearized Form Drag . . . . . . . . . . . . 131

REFERENCES . . . . . . . . . . . . . . . . . . . . . . . . 142

ACKNOWLEUGEMENTS . . . . . . . . . . . . . . . . . . . . 144 
NOIATION

\begin{tabular}{|c|c|}
\hline$D(t)$ & Time dependent particle diffusivity \\
\hline$D_{F}$ & Passive scalar diffusion coetficient \\
\hline$\ell_{\mathrm{C}}$ & Spatial correlation length \\
\hline$\mu$ & Dynamic viscosity \\
\hline$v$ & Kinematic viscosity \\
\hline$\psi$ & Pressure \\
\hline$R(t)$ & $\begin{array}{l}\text { Correlation of fluid velocity along the } \\
\text { motion of the particle }\end{array}$ \\
\hline$R_{0}$ & Particle radius \\
\hline$\rho_{F}$ & Microscopic fluid density \\
\hline$\rho_{P}$ & Mirroscopic particle density \\
\hline$\sigma_{i j}$ & Shear tensor \\
\hline$\sum_{i j}$ & Stress tensor \\
\hline$t$ & Time \\
\hline$t_{I}$ & Lagrangian integral scale \\
\hline$\vec{u}$ & Fluid velocity \\
\hline$\vec{b}$ & Particle velocity \\
\hline $\overrightarrow{\mathrm{x}}$ & Position vector \\
\hline$i$ & $i^{\text {th }}$ component of position vector \\
\hline
\end{tabular}


ABSTRACT

TURBULENT DIFFUSION OF SMALL PARTICLES

by

Leonard ¿Eıald Margolin

Co-Chairman: Francis H. Hariow, David N. Williams

The diffusion of small, spherical, rigid particles suspended in an incompressible turbulent fluid, but not interacting with each other, is studied. As a stochastic process, the turbulent fluid velocity field is assumed to be homogeneous, isotropic and stationary.

The assumptions on the particle force laws, as is usual in this kind of study, are fairly restrictive. Host of our discussion as;sumes the Stokes regime; and we begin with a particle equation of motion which includes only the effects of Stokes drag and a virtual mass force. For this simple force law we find an exact solution for the particle velocity correlation function, for all times and initial conditions, in terms of a fluid velociey correlation function measured along the motion of the particle. We show that for times larger than a certain time scale, the particle velocity correlation becomes stationary, and agrees with that found by Liu, who used a different method and assumed a stationary particle velocity correlation that the outset. This mears that if, as in the model of Tchen, the particle remains in the same neighborhood of fluid for times less than or on the order of the Lagrangien integral scale, so that the integrals over time of the Lagrangian correlation and the correlation of fluid velocities along the motion of the particle coincide, then the 
long time particle diffusivity in this model is the same as the passive scalar diffusivity.

Next, we include Basset and gravitational buoyancy forces on the particle, still in the Stokes regime. We are still able to solve for the exact particle velocity correlation in terms of the correlation of Eluid velocities along the motion of the particle, for all times and initial conditions. Under the same conditions as above, the long time particle diffusivity remains the same as the passive scalar fluid diffusivity.

Then, we consider the effect of small shears in the fluid velocity, under the additional restrictions of a certain high frequency regime for the turbulence, and that the shears convect past the particle much faster than the grovth of the boundary layer. New force terms due to the presence of such shears are calculated and incorporated into the equation of motion. A perturbation solution to this equation is constructed, and the resultant particle velocity correlation function and diffusion coefficient are calculated. To lowest order, the particle diffusivity is found to be unaltered by the presence of small, mean flow shears.

The last model we treat is one in which particles traverse a turbulent fluid with a large mean velocity. Among other restrictions, we assume linearized form drag. We calculate the diffusion coefficient for such particles and find it to be much smaller than the passive scalar diffusion coefficient, and we find agreement within five percent with the experimental results of Snyder and Lumley. 


\section{INTRODUCTION}

The study of the motion of small particles in a turbulent fluid is important in many situations. There are applications in describing the dispersal of pollutants in the atmosphere and in large bodies of water. In meteorology, there are applications toward understanding the physics of rainstorms and snowstorms. There are engineering applications in two-phase flow systems - for example, in understanding the effects of bubbles in the conlants of reactors.

The study of the general motion of a particle in a turbulent fluid is an extremely difficult task. We shall restrict our attention to small particles, meaning particles whose dimensions are much smaller than the smallest scale lengths of the turbulence - i.e., particles much smaller than the turbulent eddies. Even then, it isill be necessary to make a number of additional restrictive assumptions so that our soluticns, while significantly more extensive than previous ly available, are nevertheless far from covering a11 possible cases of particulate mot:son in turbulent flows.

In general terms, the problem may be considered to consist of two parts. Given a small particle in a turbulent fluid, we must first derive an equation of motion for the particle and construct a formal solution. The particle equation of motion will require, among other things, detailed jinformation about the flow field around the particle. However, in a tur julent fluid, this information is known 
only in a statistical sense. Thus, the second part of the problem will involve manipulating the formal solution of particle motinn to yield information about the statistics of particle motion.

The study of particle motion in a turbulent fluid is similar in many respects to the study of Brownian movement. ${ }^{1,2}$ Both depend upon the solution of a particle equation which contains a stochastic force and also terms which represent a frictional force opposing the forced motion. In each case, one finds a formal solution to the equation of motion, and is then able to relate the statistics of the particle motion to the statistics of the fluid. As is the case in Brownian movement, our principal task will be the calculation of a particle diffusion coefficient; For this reason, our problem is often called the turbulent diffusion of small particles.

There is an important difference between Brownian movement and the turbulent diffusion of small particles. This difference can be understood in terms of the comparison of two time scaies which characterize each problem. The first is the particle resfonse time which is a time scale associated with the frictional part of the equation of motion. For example, if the particle were given an initial velocity relative to the fluid, then, in the absence of any stochastic force, this relative velocity would immediately begin to decay to zero. The particle response time is the time required for the relative velocity to decay to some small, specified fraction of its initial value. The second time scale is the correlation time associated with the stochastic force. In Chapter II, we will define the correlation func- 
tion of a stochastic process, which is a measure of the statistical relationship of the stochastic variable at two times. The (normalized) correlation function of a stationary process is a function of the difference of the two times, which equals one when the two times are the same (perfect correlation), and which dies away to zero as the two times become more and more separated. In these terms, one may define the correlation time as the time interval for which the correlation function equals some smal1, specified fraction of one.

In Brownian movement, the stochastic force represents the effect of collisions of the fluid molecules with the particle. Each indiviual collision has relatively little effect on the particle, for the particle mass is much greater than the mass of a fluid molecule. Furthermore, it is assumed that each collision is a random event. Under these circumstances, the particle response time is much greater than the correlation time of the stochastic force. The process of Brownian movement can then be characterized as a Markoff process, ${ }^{2}$ and a Fokker-Planck equation ${ }^{2}$ can be used to solve for the particle velocity probability distribution.

The situation is more complicated in the turbulent diffusion of particles. Here the particle moves due to the drag of the surrounding fluid. The drag depends, among other things, on the fluid veiocity. In a turbulent fluid, one can think of an eddy as the region of fluid over which fluid velocities are spatially correlated. Thus, the correlation time of the stochastic force is like the time the particle remains in an eddy. This time can be estimated in two ways, in terms 
of the size of an eddy and the relative velocity of the particle with respect to the fluid, or in terms of the lifetime of an eddy. In general, it is not possible to assume that either of these times is much smaller than the particle response time. Thus. one sannot treat the turbulent diffusion of particles as a Markoff process.

In 1947 Tchen $^{3}$ made the first, extensive, theoretical study of the turbulent diffusion of small particles. Most of the later work on this problem, including this thesis, is closely related to that of Tchen. In the next few paragraphs, we will examine Tchen's theory. Then we will describe how succeeding investigators have modified and generalized this theory. With this basis, we can then describe the direction and scope of the new results of this thesis.

In Tchen's tiheory, the following assumptions are made:

1) The turbulence is statistically homogeneous and isotropic in space, and is stationary in time.

2) The fluid is ir inite in extent.

3) The particle is spherical and rigid.

4) The particle is small compared with the smallest spatial scales characterizing the turbulence.

5) The particle Reynolds number is much less than one. 6) The density of particles is sufficiently small that the effects of particle-particle interactions may be neglected.

7) Tchen's neighborhood hypothesis; as staced by Tchen, ${ }^{3}$ p.86, it is assumed that "the elements of the liquid which 
can be considered as moving with a practically homogeneous velocity are large not only compared with the dimencions of the suspended particles, but also with the paths described by the particles relatively (SiC) to the liquid."

The role and meaning of the neighborhood hypothesis is a very interesting and controversial subject, and we will examine it in great detail short $1 \mathrm{y}$.

of the assumptions listed above, the first six are employed to derive the particle equation of motion. By virtue of assumption six, the effect of particle-particle interactions is neglected. The force of the fluid on the particle, in principle, is determined by the flow around the particle. The flow, in turn, is determined by the NavierStokes equations. In practice, there are few situations for which the Navier-Stokes equations, with appropriate initial and boundary conditions, can be explicitly solved. With the first five assumptions, Tchen restricts himself to the Stokes regime in which the BassetBoussinesq-Oseen ( $B-B-O$ ) equation of motion for the particle is applicable.

It should be noted here that assumption five does not necessarily restrict the size of the particle. The particle Reynolds number is the product of the relative velocity of the particle with respect to the fluid, and the particle radius, divided by the fluid viscosity. In Chapters III and IV of this thesis, we will derive expressions for the relative velocity in this regime, and show that it is proportional to the difference of particle and fluid density. Thus, for any 
choice of particle radius, therc will be a range of particle densities for which the particle Reynolds number is much less than one. We also note, at this point, that assumption four does explicit ty restrict the size of the particle. In turbulent flows, the smallest spatial scale of the turbulence is the Kolmogoroff microscale, which is a measure of the size of the sinallest, energy-dissipating eddies. Assumption four states that the particle must be much smaller than the Kolmogoroff nicroscale.

Having justified the use of the $B-B-O$ equation of motion for the particle, Tchen constructs a formal solution for the particle velocity. This solution is written as a convolution over the fluid velocity and its first two derivatives (Tchen, ${ }^{3} \mathrm{p} .81$ ). Now there are several points we must emphasize about Tchen's formal solution for particle motion. First of all, Tchen's formal solution is valid only in a restricted regime where the particle density is larger than 1.75 times the fluid density (see Chapter IV of this thesis). Furthermore, even within tnis range of densities, Tchen's solution is only asymptotically correct in the limit in which the initial conditions of particle and fluid velocity are forgotten - i.e., for problem times much greater than the particle response time. Finally, we point out that the fluid velocity in terms of which the solution for particle velocity is written is the fluid velocity measured at the position of the particle. This fluid velocity is not simply a property of the fluid, but also depends on the motion of the particle.

With the formal solution for particle velocity, Tchen then calculates the particie diffusion coefficient. This diffusion coefficient 
devends upon the integral over time of the correlation function of fluid velocity. However, we again emphasize that these fluid velocities are measured at the particle, and so the correlation functions must be measured along the path of the particle. Now in order to relate the statistics of particle motion (i.e., the particle diffusion coefficient) to the statiscios of the fiuid, it is necessary to take one further step. This step will involve expressing the correlation of fluid velocities along the motion of the particle in terms of the correlation of fluid velccities measured in some manner independent of the presence of the particle. Thus, at this point, Tchen first employs assumption seven, the neighborhood hypothesis. The purpose of this hypothesis, as stated by Tchen, is to identify the correlation of fluid velocities measured along tile path of the particle with the Lagrangian correlation - that is, with the correlation of flufd velocities measured along the motion of a fluid element.

Now these two correlation functions can become the sane only in the limit that the particle becomes an element of fluid, that is, when particle density equals fluid density, and the particle radius becomes very smal1. In this limit, the particle diffusion coefficient is the fluid, or passive scalar diffusion coefficient. For particles which are not fluid elements, the neighborhood hypothesis cannot be exactly correct. The question we now address is, under what circumstances, if any, does such a hypothesis represent a reasonable approxImation?

Let us define a neighborhood to be a region of fluid over which 
the fluid velocity, at any given instant of time, may be assumed to be nearly homogeneous. With this definition, we can restate Tchen's neighborhood hypothesis as follows (Hinze, ${ }^{4}$ p. 461) - "during the motion of the particle, the neighborhood will be formit by the ame fluid." If the particle has any mean square velocity relative to the fluid - i.e., if the particle is not an element of fluid - then eventually it must leave its original neighborhood of fluid. It is not reasonable to assume that Tchen's neighborhood hypothesis is valid throughout the motion of the particle.

However, Tchen's statement of the neighborhood hypothesis is much more restrictive than is actually required. What is required is that the integrals over time of the correlation functions of fluid velocity, one measured along the path of the particle, and one measured along the path of a fluid element, are equal. Toward this end, we reformulate the neighborhood hypothesis as follows:

It is assumed that the particle remains in the same neighborhood of fluid for time intervals of the order of the fluid correlation time.

The fluid correlation time here refers to the correlation time associated with the Lagrangian correlation; this time is also called the Lagrangiar integral scale (see Hinzo, ${ }^{4}$ p.53). Hereafter, when we refer to the neighborhood hypothesis, we shall mean our statement, and not Tchen's statement.

We examine the validity of the neighborhood hypothesis based on two ideas. First, does the same fluid form the neighborhood during 
the period of a correlation time? Second, does the particle remain in the neighborhood during the period of a correlation time?

The first question is concerned with the idea of volume stretching. Figure 79 on p.492 of Monin and Yaglom portray.s this idea, showing the time variation of a volume of fluid that is initially nearly spherical, and that is stretched and distorted into long thin ribbons. The concern is that these ribbons become too smal1 to be considered neighborhoods of the particle, However, within the framework of Tchen's fourth assumption, this can never happen. The point is that the stretching process dces not create ever smaller and smal1er ribbons. Instead, the smallness of the ribbons is definitely limited to be at least as large as the smallest scales of the turbulence, for the stretching is itself a turbulent process. Now Tchen's fourth assumption states that the particle is much smaller than the smallest scales of the turbulence. Thus, any of the ribbons, although much smaller than the original volume, is still large enough to constitute a neighborhood of the particle if it contains the particle.

The second question is concerned with the relative velocity of the particle with respect to the fluid. We expect that the neighborhood hypothesis will be a good approximation whenever this relative velocity is much smaller than the size of a neighborhood divided by the fluid correlation time. In Chapter III of this thesis, we shall examine this idea quantitatively, deriving specific conditions under which the neighborhood hypothesis may be considered reasonable. These conditions are that the particle density must be very nearly equal to the fluid density, or that the particle response time is much smaller 
than the fluid correlation time. When elther of these conditions is realized, the particle will spend most of the time accommodated to the fluid, and the relative velocity of the particle with respect to the fluid will be small.

To sumarfze, there is a restricted class of particles for which our revised statement of the neighborhood hypothesis is justifiable. For particles in this class, when Tchen's first six assumptions are valid, and for problems whose duration is much greater than the particle response time, the particle diffusion coefficient will equal the passive scalar dfffusion coefficient. We have not found any experimental data concerning the turbulent diffusion of particles for which our revised neighborhood hypothesis is justifiable. This point will be discussed later in this introduction when we review the experimental work on the turbulent diffusion of particles.

In 1956, Liu ${ }^{6}$ offered an alternate method for treating the turbulent diffusion of sma11 particles. Liu's treatment is based on the method of generalized harmonis analysis, and yields a particle diffusivity in a very elegant, yet simple manner. Liu's theory is based on the same particle equation of motion that Tchen uses. Furthermore, Liu states on p.403 - "it is well-known (Dryden, 1939; Batchelor, 1949) that when the diffusion time is very large, the turbulent diffusion of a given initial distribution of marked fluid in a homogeneous, isotropic turbulent field can be closely represented by a Fickian-type diffusion equation with the diffusion coefficient proportional to the integral on the left side of (33)." However, the left side of (33) is the 
integral of the fluid correlation measured along the path of the particle, not the integral of the Lagrangian correlation. As we have a1ready discussed, the diffusion coefficient of marked fluid (i.e., the passive scalar diffusion coeffirient) is proportional to the integral of the Lagrangian correlation. Thus, Liu has implicitly assumed the equality of the integrals over the two correlation functions, and so implicitly made use of an assumption equivalent to the neighborhood hypothesis. Liu continues on p.403 to say "the conciusion represented by (33) therefore justifies use of the same diffusion equation to prescribe the disfarsion of the particles as well as the marked fluid. It should be noted that a result similar to (33) has been previously obtained by Tchen (1947) by a different method."

Liu ${ }^{6}$ also calculates the mean square displacement as a function of time. In particular, he is able to isolate the time dependence in the limits where the time is much greater than, or much less than, the fluid correlation time. We should note that Liu's method assumes that the particle velocity is a statistically stationary function, and so is only valid for times when the effect of initial conditions of particle and fluid velocity are forgotten. Thus, while investigating the limit of times short compared with the fluid correlation time, Liu must still restrict himself to times much longer than the particle response time. The later work of Hinze ${ }^{4}$ and Levich ${ }^{7}$ is closely related to that of Liu. ${ }^{6}$ Friedlander ${ }^{8}$ also develops a similar expression for the mean square displacement, in the short time limit, but based on Tchen's formalism. 
Many authors have discussed the particle equation of motion in a turbulent fluid. Corrsin and Lumley ${ }^{9}$ generalize the equation of motion to include terms involving the spatial derivatives of fluid velocity. Hinze ${ }^{10}$ discusses additional particle-fluid interaction forces such as form drag, Magnus force, and the Saffman force, estimating their size and describing situations in which these forces may be important. Lumley ${ }^{11}$ discusses the difficulties involved in treating non-spherical particles, non-Newtonian flows, and problems where the particles are closely packed. Hinze ${ }^{10}$ discusses the particle-particle interaction in turbulent flows, and also the effect of the particles on the turbulence. Peskin ${ }^{12}$ has modeled the particle-particle interaction and calculated an additional particle diffusivity due to this interaction. Ahmadi and Goldschmidt ${ }^{13}$ have considered the case where the particle is comparable in size to the Kolmogoroff microscale. They hypothesize a filtering effect of the particle on the fluid turbulence spectrum. Their theory predicts that the particle jiffusivity may exceed the passive scalar diffusivity. Hinze ${ }^{10}$ describes a simple filtering effect model in which the motion of a neutrally buoyant particle is not affected by eddies with a wavelength smaller than some cut-off value. Plotting the ratio of particle to fluid diffusivity versus the cut-off wavelength, Hinze finds this ratio reaches a value slightly larger than one when the cut-off wavelength is about one half the Kolmogoroff microscale. For larger values of the cut-off wavelength, the diffusivity ratio drops sharply.

Lumle ${ }^{14}$ investigated the problem of predicting the particle diffusivity without using the neighborhood hypothesis. This problem 
involves some very difficult mathematics, Concerning this work, Lumley cumments in a later paper" 15 "Lunley concluded that the problem can be resolved only on the level of functional probabilities. In essence, he says that, in order to predict the probability of a particle traveling Irom one point to another, one must integrate, in function space, over al1 possible paths between two points, the probability of the: particle taking the path. The theory of functions and function spaces is simply not yet develaped to a point where such a calculation could be carried out, although elementary steps have been made."

Peskin ${ }^{16}$ makes the assumption of a joint Gaussian distribution for fluid velocities at separate spatial points. In this case, the correlation of fluid velocities along the path of the particle equals the Lagrangian correlation reduced by the spatial correlation corresponding to the distance the particle has moved relative to the fluid. By assuming this distance remains sma11, Peskin is able to calculate a particle diffusivity slightly smaller than the passive scalar diffusivity.

Concerning the experimental work on the turbulent diffusion of particles, we refer to the paper by Snyder and Lumley. ${ }^{15}$ In this paper previous experimental work is discussed. The authors state, on p.44, that "previous experiments have failed to produce completely satisfactory data for numerous reasons." In their own experiment, Snyder and Lumley ${ }^{15}$ measure the mean square displacement of several types of particles as a function of time. The particles are diffusing through turbulent air. The results are plotted in Fig. 9 of the paper. 
For large times, the mean square displacement of each type of particle Is well represented as a linear function of time whose slope represents the particle diffusion coefficient (see Chapter I of this thesis). For each type of particle, the diffusion coefficient is much less than the passive scalar diffusion coefficient. Comparing the experimental conditions with the assumptions of Tchen's ${ }^{3}$ theory, one finds that all of Tchen's assumptions, with the exception of the neighborhood hypothesis and asumption five concerning the particle Reynolds number, are justifiable in the experiment. In the experiment, the particle Reynolds number ranges from .05 to 2.5 for the different types of particles. One cannot rigorously justify the use of the $B-B-O$ equation for Reynolds numbers as large as 2.5 , but, on experimental grounds, it appears reasorable to use the $B-B-O$ equation in these circumstances. This point is discussed further in Chapter II of this thesis.

In Snyder and Lumley's experiment, ${ }^{15}$ however, the particles falI under the influence of gravity. Since the ratio of particle to fluid density varies from 250 to 8900 , the particles acquire a large mean velocity relative to the fluid. In fact, this mean velocity is of the same order of magnitude as the r.m.s. fluctuating fluid velocity for one type of particle, and is much larger than the r.m.s. fluctuating fluid velocity for the other three types of particles. Now Snyder and Lumicy ${ }^{15}$ show that the r.m.s. fluctuating fluid velocity is approximately the spatial Eulerian integral scale divided by the Lagrangian time integral scale. Furthermore, the spatial Eulerian integral scale must be a good estimate of the maximum size of a neighborhood, for it 
is the length over which tine spotial correlation of velocities falls to a small fraction of one. Thus, the relative velocity of the particle with respect to the fiuid is comparable to, or much larger than, the size of a neighborhood divided by the fluid correlation time. As we have noted previously, in these circumstances, we cannot expect the neighborhood hypothesis to apply.

One can understand qualitatively why the measured particle diffusivities are less than passive scale diffusivity. On the basis of using the B-B-O equation of motion, Tchen's theory shows that the particle diffusivity is proportional to the integral over time of the correlation of fluid velocities along the motion of the particle. In the limit where the particle may be considered an element of fluid, the correlation function becomes the Lagrangian correlation and the diffusivity becomes the passive scalar diffusivity. Hinze ${ }^{10}$ points out that the correlation of fluid velocities along the motion of the particle should be less perfect than (i.e., is less than) the Lagrangian correlation. Hence, the particle diffusivity should be less than the passive scalar diffusivity. Furthermore, in Snyder and I.umley's experiment, the particles whose diffusivities are largest, are those whose density is closest to fluid density, and whose relative terminal velocity with respect to the fluid is smallest.

Let us now discuss the work in this thesis. We begin, in Chapter I, by describing the mathematical framework in which we treat the problem. We then drive an expression for the particle diffusiun coefficient, as a function of time, in terms of the correlation of particle 
velocities.

In Chapter Ii, we derive the parcicle equation of motion from the momentum equations of two-phase flow. This equation includes a term representing the particle-fluid interaction force. In the regime of small particle Reynolds number, when terms involving the spatial derivatives of fluid velocities may be neglected, this interaction force is known to be the sum of three terms - Stobes drag, the virtual mass term, and the Basset history term. Thus, in this regime, wh:-ch we call the Stokes regime, the particle equation of motion becomes the B-B-O equation used by Tchen. ${ }^{3}$ It is pointed out that the same expression for the particle-fluid interaction force, and hence the same particle equation of motion, is valid in a much different regime when the dominant frequencies of oscillation of the fluid are assumed large, and the amplitudes of oscillation of the fluid are small compared with particle dimensions. In this specialized regime, which we call the high-frequency regime, the particle Reynolds number may be very large. The assumption of a high-frequency regine would not be justified in the theoretical modelling of the experiment of Snyder and Lumley ${ }^{15}$. However, the assumption of a high-frequency regime can be expected to be appropriate for certain particles in high frequency turbulent flows such as are found in wind tunnels and strongly churning water.

In Chapters III and IV, we calculate the particle velocity correlation function, and the particle diffusivity, as functions of time. These calculations are based on the B-B-O equation of particle motion, and on our revised neighborhood hypothesis. One important new aspect of our calculation is that the particle velocity correlation and the 
particle diffusivity are written as functions of time, and are valid for all times, including times much less than the particle response time. In addition, our solution of the $B-B-0$ equation is valid for all values of particle density. This is not case for Tchen's solution of the $B-B-O$ equation, which we have already noted is valid only for particle densities greater than 1.75 times the flujd density. Comparison of the time dependent diffusivity with the long time diffusivity calculated by Tchen $^{3}$ is made, allowing us to establish criteria for when the long time theory is applicable. Also, in Chapter III, a simple model is constructed which allows us to describe conditions under which use of our revised neighborhood hypothesis will be iustifiable.

We should point out that our expressions for the particle velocity correlation and diffusivity, are written in terms of the Lagrangian correlation of fluid velocities. The theory of turbulence is not presently at a point where the Lagrangian correlations are generally known. We can oniy reemphasize that our goal is to relate the statistics of particle motion to the statistics of the fluid, and is not to furnish a general theory of turbulence.

In Chapters V and VI, we modify the theory of Chapters III and IV in the following manner. We shall now assume that the fluid velocity in a neighborhood of the particle is nearly, but not exactly, homogeneous in space. We accomplish this by allowing for the presence of small shears in the fluid velocity. By small, we shall mean shears much less than the r.m.s. fluctuating fluid velocity, divided by the particle radius. One might think of our characterization of fluid velocity in terms of a Taylor expansion about $t$. . tion of the particle. In Chapters III and IV, we keep only the first term of this expansion. 
In Chapters V and VI, we keep the first two terms.

The presence of small shears in the fluid velocity will affect the theory of Chapters III and IV in two respects. First, it will alter the particle equation of motion. Second, it will modify the relationship between the Lagrangian correlation, and the correlation of fluid velocities along the motion of the paiticle. In Chapter $V$, we will 1 derive additional terms in the particle-fluid interaction force which are linear in the shears. Because of mathematical complexity, we will restrict ourselves to the high-Erequency regime described previously. In Chapter VI, we will construct a solution to the new particle equation of motion shich contains the lowest order correction due to the presence of small shears. From this solution, we shall construct a particle velocity correlation function and a particle diffusivity. These auantities will depend on the Lagrangian correlation, and also a trifle correlation function formed of two fluid velocities and the shear. Like the Lagrangian correlation, the triple correlation will depend only on fluid properties, and not on the presence of the particle.

In Chapter VII, we will consider the case where the particle has a large terminal velocity with respect to the fluid. This is a cas? where the neighborhood hypothesis does not apply at all. This case does describe the situation for three types of particles in the experiment of Snyder and Lumley. ${ }^{15}$ We shall construct a theory for this particle diffusivity in these circumstance. The predictions of this theory are found to agree within five precent with the measured data of Snyder and Lumley for these three types of particles. 
1. I Hescription of the Problem

Consider a parriche suspronded in al turbalent fluid. We shall assume our particle is a sphere of rablus $R_{0}$ and density op we further assume our fluid is incomprossihle; thus the fluid has constant density $\rho_{f}$ and the diverguner of its veloeity field vanishes. We shail want ro make several assumptions concerning the statislical natur. of the turbulence. First, however, it will bo useful to conalruet it statistical framework in which to operate.

The bethavior of the fluid is, all least in principle, completoly determined by the Navier-Stokess equation and the divergenes condition, provided that wo supply suitable boundary and initial conditions. When the fluid keynolds number is sofficiently small, we will indeed have a laminar flow governed hy these equations. For larger values of the Reynolds number, the flow becomes anstable to small perturbations. For example, such perturbations might be gencrated by very tiny irregilarities in the wisls of the sysit em. Rather than attempt to describe the sytitem in complote microscopic detail, wo adopt the following point of viow. We consicler an ensemble of systems, earh characterized by the same marroscopic boundary and initjal conditions, but anch difforing in microscopir detai1. In each system we wili rolize a different pattern of turbulence. The fluid volocity $\vec{u}_{\mathrm{F}}$ is now to bi considered a stochastic variable whose statistias are obtained by appropriate averaging over 
the entire ensemble. Ensemble averages of stochastic quantities will be indicated by bars over the quantities. For example, we might fix on one particular point $\vec{x}_{0}$ and time $t_{0}$ if we neasure $\overrightarrow{11}_{F}\left(\vec{x}_{n}, t_{0}\right)$ in each member of the ensemble and average all the mo zurements, we obtain $\vec{u}_{F}\left(\vec{x}_{0}, t_{0}\right)$ which is the mean fluid velocity at point $\vec{x}_{\sigma}$ and time $t_{0}$.

The fluid velocity may be written as the sum of two parts

$$
\vec{u}_{F}=\vec{u}_{F}+\vec{u}_{\vec{p}}
$$

where $\vec{u}_{p}$ is the mean valocity and $\vec{u}_{F}^{\prime}$ is the flactuating velocity. By definition $\vec{u},=0$. Introducing a particle into each system of the ensemble, we may decompose the partivle velority in the same manner. The equations of motion we shall consider in the first. six chapters are all. Iinear in the fluid and particle velocities. If we write the equation of motion in terms of the decomposition of each velocity field into its mean and fluctuating parts, and then take the ensemble average of this equation, we find $\vec{u}_{p}$ is not dependent on $\vec{u}_{F}$. Subtracting the ensemble-averaged equation from the original equation of motion shows that $\vec{u}_{p}^{\prime}$ is independent of $\vec{u}_{F}$. The diffusion is an effect of the fluctuating velorities and so, without loss of generality, we shall assume $\vec{u}_{\mathrm{v}}=0$ in the first six chapters of this work.

We shall be primarily interested in two-velocity averages of the form

$$
u_{F_{1}}\left(\vec{x}_{1}, t_{1}\right) u_{F_{1}}\left(\vec{x}_{2}, t_{2}\right) \equiv R_{1 i}\left(\vec{x}_{1}, t_{1}, \vec{x}_{2}, t_{2}\right)
$$


Here ${ }_{i}$ is the $i$ th component of fluid velocity and there is no summation of indices implied. Averages of this form are called volocity correlation functions. If we define a normalized concrelation Function by

$$
N_{j i}\left(\vec{x}_{1}, t_{1}, \vec{x}_{2}, t_{2}\right) \equiv \frac{\overline{u_{i}\left(x_{1}, t_{1}\right) u_{F_{i}}\left(x_{2}, t_{2}\right)}}{\sqrt{{ }_{{ }_{F_{i}}{ }^{2}\left(\vec{x}_{1}, t_{1}\right)} \sqrt{u_{F_{i}\left(\vec{x}_{2}, t_{2}\right)}}}}
$$

then it is easy to show 17
1) $N_{i j}\left(\vec{x}_{1}, t_{1}, \vec{x}_{2}, t_{2}\right)=N_{i i}\left(\vec{x}_{2}, t_{2}, \vec{x}_{1}, t_{1}\right)$
2) $\left|\mathrm{N}_{\mathrm{i} i}\left(\overrightarrow{\mathrm{x}}_{1}, \mathrm{t}_{1}, \overrightarrow{\mathrm{x}}_{2}, \mathrm{t}_{2}\right)\right| \leqslant \mid \mathrm{N}_{\mathrm{i} \cdot \mathrm{i}}\left(\overrightarrow{\mathrm{x}}_{1}, \mathrm{t}_{1}, \overrightarrow{\mathrm{x}}_{\mathrm{i}}, \mathrm{t}_{1}, \mid=1\right.$.

We shall. also assume

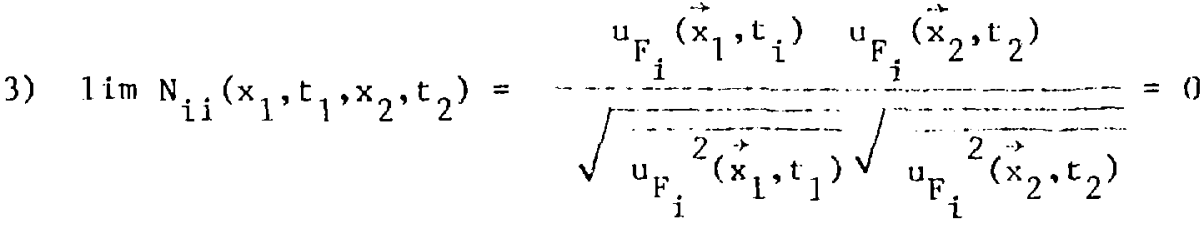

as

$$
t_{2}-t_{1} \rightarrow \infty
$$

or

$$
\left|\vec{x}_{2}-\vec{x}_{1}\right| \rightarrow x_{1}
$$

In words, the second property says that the velocity at any given place and time is perfectly correlated with itsclf. The correlation of velucities at different points and/or times is loss perfert. The third property says the velocities at widely separated times or points 
are nearly uncorrelated.

The Fluid correlation is a function of two spatial points $\vec{x}_{1}$ and $\vec{x}_{2}$ and $\mathrm{EE}$ two times $t_{1}$ and $t_{2}$. If the spatial dependence enters only through the combination $\vec{x}_{1}-\vec{x}_{2}$, we say the turbulence is homogenecus. If, th addition, we find the correlation depends on the space points only through the combination $\left|\vec{x}_{1}-\vec{x}_{2}\right|$, we say the turbulence is homogeneous and isotropic. Finally, if the correlation depends on the time variables only Lhrough the combination $t_{1}-t_{2}$, we say the turbulence is stationary. We shall assume our turbulent fluid is homogeneous and isotropic: and stationary.

The correlation $R_{i i}\left(\vec{x}_{1}, t_{1}, \vec{x}_{2}, t_{2}\right)$ is a very general form. By imposing restrictions on the arguments we derive more sperialized forms. For example, setting $t_{1}=t_{2}=t$, we derive the spatial correlation function $R_{i j}\left(\vec{x}_{1}, \vec{x}_{2}, t\right)$. In the same vein, we can allow $\vec{x}_{2}$ to depend parametrically on tile time and on $\dot{x}_{1}$. The simplest dependence is $\vec{x}_{2}=\vec{x}_{1}$ which yields $\mathrm{R}_{\mathrm{ii}}\left(\overrightarrow{\mathrm{x}}_{1}, \mathrm{t}_{1}, \mathrm{t}_{2}\right)$, the Eulerian time correlation. If $\vec{x}_{2}(t)$ describus the trajectory of an infinitesimal element of fluid located at $\dot{x}_{1}$ at time $t_{1}$, we have the Lagrangian time correlation. If $\vec{x}_{2}(t)$ describes the trajectory of a particle suspended in the fluid which is located at $\vec{x}_{1}$ at time $t_{1}$, we derive a "mixed" correlation function. Note that the mixed correlation function is not simply a property of the fluid alone.

We shall make an assumption concerning the particle in relation to the fluid. This assumption, usully called the long wavelength hypothesis, states that the particle is small when compared with the scale of turbulence. To be more precise, we say that the (normalized) spatial 
correlation of fluid velorities is essentially unity over a region several partirle diameters wide about the particle. This assumption is necessary if we are to neglert viscous stress terms in the equations of motion. In chapter six, we will relax this assumption to state that the spatial rorrelation of fluid velorities is almost unity in a neighhorhood of the particle.

\subsection{The Diffusion Coeffirient}

The primary object of this thesis is the calculation of a diffusion coefficient for particles suspended in a turbulent fluid. Diffusion processes are usually calculated with a diffusion equation which can be derived as follows. Let $n$ be the number of particles per unit volume and let $F_{i}$ bet the flux of particles across a unit area. If $n$ were uniform in space, we would expect no net diffusion. Thus, the diffusion is intimately connected with the existence of spatial gradients of $\mathrm{n}$ and we write

$$
F_{i}=-D_{i j j} \frac{\partial n}{\partial x_{j}}
$$

where $D_{i j}$ is the (tensor) diffusion coefficient. We now emplny the Einstein convention of summation over repeated indires. This equation represents a macroscopic statement about the microscopir details of particle motion. Now consijer an arbitrary volume $V$ bnunded by the surface $\$$. The number of particles entering the volume as a function of time is the (negative) integral of the flux over the surface

$$
\frac{\partial}{\partial t} \int_{V} n d V=-\int_{\$} F_{i} \cdot d \phi_{i}
$$


where $d \$_{i}$ is the outward directed unit surface vectur. Commuting the time derivative and volume integration, and using the divergence theorem,

$$
\int_{V} \frac{\partial n}{\partial t} d V=-\int_{V} \frac{\partial F_{i}}{\partial x_{i}} d V=\int_{V} \frac{\partial}{\partial x_{j}} D_{i j} \frac{\partial n}{\partial x_{j}} d V
$$

This equation must hold for any volume, ience the intergrands must be equa 1

$$
\frac{\partial n}{\partial t}=\frac{\partial}{\partial x_{i}} D_{i j} \frac{\partial n}{\partial x_{j}}
$$

Because we have assumed that our fluid is statistically homogeneous, we can bring $D_{i j}$ outside the derivative and write

$$
\frac{\partial n}{\partial t}=D_{i j} \frac{\partial^{2} n}{\partial x_{i} \partial x_{j}}
$$

Now we need to relate our macroscopic picture of diffusion to the detailed microscopic description of particle motion. We do this in the following manner. We define a macroscopic average of a quantity $Q$ by

$$
\langle\mathrm{Q}\rangle=\int_{V} \mathrm{QndV} / \int_{V} \mathrm{ndV}
$$

We have already defined, the microscopir average as the ensemble average

$$
\bar{Q}=\text { ensemble average of } Q \text {. }
$$

We relate our two descriptions by assuming the equality of these two 
ave rages.

Let our particle begin at rest, at $x=0$ and $t=0$. Then in micro-. scopic picture, we have

$$
\begin{aligned}
z^{2}(t) & =\int_{0}^{t} d t 1 \int_{0}^{t} d t_{2} w\left(t_{1}\right) w\left(t_{2}\right) \\
& =\int_{0}^{t} d t 1 \int_{0}^{t} d t_{2} w\left(t_{1}\right) w\left(t_{2}\right)
\end{aligned}
$$

where 7 is the coordinate of a particle along the 3 -axis and $w$ is the particle velocity along the 3-axis.

In the macroscopic picture, we proceed as folluws. Multiply equation 1.2 by $z^{2}$ and integrate over all space. Here $z$ is the coordinate along the 3 -axis and differs from the quanitity $Z$.

$$
\int_{-(x)}^{(x)} z^{2} \frac{\partial n}{\partial t} d v=\int_{-(x)}^{x} D_{i j} z^{2} \underset{\partial x_{i}}{\dddot{x}^{2}-\frac{n}{\partial x_{j}}} d v
$$

where

$$
\int_{-\infty}^{(x)} d V \operatorname{means} \int_{-\infty x}^{(x)} d x \int_{-\infty)}^{\infty} d y \int_{-\infty)}^{\infty} d z .
$$

'The left-hand side may be written

$$
\frac{\partial}{\partial t} \int_{-\infty}^{\infty} \eta^{2} n d V
$$

for $z$ is just a courdinate and is independent of $t$ ime. The left hand side is then

$$
\frac{\hat{c}}{\partial t}\left\langle z^{2}(t)\right\rangle \int_{-\infty}^{\infty} n d V
$$


One term on the right-hand side of equation 1.3 is

$$
\int_{-\infty}^{\infty} D_{33} z^{2} \frac{\partial^{2} n}{\partial z^{2}} d V
$$

Doing the z-portion of the integral by parts twice, this becomes

$$
2 D_{33} \int_{-\infty}^{\infty} n d v
$$

winere we have assumed $\left(z^{2} \frac{\partial n}{\partial t}\right)_{-\infty}^{\infty}$ and $(z n)_{-c r}^{\infty}$ both vanish. This should be a good assumption, for diffusion equations typically lead to exponential decay in the spatial coordinates.

All the other terms on the right-hand side of equation 1.3

vanish if $\mathrm{n}$ is an even function. This must be the case if the initial distribution is even, for our fluid is statistically homogeneous and isotropic. A typical integral would be

$$
\begin{aligned}
\mathrm{D}_{23} \int_{-\infty}^{\infty} \mathrm{d} v \frac{\partial^{2} \mathrm{n}}{\partial \mathrm{y} \frac{\mathrm{z}}{\partial z} z^{2}} & =\mathrm{D}_{23} \int_{-\infty}^{\infty} \mathrm{dx} \int_{-\infty}^{\infty} \mathrm{dy} \int_{-\infty}^{\infty} \mathrm{d} z \frac{\partial^{2} \mathrm{n}}{\partial \mathrm{y} y} z^{2} \\
& =\mathrm{D}_{23} \int_{-\infty}^{\infty} \mathrm{dx} \int_{-\infty}^{\infty} \mathrm{d} z z^{2} \frac{\partial \mathrm{n}}{\partial z} \\
& =-2 \mathrm{D}_{23} \int_{-\infty}^{\infty} \mathrm{dx} \int_{-\infty}^{\infty} \mathrm{zndz}=0
\end{aligned}
$$

Equating the left and right sides,

$$
\frac{\partial}{\partial t}\left\langle Z^{2}(t)\right\rangle=2 D_{33}
$$

Now we connect the pictures by substituting $\overline{z^{2}(t)}$ for $\left\langle z^{2}(t)\right\rangle$. 
We find

$$
\left.2 D_{33}=\frac{\partial}{\partial t} \int_{0}^{t} d t_{1} \int_{0}^{t} d t_{2} \overline{w\left(t_{1}\right)} w_{(t}\right)
$$

or

$$
D_{33}=\int_{0}^{t} d t w_{1} \overline{w(t)}
$$

The same analysis holds for any component of $D_{i j}$ and we have, in general,

$$
D_{i j}=\frac{1}{2}\left[\int_{0}^{t} d t w_{i}\left(t_{1}\right) w_{j}(t)+\int_{0}^{t} d t \bar{w}_{i}(t) w_{j}\left(t_{1}\right)\right]
$$

where $w_{i}$ is the particle velocity along the $i$-axis.

Finally, we consider $D_{i j}$ in the limit of long times. If the time is long enough that the initial conditions are forgotten, then $w_{i}(t) w_{j}\left(t_{1}\right)$ becomes a stationary function $R_{i j}\left(t-t_{1}\right)$. We shall be able to show this and estimate the time required in chapter III. Since $\overline{w_{i}(t) w_{j}\left(t_{1}\right)}$ is a function which peaks at $t=t_{1}$, we have apapproximately

$$
\begin{aligned}
D_{i j} & =\lim _{t \rightarrow \infty} \int_{0}^{t} R_{i j}\left(t-t_{j}\right) d t_{1} \\
& =\lim _{t \rightarrow \infty} \int_{t}^{0} R_{i j}\left(t_{1}\right) d t_{1} \\
& =\lim _{t \rightarrow \infty} \int_{0}^{t} R_{i j}\left(t_{1}\right) d t_{1}
\end{aligned}
$$

or

$$
D_{i j}=\int_{0}^{\infty} R_{i j}\left(t_{1}\right) d t_{1}
$$




\section{CHAPTER II}

\subsection{Momentum Exchange in Two-Phase Flow}

In this chapter we will discuss the equations of motion of the particle. We begin by establishing rome conventions of notation. All quantities referring to the particle will be subscripted with a capitol " $P$ " and those referring to the fluid with a captol "F". Thus we have $\rho_{P}$, the microscopic particle density, and $\rho_{F}$, the microscopic fluid density. Total time derivatives will have an additional subscript which tells how to take the derivative. For example, $\left(\frac{d \vec{u}_{F}}{d t}\right)_{P}$ means the total time derivative of the fluid velocity along the motion of the particle. In operator notation

$$
\begin{aligned}
& \left(\frac{d}{d t}\right)_{P}=\left(\frac{\partial}{\partial t}+\vec{u}_{p} \cdot \nabla\right) \\
& \left(\frac{d}{d t}\right)_{F}=\left(\frac{\partial}{\partial t}+\vec{u}_{F} \cdot \nabla\right)
\end{aligned}
$$

We shall reserve the letter "v" for the relative velocity in this chapter.

$$
\vec{v} \equiv \vec{u}_{p}-\vec{u}_{F}
$$

The momentum equations for each component of a two-phase flow may be written (see, for example, Harlow and Amsden ${ }^{18}$ )

$$
\begin{aligned}
& \theta_{P} \rho_{P}\left[\frac{\partial \vec{u}_{P}}{\partial t}+\left(\vec{u}_{P} \cdot \nabla\right) \vec{u}_{P}\right]=-\theta_{P} \nabla \psi_{F}+\vec{v}_{P}+\theta_{P} \rho_{P} \vec{g}+\vec{F}_{e} \\
& \theta_{F} \rho_{F}\left[\frac{\partial u_{F}}{\partial t}+\left(\vec{u}_{F} \cdot \nabla\right) \vec{u}_{F}\right]=-\theta_{F} \nabla \psi_{F}+\vec{v}_{F}+\partial_{F} \rho_{F} \vec{g}-\vec{F}_{e}
\end{aligned}
$$


where

$$
\begin{aligned}
& \theta=\text { volume fraction } \\
& \vec{V}=\text { viscous force per unit volume } \\
& \psi_{F}=\text { pressure } \\
& \vec{F}_{t^{2}}=\text { momentum exchange force per unit volume } \\
& \vec{g}=\text { accleration due to gravity }
\end{aligned}
$$

In this equation, $\nabla \psi_{F}$ is the mean pressure gradient in the fluid independent of the presence of the particles. All details of the particle - fluid interaction are contained in $\vec{F}_{e}$. Note that we have assumed that the microscopic densities $\rho_{P}$ and $\rho_{F}$ are constant.

We picture our system as a very dilute cloud of particles suspended in the fluid. Hence we are concerned with equation 2.2 in the 1 imit

$$
\begin{aligned}
& \theta_{F} \rightarrow 1 \\
& \theta_{P}=1-\theta_{F}+0
\end{aligned}
$$

In this limit, we neglect $\vec{V}_{P}$ which is the viscous force of the particle field on itself. In the usual way, we set

$$
\overrightarrow{\mathrm{v}}_{F}=\mu_{F} \nabla^{2 \vec{u}_{F}}
$$

where $\mu_{F}=$ fluid viscosity. The quantity $\vec{F}_{\mathrm{e}}$ is the force of the fluid on a particle per unit volume multiplied by the product $\left(\theta_{P} \theta_{F}\right)$. In the limit $\theta_{P} \rightarrow 0$, 


$$
\begin{aligned}
& \overrightarrow{\mathrm{F}}_{\mathrm{e}} \rightarrow 0 \\
& \frac{\overrightarrow{\mathrm{F}}_{\mathrm{e}}}{\theta_{\mathrm{P}}} \rightarrow \frac{\overrightarrow{\mathrm{F}}}{4 \pi \mathrm{R}_{\mathrm{o}}^{3} / 3} \neq 0
\end{aligned}
$$

In words, the effect of the fluid on each particle is substantial, but the effect of a swall number of particles on the entire fluid is neg1igib1e.

Finally, we eliminate the pressure gradient between equations 2.2 . Using equation 2.1 , we find

$$
\rho_{P}\left(\frac{d \vec{u}_{P}}{d t}\right)_{P}=\rho_{F}\left(\frac{d \vec{u}_{F}}{d t}\right)_{F}-\mu_{F} \nabla^{2} \vec{u}_{F}+\left(\rho_{P}-\rho_{F}\right) \vec{g}+\vec{F} /\left(4 \pi R_{o}^{3} / 3\right)
$$

\subsection{The Force on a Particle}

We now turn vur at attention to $\vec{F}$, the force of the liquid on a particle. To find this force, we need to know the detailed fluid velocity distribution about the particle. At this point, we must carefully distinguish between two quaniities, both of which are called fluid velocities. The quantity $\vec{u}_{F}$ is the fluid velocity independent of the presence of the particle. It is the fluid velocity in equation 2.3 which appears as a result of elimination of the pressure gradient. We define a second fluid velocity $\vec{u}_{d}$ which incorporates the details of the fluid velocity due to the presence of the particle. This quantity is the one we use co calculate $\vec{F}$. The relationship between $\vec{u}_{F}$ and $\vec{u}_{d}$ is now described. 
The fluid velocity $\overrightarrow{u_{d}}$ is found by solving the time-dependent Navier-Stokes equation

$$
\begin{gathered}
\rho_{F}\left[\frac{\partial \vec{u}_{d}}{\partial t}+\left(\vec{u}_{d} \cdot \nabla\right) \vec{u}_{d}\right]=-\operatorname{grad} \psi+\mu_{F} \nabla^{2 \vec{u}_{d}} \\
\nabla \cdot \vec{u}_{d}=0
\end{gathered}
$$

with appropriate boundary conditions at the particle and at "inintity" and with initial conditions. Making use of the simplest long wavelength hypothesis (see chapter 1), we assume the fluid velocity far from the particle is some quantity $\vec{u}_{\infty}(t)$ which is uniform in space, though varying in time. The quantity $\vec{u}_{\infty}(t)$ is precisely the fluid velocity $\vec{u}_{F}(t)$. Thus, $\vec{u}_{d}$ is related to $\vec{u}_{F}$ though the boundary conditions applied to equation 2.4. We shall continue to use the notation $\vec{u}_{\infty}(t)$ in the following discussion of the force $\vec{F}$. Having the fluid velocity $\vec{u}_{d}$, we can calculate the stress tensor $\Sigma_{i j}$. The force, $f_{i}$, acting on a unit area of surface is

$$
\mathrm{f}_{i}=\Sigma_{i j} \eta_{j}=\left(\psi \delta_{i j}+\mu_{F}\left[\frac{\partial u_{d_{i}}}{\partial x_{j}}+\frac{\partial u_{d_{j}}}{\partial x_{i}}\right]\right) \eta_{j}
$$

where

$$
\begin{aligned}
& \psi=\text { pressure } \\
& \delta_{i j}=\text { Kronecker delta } \\
& \vec{n} \quad=\text { unit surface vector }
\end{aligned}
$$

and we are to sum over repeated indices. The total force on the particle 
then comes from integrating $f_{i}$ over the surface of the particle. In the expression for the force $f_{i}, \dot{\psi}$ is the pressure at the surface of the particle. This pressure is not an independent quantity, but is completely determined by the fluid velocity $\vec{v}_{d}$. In fact, the pressure obeys the Poisson equation

$$
\nabla^{2} \psi=\frac{\partial u_{d_{i}}}{\partial x_{j}} \frac{\partial u_{d_{j}}}{\partial x_{i}}
$$

Let IIs further assume, for the moment, that the particle Reynolds number

$$
\operatorname{Re}_{\mathrm{p}} \equiv \frac{|\mathrm{v}| \mathrm{R}_{\mathrm{O}}}{\nu} \ll 1
$$

where $\nu=\mu_{F} / \rho_{F}=$ kinematic viscosity. Note that this is a different quantity from the fluid Reynolds number which predicts the onset of turbulence in the fluid, and which depends, among other things, on the total fluid velocity $\vec{u}_{F}$. This assumption enables us to neglect the non-linear terms $\left(\vec{u}_{\mathrm{d}} \cdot \nabla\right) \vec{u}_{\mathrm{d}}$ (often called the convection terms) in the Navier-Stokes equation 2.4 .

The forces on a spherical particle, in the regime $\operatorname{Re}_{\mathrm{p}} \ll 1$ (called the stokes regime) are derived by Pearcey and $\mathrm{Hi}_{11}{ }^{20}$ and are found to be

$$
\begin{aligned}
& \vec{F}=-6 \pi \mu_{F} R_{0}\left(\vec{v}_{P}-u_{\infty}\right)-\frac{2 \pi}{3} R_{0}^{3} \rho_{F}\left(\frac{d \vec{u}_{P}}{d t}-\frac{d \vec{u}_{\infty}}{d t}\right)
\end{aligned}
$$

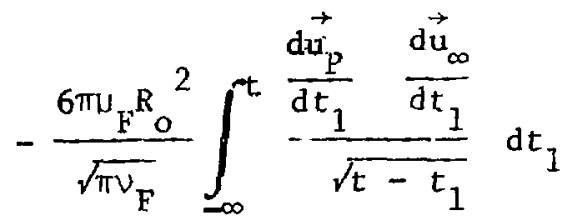


The first term is the Stokes force, a drag due to the relative velocity between fluid and particle. The second term is the virtual mass effect. The origin of the force lies in that when accelerating the particle, we must also accelerate the fluid around the particle. The net effect is to increase the apparent mass of the particle. The third term is the Basset history integral. Its origin lies in the fact that when the particle changes its state of motion, it takes a finite time for the fluid velocity to adjust jtself to its final steady-state distribution.

Let us now leave the Stokes regime and consider a flow regime in which the dominant turbulent frequencies are high and the amplitude of oscillations small. Calculation of the forces in this regime leads to exactly the same terms (Stokes plus Basset plus virtual n:ass) as in the Stokes regime. (See Landau and Lifshitz ${ }^{19}$, p. 97.)

The important unifying aspect of the two regimes is that we can neglect the convective terms of the Navier-Stokes equations when calculating the fluid velocity perturbed by the presence of the particle. For flows of large Reynolds numbers, when the convective terms cannot be neglected, a kind of force called form drag becomes important. This force depends quadratically on the relative velocity and is given by

$$
\vec{F}_{F}=C_{D} \pi \rho_{F}{ }_{o}^{2}\left(\vec{u}_{P}-\vec{u}_{\infty}\right)\left|\vec{u}_{P}-\vec{u}_{\infty}\right|
$$

(See Landau and Lifshitz ${ }^{18}, p .169$ ) where $C_{D}$ is a dimensionless constant of order one. The physical origin of this force lies in the separation of flow behind the particle. A comparison of Stokes drag and form drag shows the latter 
does not become important untii we reach Reynolds numbers of order ten where flow separation develops. Experimental evidence verifles this last prediction (see Landau and Lifshit ${ }^{19}, \mathrm{p}, 171$ ). In the regime of rapidiy oscillating flows, flow separation is precluded. On the basis of all these facts, we are led to speculate that the force law

$$
F=(\text { Stokes })+(\text { Basset })+(\text { Virtual Mass })
$$

is valid so long as there is no signiflcant flow separation.

Finally, we recall that in this derivation of the force on the particle due to the fluid, we have neglected the possibility of spatial gradient of $u_{F}$. Making this same approximation in equation 2.3 for the terms resulting from replacements of the pressure gradient, We derive the equation of motion for the particle

$$
\begin{aligned}
\frac{4 \pi R_{0}^{3}}{3} \rho_{P} \frac{d \vec{u}_{p}}{d t} & =\frac{4 \pi R_{0}^{3}}{3} \rho_{F} \frac{d \vec{u}_{F}}{d t}+\left(\rho_{P}-\rho_{F}\right) \frac{4 \pi R_{O}^{3}}{3} \vec{g} \\
& -a^{\prime}\left(\vec{u}_{P}-\vec{u}_{F}\right)-\frac{2 \pi R_{o}^{3}}{3}\left(\frac{d \vec{u}_{p}}{d t}-\frac{d \vec{u}_{F}}{d t}\right) \\
& -\kappa^{\prime} \int_{-\infty}^{t \frac{d \overrightarrow{1}_{P}}{d t}-\frac{d}{d t_{F}}} \frac{\sqrt{t-t_{1}}}{d t} t_{1}
\end{aligned}
$$

where $\alpha^{\prime}=6 \pi \mu_{F} R_{0}$

$$
\kappa^{\prime}=6 \pi \mu_{F} R_{0}^{2} / \sqrt{\pi v}
$$


In this approximation, we do not distinguish between the operators $\left(\frac{d}{d t}\right)_{P}$ and $\left(\frac{d}{d t}\right)_{F}$. Equation $2.5 \mathrm{can}$ be rewritten

$$
\begin{aligned}
\frac{d \vec{u}_{P}}{d t} & =\gamma \frac{d \vec{u}_{F}}{d t}+\frac{2\left(\rho_{P}-\rho_{F}\right)}{2} \frac{\rho_{F}+\rho_{F}}{g}-\alpha\left(\vec{u}_{P}-\vec{u}_{F}\right) \\
& -k \int_{-\infty}^{t\left[\frac{d \vec{u}_{P}}{d t}-\frac{d \vec{u}_{F}}{d t}\right]} \frac{\vec{l}_{1}}{\sqrt{t-t_{1}}} d t{ }_{1}
\end{aligned}
$$

where now

$$
\begin{aligned}
& \gamma=\frac{3 \rho_{F}}{2 \rho_{P}+\rho_{F}} \\
& \alpha=\frac{9 \mu_{F}}{R_{O}^{2}\left(2 \rho_{P}+\rho_{F}\right)} \\
& \kappa=\frac{9 \mu_{F}}{R_{0} \sqrt{\pi v}\left(2 \rho_{P}+\rho_{F}\right)}
\end{aligned}
$$

Equation 2.6 will form the basis of our work in chapters III and IV. The next higher order equation of motion comes from relaxing the long wavelength hypothesis in the following manner. Rather than assigning a uniform velocity

$$
\vec{u}_{\infty b}=\vec{u}_{F}
$$

we allow for the presence of spatial gradients

$$
\vec{u}_{\infty}=\vec{u}_{F}+\overrightarrow{\vec{\sigma}} \cdot \vec{x}
$$


in the derivation of the force laws. Here $\overrightarrow{\vec{\sigma}}$ is the tensor $\overrightarrow{\nabla u}_{F}$ and $\vec{x}$ is a vector whose origin is the center of the particle. To the same order of approximation, we shall have to distinguish between $\left(\frac{d}{d t}\right)$ and $\left(\frac{d}{d t}\right) F$. Derivation of the force laws in this approximation is not a trivial task and will be postponed to Chapter $V$.

\subsection{App 1fcations to Bubbles and Droplets}

We conclude this chapter by discussing the extension of the force laws to bubbles and droplets. So far, we have assumed that the particle is rigid. Inwever, Pearcey and Hill 20 also discuss the case where $\mu_{\mathrm{p}}$, the internal viscosity of the particle, is not infintte. In the low Reynolds number regime, assuming the particle remains spherical, Pearcey and Hill show that the equation of motion 2.7 remains the same, excepting only that the constants $\alpha$ and $\kappa$ must be redefined. In particular

$$
\alpha=\left(\frac{3 \mu_{P}+2 \mu_{F}}{3 \mu_{P}+3 \mu_{F}}\right)\left(\frac{9 \mu_{F}}{R_{0}^{2}\left(2 \rho_{P}+\rho_{F}\right)}\right)
$$

and

$$
k=\left(\frac{3 \mu_{P}+2 \mu_{F}}{3 \mu_{P}+3 \mu_{F}}\right)\left(\frac{9 \mu_{F}}{R_{o} \sqrt{\pi v} 2\left(\rho_{P}+\rho_{F}\right)}\right)
$$




\section{CHAPTER III}

3.1 Method of Rice Applied to the Simplified Equations of Motion In this chapter. we will calculate the diffusion coefficient for the particle using a simplified version of the equations of motion 2.5. Our purpose is twofold. First, we will illustrate our procedure for calculating the particle velocity correlation function and the diffusion coefficient. Second, we will compare our solution with previous work.

Let us simplify our notation by writing $\vec{w}=\vec{u}_{p}$ for the particle velocity and $\vec{u}=\vec{u}_{F}$ for the fluid velocity. Also, we will write $S$ meaning any diagonal element of the particle velocity correlation tensor and R meaning any diagonal element of the fluid velocity correlation along che motion of a particle. If we begin with equation 2.5 and neglect the Basset history term and the (gravitational) buoyancy term, we have

$$
\frac{d \vec{w}}{d t}=\gamma \frac{d \vec{u}}{d t}-\alpha x(\vec{w}-\vec{u})
$$

Our method proceeds as follows. We can write a formal solution L.f equation 3.1 .

$$
\begin{aligned}
\vec{w}(t) & =\gamma \vec{u}(t)+\exp -\alpha t[\vec{w}(0)-\vec{u}(0)] \\
& -\alpha(\gamma-1) \int_{0}^{t} \vec{u}\left(t^{\prime}\right) \exp \alpha\left(t^{\prime}-t\right) d t^{\prime} .
\end{aligned}
$$

From this equation, we can easily construct the particle velocity correlation. We can then calculate the diffusion coefficient $D(t)$ 
using equation 1.6 or 1.7 .

We will do this, but first we note that there is another, very elegant method of obtaining the particle velocity correlations. By analogy with a technique used in Brownian motion studies, we call this the method of Rice. The following derivation follows Levich. 7 To apply the method of Rice, we must make a few additional assumptions and definitions. We shall assume that after sufficient time has passed, the strchastic process $\vec{w}(t)$ becomes stationary in time. We shall assume that both $\vec{w}(t)$ and $\vec{u}(t)$ are ergodic in the correlation - i.e., that

$$
\begin{aligned}
S\left(t_{0}, t_{0}+t\right) & =\overline{w_{i}\left(t_{0}\right) w_{i}\left(t_{0}+t\right)} \\
& =\lim _{T \rightarrow \infty} \frac{1}{T} \int_{0}^{T} w_{i}\left(t_{0}\right) w_{i}\left(t_{0}+t\right) d t_{0}
\end{aligned}
$$

(no summation over the index i). For a stationary stochastic process, we define a quantity called the power spectrum as the Fourier transform of the correlation function

$$
P_{p}=\frac{1}{\sqrt{2 \pi}} \int_{-\infty}^{\infty} S(t) \exp -i \omega t d t
$$

and

$$
P_{F}=\frac{1}{\sqrt{2 \pi}} \int_{-\infty}^{\infty} R(t) \exp -i \omega t d t
$$


Then, if we define $A_{P}((1))$ and $A_{F}(1)$ as the Fourier transforms of $w(t)$ and $u(t)$ respectively, we have a very powerful theorem (see, For example, thlenbeck ${ }^{21}$ ) which states $\mathrm{P}_{\mathrm{p}}(\omega)$ is directly proportional to $\left|A_{P}(w)\right|^{2}$ and $P_{F}(w)$ is directly proportional to $\left|A_{F}(w)\right|^{2}$ with the same constant of proportionality.

Fourier transformirg equation 3.1 for one component of velocity, we have

$$
\begin{aligned}
& \left.\qquad \operatorname{i\omega A}_{p}(\omega)+\alpha A_{p}(\omega)=i \gamma(\omega) A_{p}(\omega)+\alpha A_{F}(\omega)\right) \\
& \text { so that }\left|A_{p}(\omega)\right|^{2}=\left|A_{F}(\omega)\right|^{2}\left|\frac{\alpha+i \gamma(\omega)}{\alpha+i(s)}\right|^{2}
\end{aligned}
$$

or

$$
P_{P}((i))=P_{F}(u)\left[\frac{\alpha^{2}+x^{2} \alpha^{2}}{\alpha^{2}+\frac{\alpha^{2}}{2}}\right]
$$

Now the particle correlation function is simply the inverse Fourier transforn of $\mathrm{P}_{\mathrm{p}}((1)$

$$
\begin{aligned}
& S(t)=\frac{1}{\sqrt{2 \pi}} \int_{-\infty}^{(x)} p_{p}(x) \exp i\left(s t d^{\prime} x\right) \\
& =-\frac{1}{\sqrt{2} \pi} \int_{-\infty}^{(\alpha)} P_{F}(\omega)\left[\frac{\alpha^{2}+\gamma^{2} \omega^{2}}{\alpha^{2}+\omega^{2}}\right] \exp i(\omega) d \omega \\
& =-\frac{Y^{2}}{\sqrt{2 \pi}} \int_{-\infty}^{\infty} P_{F}(u) \exp i(\omega) \mathrm{d} u \\
& +\frac{\left(1-\gamma^{2}\right)}{\sqrt{2 \pi}} \int_{-\infty}^{\omega} P_{F}(\omega)\left[\frac{\alpha^{2}}{\alpha^{2}+\omega^{2}}\right] \exp 1 \omega t d \omega .
\end{aligned}
$$




$$
S(t)=r^{2} R(t)+\frac{\alpha^{2}\left(1-x^{2}\right)}{\sqrt{2 \pi}} \int_{-\infty}^{(r)} \frac{P F(i) \exp i \text { unt } d(\alpha)}{\alpha^{2}+\omega^{2}}
$$

We prove the following 1emma. Let $f(t)$ and $g(t) t=$ the Fourier transforms of $F(\omega)$ and $G(\omega)$ respectively. Then

$$
\begin{aligned}
& \int_{-\infty}^{\infty} F(\omega) G(\omega) \exp i(\omega) t d \omega \\
& =\int_{-\infty}^{\infty} F(\omega) \frac{1}{\sqrt{2 \pi}} \int_{-\infty}^{\infty} g\left(t^{\prime}\right) \exp i \omega\left(t-t^{\prime}\right) d t^{\prime} d(t) \\
& =\int_{-\infty}^{\infty} g\left(t^{\prime}\right) \frac{1}{\sqrt{2 \pi}} \int_{-\infty}^{\infty} F(\omega) \exp i \omega\left(t-t^{\prime}\right) d \omega d t^{\prime} \\
& =\int_{-\infty}^{\infty} g\left(t^{\prime}\right) f\left(t-t^{\prime}\right) d t^{\prime}
\end{aligned}
$$

If we identify $F(\alpha)=\frac{1}{\alpha^{2}+\omega^{2}} ; G(\omega)=P_{F}(\omega)$; then

$$
\begin{aligned}
& g(t)=R(t) \\
& f(t)=\frac{1}{\sqrt{2 \pi}} \int_{-\infty}^{\infty} \frac{\exp i \omega t}{\alpha^{2}+\omega^{2}} d \omega=\sqrt{2 \pi} \frac{\exp -\alpha|t|}{2 \alpha} .
\end{aligned}
$$

Substituting into equation 3.3 , we have

$$
S(t)=\gamma^{2} R(t)+\left(1-\gamma^{2}\right) \frac{\alpha}{2} \int_{-\infty}^{\infty} R\left(t^{\prime}\right) \exp -\alpha\left|t-t^{\prime}\right| d t^{\prime}
$$

which be written 


$$
\begin{aligned}
S(t) & =\gamma^{2} R(t)+\frac{\alpha}{2}\left(1-\gamma^{2}\right) \int_{-x)}^{t} R\left(t^{\prime}\right) \exp \alpha\left(t^{\prime}-t\right) d t^{\prime} \\
& +\frac{\alpha}{2}\left(1-\gamma^{2}\right) \int_{t}^{(x)} R\left(t^{\prime}\right) \exp \left(x\left(t-t^{\prime}\right) d t^{\prime}\right.
\end{aligned}
$$

This is the expression for the partirle velocity correlation using " the method of Rice.

\subsection{The birect Integral Method}

At this point, we return to equation 3.2 to work the same troblem In another manner. Continuing the analogy with Brownian motion, Wo rall. our technique the nethod of direct integration. This method onjoys several advantages over the method of Rice. One which will soon become apparent is that we nerd not restrict ourselves to steady :state problems, but can take into account the effect of initial conditions. A second, more important advantage will appear in the ne::t chapter. It is possible tr apply this method to more general equations of motion and to solve problems previous atuthors have called intractable.

We proceed a!: follows. From equation 3.2, cualuated separately at times $t_{1}$ and $t_{2}$, we can write down $w\left(t_{1}\right)$ and $\dot{w}\left(t_{2}\right)$. For any component of these, we can multiply the two equations together and ensemble average. Interclianging the order of time integration and ensemble averaging, and using the fact that $\vec{w}(0)$ is uncorrelated with the flujd velocity at any time, we find 


$$
\begin{aligned}
& s\left(t_{1}, t_{2}\right)=\left[\overline{w_{i}{ }^{2}(0)}+\overline{\gamma^{2} u_{1}^{2}(0)}\right] \exp -\alpha\left(t_{1}+t_{2}\right) \\
& +\gamma^{2}\left[\overline{u_{i}\left(t_{1}\right) u_{i}\left(t_{2}\right)}-\overline{u_{i}\left(t_{1}\right) \overline{u_{i}(0)}} \exp -\alpha t_{2}\right. \\
& \left.-\overline{u_{i}(0) u_{i}\left(t_{2}\right)} \exp -\alpha t_{1}\right] \\
& -\alpha \gamma(\gamma-1)\left[\int_{0}^{t_{1}} \overline{u_{i}\left(t^{\prime}\right) u_{i}\left(t_{2}\right)} \exp \alpha\left(t^{\prime}-t_{1}\right) d t^{\prime}\right. \\
& \left.+\int_{0}^{t_{2}} \frac{}{u_{i}\left(t^{\prime}\right) u_{i}\left(t_{1}\right)} \exp \alpha\left(t^{\prime}-t_{2}\right) d t^{\prime}\right] \\
& +\alpha \gamma(\gamma-1) \exp -\alpha\left(t_{1}+t_{2}\right)\left[\int_{0}^{t} \frac{1}{u_{i}\left(t^{\prime}\right) u_{i}(0)} d t^{\prime}\right. \\
& \left.+\int_{0}^{t_{2}} \overline{u_{i}(0) u_{i}\left(t^{\prime}\right)} d t^{\prime}\right] \\
& +\alpha^{2}(\gamma-1)^{2} \int_{0}^{t} \int_{0}^{t} \frac{2}{u_{i}\left(t^{\prime}\right) u_{i}\left(t^{\prime \prime}\right)} \exp \alpha\left(t^{\prime}+t^{\prime \prime}-t_{1}-t_{2}\right) d t^{\prime} d t^{\prime \prime}
\end{aligned}
$$

(No summation over $i$. )

We emphasize once more that in this expression, $u_{i}(t)$ is the fluid velocity measured along the path of the particle. We also note that, with reference to Chapter II, $u_{i}(t)$ is the fluid velocity $\vec{u}_{F}$, not $\vec{u}_{d}$. Thus, $\overline{u_{i}\left(t_{1}\right) u_{i}\left(t_{2}\right)}$ is precisely what we have called $R\left(t_{1}-t_{2}\right)$

Thus, we can write equation 3.5 as

$$
\begin{aligned}
S\left(t_{1}, t_{2}\right) & =\left[S(0,0)+\gamma^{2} R(0)\right] \exp -\alpha\left(t_{1}+t_{2}\right) \\
& +\gamma^{2}\left[R\left(t_{2}-t_{1}\right)-R\left(t_{1}\right) \exp -\alpha\left(t_{2}\right)-R\left(t_{2}\right) \exp -\alpha t_{1}\right]
\end{aligned}
$$




$$
\begin{aligned}
& -\alpha Y(Y-1)\left[\int_{0}^{t} R\left(t^{\prime}-t_{2}\right) \exp \alpha\left(t^{\prime}-t_{1}\right) d t^{\prime}\right. \\
& \left.+\int_{0}^{t} R\left(t^{\prime}-t_{1}\right) \exp \left(t^{\prime}-t_{2}\right) d t^{\prime}\right] \\
& +\alpha Y(Y-1) \exp -\alpha\left(t_{1}+t_{2}\right)\left[\int_{0}^{t^{\prime}} R\left(t^{\prime}\right) d t^{\prime}\right. \\
& \left.+\int_{0}^{t_{2}} R\left(t^{\prime}\right) d t^{\prime}\right] \\
& +\alpha^{2}(Y-1)^{2} \int_{0}^{t} \int_{0}^{t^{2}} R\left(t^{\prime}-t^{\prime \prime}\right) \exp \alpha\left(t^{\prime}+t^{\prime \prime}-t_{1}-t_{2}\right) d t^{\prime} d t^{\prime \prime}
\end{aligned}
$$

Now we can simplify this expression. For example,

$$
\begin{aligned}
& \int_{0}^{t_{1}} R\left(t_{2}-t^{\prime}\right) \exp \alpha t^{\prime} d t^{\prime}=\exp \alpha t_{2} \int_{-t_{2}}^{t_{1}-t_{2}} R(\psi) \exp \alpha \psi d \psi \\
& \int_{0}^{t_{2}} R\left(t,-t^{\prime}\right) \exp \left(t^{\prime} d t^{\prime}=\exp \left(t_{1} \int_{-t_{1}}^{t_{2}-t_{1}} R(\psi) \exp \alpha \psi d \psi\right.\right.
\end{aligned}
$$

We also make use of a technique we shall frequently employ for reduction of the double integral term in equation 3.6 . We define a new set of variables

$$
\begin{aligned}
& \xi=\frac{\left(t^{\prime}-t^{\prime \prime}\right)}{\sqrt{2}} \quad t^{\prime}=\frac{\xi+n}{\sqrt{2}} \\
& n=\frac{\left(t^{\prime}+t^{\prime \prime}\right)}{\sqrt{2}} \quad t^{\prime \prime}=\frac{\eta-\xi}{\sqrt{2}}
\end{aligned}
$$


$-44-$

$$
\frac{\partial(\varepsilon, n)}{\partial\left(t^{\prime}, \frac{n}{\left.t^{\prime \prime}\right)}\right.}=1
$$

This is a rotation of the axes by $45^{\circ}$. We can write down the new limits of integration from the following diagram

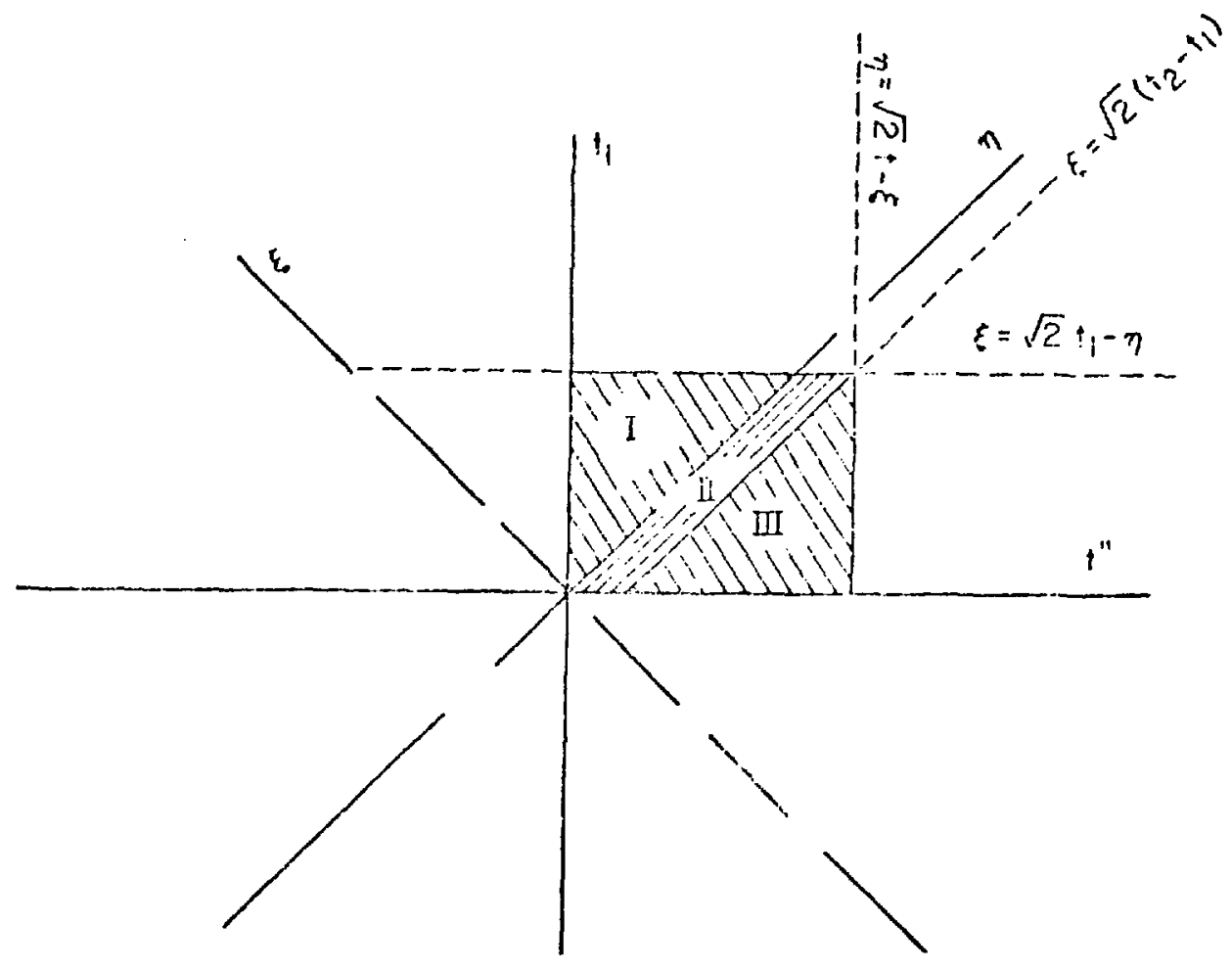

Then the double integral

$$
\begin{aligned}
I & =\int_{0}^{t} 1 d t^{\prime} \int_{0}^{t} 2 d t^{\prime \prime} R\left(t^{\prime}-t^{\prime \prime}\right) \exp \alpha\left(t^{\prime}+t^{\prime \prime}\right) \\
& =\int_{0}^{t} 1^{/ \sqrt{2}} R(\sqrt{2} \xi) d \xi \int_{0}^{\sqrt{2} t_{1}-\xi} \exp \alpha \sqrt{2} \eta d \eta
\end{aligned}
$$




$$
\begin{aligned}
& +\int_{\frac{t_{1}-t_{2}}{\sqrt{2}}}^{o} R(\sqrt{2} \xi) d \xi \int_{-\xi}^{\sqrt{2} t_{1}-\xi_{2}} \exp \alpha \sqrt{2} r d n \\
& +\int_{-t_{2} / \sqrt{2}}^{\frac{t_{1}-t_{2}}{\sqrt{2}}} R(\sqrt{2} \xi) d \xi \int_{-\xi}^{\xi+\sqrt{2} t_{2}} \exp \alpha \sqrt{2} n d \eta
\end{aligned}
$$

We want to establish. another convection of notation. In expressions like the one above, in which the right-hand side is the sum of several terms, we will refer to the separate terms as $I_{1}, I_{2}$, etc. In the order that they are uritten. If in addition, $I_{1}$ is found to be the sum of several terms, we will refer to them separately as $\mathrm{J}_{1}, \mathrm{~J}_{2}$, etc. Similarly, we will write $\mathrm{I}_{2}=\mathrm{K}_{1}+\mathrm{K}_{2}+\ldots$ and so on.

In each of the terms of equation 3.8, we can integrate over $n$. Setting $\xi=\sqrt{2} \xi$

$$
\begin{aligned}
& I_{1}=\frac{1}{2 \alpha} \int_{0}^{t_{1}} R(\psi)\left[\exp \alpha\left(2 t_{1}-\psi\right)-\exp \alpha \psi\right] d \psi \\
& I_{2}=\frac{1}{2 \alpha} \int_{t_{1}-t_{2}}^{0} R(\psi)\left[\exp \alpha\left(2 t_{1}-\psi\right)-\exp -\alpha \psi\right] d \psi \\
& I_{3}=\frac{1}{2 \alpha} \int_{-t_{2}}^{t_{1}-t_{2}} R(\psi)\left[\exp \alpha\left(2 t_{2}+\psi\right)-\exp -\alpha \psi\right] d \psi
\end{aligned}
$$

Thus, substituting equations 3.7 and 3.8 into 3.6 , we have 


$$
\begin{aligned}
& s\left(t_{1}, t_{2}\right)=\left[s(0,0)+\gamma^{2} R(0)\right] \exp -\alpha\left(t_{1}+t_{2}\right) \\
& +y^{2}\left[R\left(t_{2}-t_{1}\right)-R\left(t_{1}\right) \exp -\alpha t_{2}-R\left(t_{2}\right) \exp -\alpha t_{1}\right] \\
& +\frac{\alpha\left(1-\gamma^{2}\right)}{2}\left[\exp \alpha\left(t_{2}-t_{1}\right) \int_{t_{2}}^{t_{1}-t_{2}} R(\psi) \exp \alpha \psi d \psi\right. \\
& \left.+\exp \alpha\left(t_{1}-t_{2}\right) \int_{-t_{1}}^{t_{2}-t_{1}} R(\psi) \exp \alpha \psi d \psi\right] \\
& +\alpha \gamma(\gamma-1), \exp -\alpha\left(t_{1}+t_{2}\right)\left[\int_{0}^{t_{1}} R(\psi) d \psi+\int_{0}^{t_{2}} R(\psi) d \psi\right] \\
& -\frac{\alpha(\gamma-1)^{2}}{2} \exp -\left(t_{1}+t_{2}\right)\left[\int_{0}^{t_{1}} R(\psi) \exp \alpha \psi d \psi\right. \\
& \left.+\int_{0}^{t_{2}} R(\psi) \exp \alpha \psi d \psi\right]
\end{aligned}
$$

Note that the initial conditions appear in this expression and that the particle correlation is not stationary. Vote also that its correlation is symmetric in $t_{1}$ and $t_{2}$. To regain the expression we derived using the method of Rice, we must let $t_{1}+\infty$ and $t_{2} \rightarrow \infty$ in such a way that $t_{2}-t_{I}=t$ remains finite. The only surviving terms are

$$
\begin{aligned}
S\left(t_{1}, t_{2}\right)+S(t) & =\gamma^{2} R(t)+\frac{\alpha}{2}\left(1-\gamma^{2}\right) \exp -\alpha t \int_{-\infty}^{t} R(\psi) \exp \alpha \psi d \psi \\
& +\frac{\alpha}{2}\left(1-\gamma^{2}\right) \exp \alpha t \int_{-\left(x_{1}\right.}^{-t} R(\psi) \exp \alpha \psi d \psi
\end{aligned}
$$




$$
\begin{aligned}
S(t) \rightarrow \gamma^{2} R(t) & +\frac{\alpha}{2}\left(1-\gamma^{2}\right) \exp -\alpha t \int_{-(\alpha)}^{t} R(\psi) \exp \alpha \psi d \psi \\
& +\frac{\alpha}{2}\left(1-\gamma^{2}\right) \exp \alpha t \int_{t}^{\infty} R(\psi) \exp -\alpha \psi d \psi
\end{aligned}
$$

Thus, we regain equation 3.4 .

From this discussion, it becomes clear that the initial conditions are forgotten (and hence the particle velocity correlation becomes stationary) for times $t_{1}, t_{2} \gg \frac{1}{\alpha}$. Consider, for example, spherical carbon particles of radius $125 \cdot 10^{-4} \mathrm{~cm}$ and density $2.25 \mathrm{~g} \mathrm{~cm}{ }^{-3}$. For such particles, suspended in turbulent air, the particle response time is calculated to be

$$
\begin{aligned}
t_{R} & =\frac{1}{\alpha}=\frac{\mathrm{R}_{\mathrm{O}}^{2}\left(2 \rho_{\mathrm{D}}+\rho_{\mathrm{F}}\right)}{9 \mu_{\mathrm{F}}} \\
& =.52 \mathrm{sec}
\end{aligned}
$$

and the condition that initial conditions are forgotten becomes

$$
t>.52 \mathrm{sec} \text {. }
$$

On the time scales of air pollution problems, the correlation is almost instantaneously stationary. However, for these same carbon particles, if they wore used in the experiment of Snyder and Lumley ${ }^{27}$ where the particles are tracked for times like $.5 \mathrm{sec}$, the initial conditions of injection of the particles into the flow would become very important.

Finally, we want to calculate the diffusion coefficient. Because $S\left(t_{1}, t_{2}\right)=S\left(t_{2}, t_{1}\right)$, we can writ equation 1.6 as

$$
D(t)=\int_{0}^{t} s\left(t_{1}, t\right) d t_{1} .
$$


Substituting for $S$ from equation 3.9

$$
\begin{aligned}
& D(t)=\left[S(0,0)+\gamma^{2} R(0)\right] \int_{0}^{t} \exp -(x(t)+t) d t \\
& -\gamma^{2}\left[\int_{0}^{t} k\left(t-t_{1}\right)\right] \\
& -\gamma^{2}\left[\int_{0}^{t} R\left(t_{1}\right) \exp -\alpha t_{1} d t_{1}\right] \\
& -\gamma^{2}\left[\int_{0}^{t} R(t) \exp -\alpha t t_{1} d t\right] \\
& +\frac{\left(1-y^{2}\right)}{2}\left[\int_{0}^{t} \operatorname{cxp} \alpha\left(t-t_{1}\right) \int_{-t}^{t} 1^{-t} R(\psi) \operatorname{cxp}\right) \\
& +\frac{\alpha\left(1-\gamma^{2}\right)}{2}\left[\int_{0}^{t} \exp \alpha\left(t_{1}-t\right) \int_{-1: 1}^{t-1} 1 / x(\psi) \operatorname{xpc} \alpha \psi d \psi\right] \\
& \left.+\alpha \gamma(\gamma-1)\left[\int_{0}^{t} \exp -\alpha\left(t_{1}+t_{2}\right) \int_{0}^{t} r(n) d r\right)\right] \\
& +\alpha y(\gamma-1)\left[\int_{0}^{t} \exp -\alpha\left(t_{1}+t_{2}\right) \int_{0}^{l} l i(\psi) d \psi\right] \\
& +\frac{\alpha(\dot{\gamma}-1)^{2}}{2}\left[\int_{0}^{t} \exp -\alpha\left(t_{1}+t_{2}\right) \int_{0}^{l} 1 \quad l\left(l_{1}\right) \exp \alpha \psi c^{1} \psi\right] \\
& +\frac{\alpha(\gamma-1)^{2}}{2}\left[\int_{0}^{t} \exp -\alpha\left(t_{1}+t_{2}\right) \int_{0}^{t} R(\psi) \exp \alpha \psi d \psi\right]
\end{aligned}
$$

There are ten terms here, most of which can be simplified. For example,

$$
I_{1}=\left[S(0,0)+\gamma^{2} R(0)\right] \quad \int_{0}^{t} \exp -\alpha\left(t+t_{1}\right) d t t_{1}
$$


$-49-$

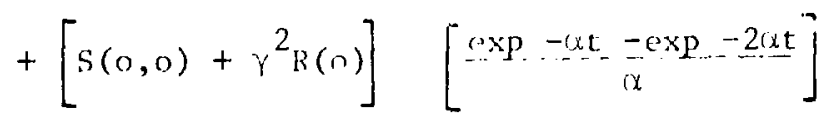

$$
\begin{aligned}
& I_{2}=r^{2} \cdot \int_{0}^{t} R\left(t-t_{1}\right) d t \\
& =\gamma^{2} \int_{0}^{t} R(\psi) d \psi \\
& I_{3}=-\gamma^{2} \exp -\alpha t \int_{0}^{t} R(\psi) d \cdot ! \\
& I_{4}=-\gamma^{2} R(t) \int_{0}^{t} \exp -\left(x t_{1} d t\right. \\
& =-\gamma^{2} R(t)\left[\begin{array}{ccc}
1 & -e x p & -\alpha t \\
& \cdots & x
\end{array}\right] \\
& T_{5}=\alpha\left(1-\frac{\left.-\gamma^{2}\right)}{2}\left[\int_{0}^{t} \exp i x\left(t_{1}-t\right) d t \int_{-t_{1}}^{t-t_{1}} R(\psi) \operatorname{cxp}\left(x \psi^{\prime} d \psi\right]\right.\right.
\end{aligned}
$$

Is ing the following diagram to invert the order of integration

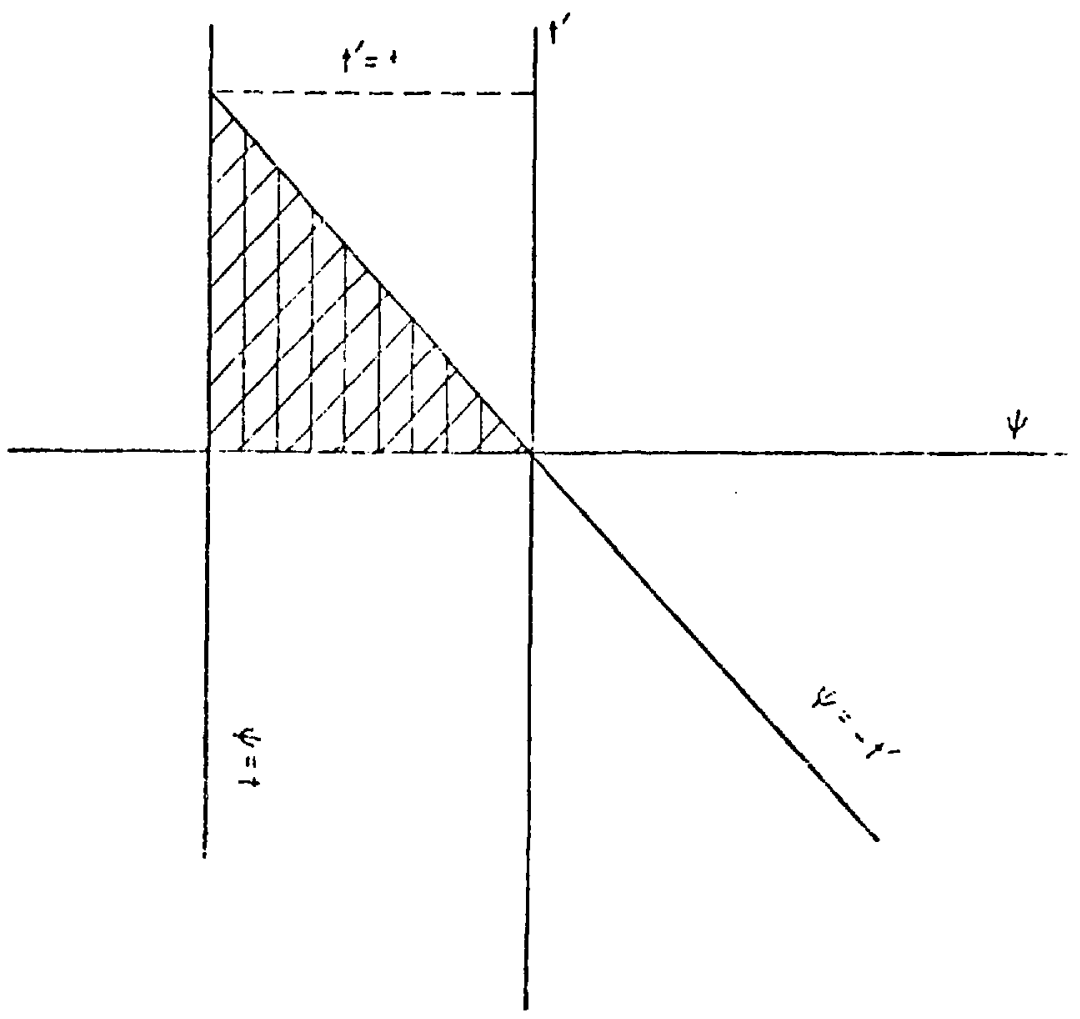




$$
\begin{aligned}
I_{5} & =\frac{\alpha\left(1-\gamma^{2}\right)}{2}\left[\int_{-t}^{0} d \psi R(\psi) \exp \alpha \psi \int_{-1,}^{t} \exp \alpha\left(t_{1}-t\right) d t_{1}\right. \\
& \left.+\int_{0}^{t} d \psi R(\psi) \exp \int_{0}^{t-\psi} \exp \alpha\left(t_{1}-t\right) d t_{1}\right] \\
& =\left(\frac{1-\gamma^{2}}{2}\right) \int_{0}^{t} d \psi R(\psi)[1-\exp \alpha(\psi-t)-\exp -\alpha t+\exp -\alpha \psi] \\
I_{6} & =\frac{\alpha\left(1-\gamma^{2}\right)}{2} \int_{0}^{t} \exp \alpha\left(t-t_{1}\right) d t_{1} \int_{-t}^{t_{1}-t} R(\psi) \exp \alpha \psi d \psi .
\end{aligned}
$$

Letting $t^{\prime}=t-t_{1}$

$$
I_{6}=\frac{\left(1-\gamma^{2}\right)}{2} \int_{0}^{t} \exp \alpha t^{\prime} d t^{\prime} \int_{-t}^{-t^{\prime}} R(\psi) \exp \alpha \psi d \psi
$$

We again invert the order of integration with the following diagram

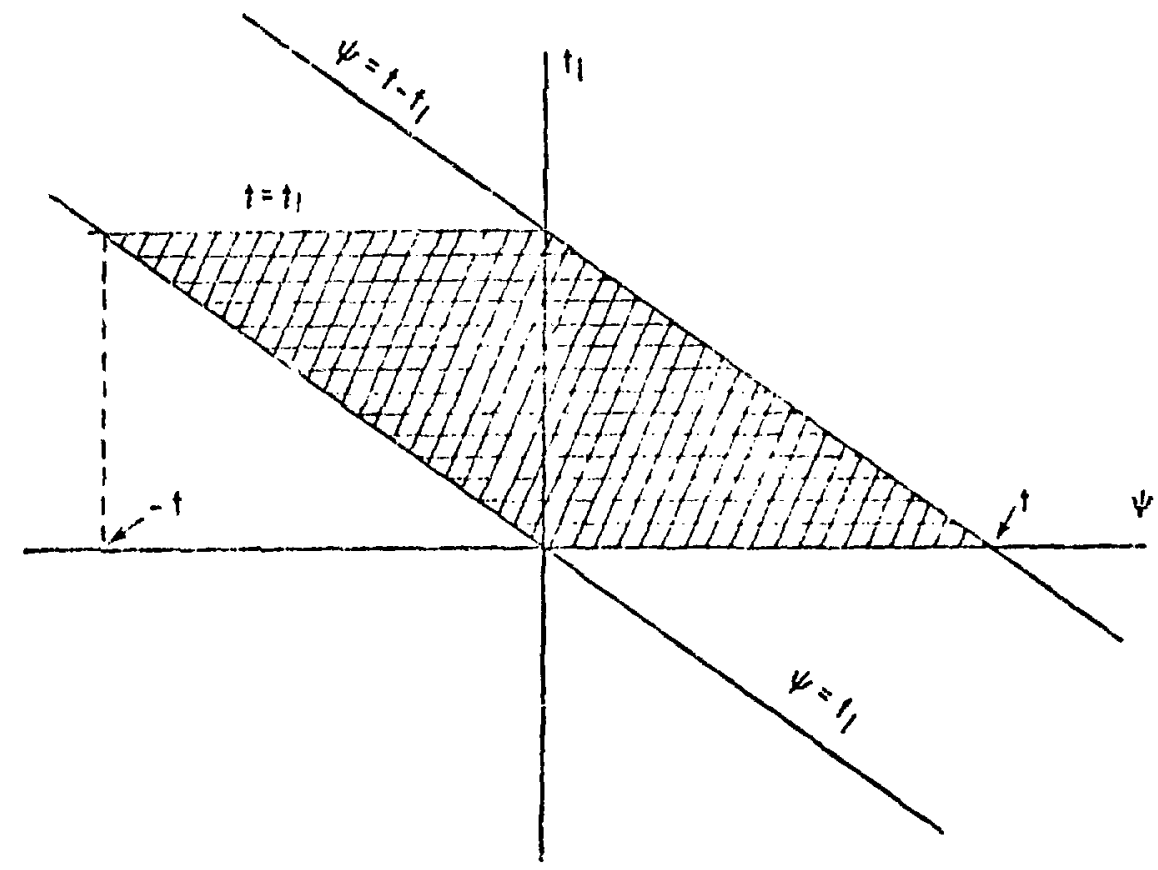




$$
\begin{aligned}
I_{6} & =\frac{\alpha\left(1-\gamma^{2}\right)}{2} \int_{-1}^{0} d \psi R(\psi) \exp x \psi^{\prime} \int_{0}^{-\psi} d t^{\prime} \exp d t^{\prime} \\
& =\left(1-\gamma^{2}\right) \int_{0}^{t} \mathrm{~d} \psi R(\psi)(1-\operatorname{rxp}-(x \psi) .
\end{aligned}
$$

Continuing, we have

$$
I_{7}=x y(r-1) \int_{0}^{t} \exp -\left(x\left(t_{1}+t_{2}\right) \int_{0}^{t} R(\psi) d \psi .\right.
$$

Inverting the order of integration with the following diagram

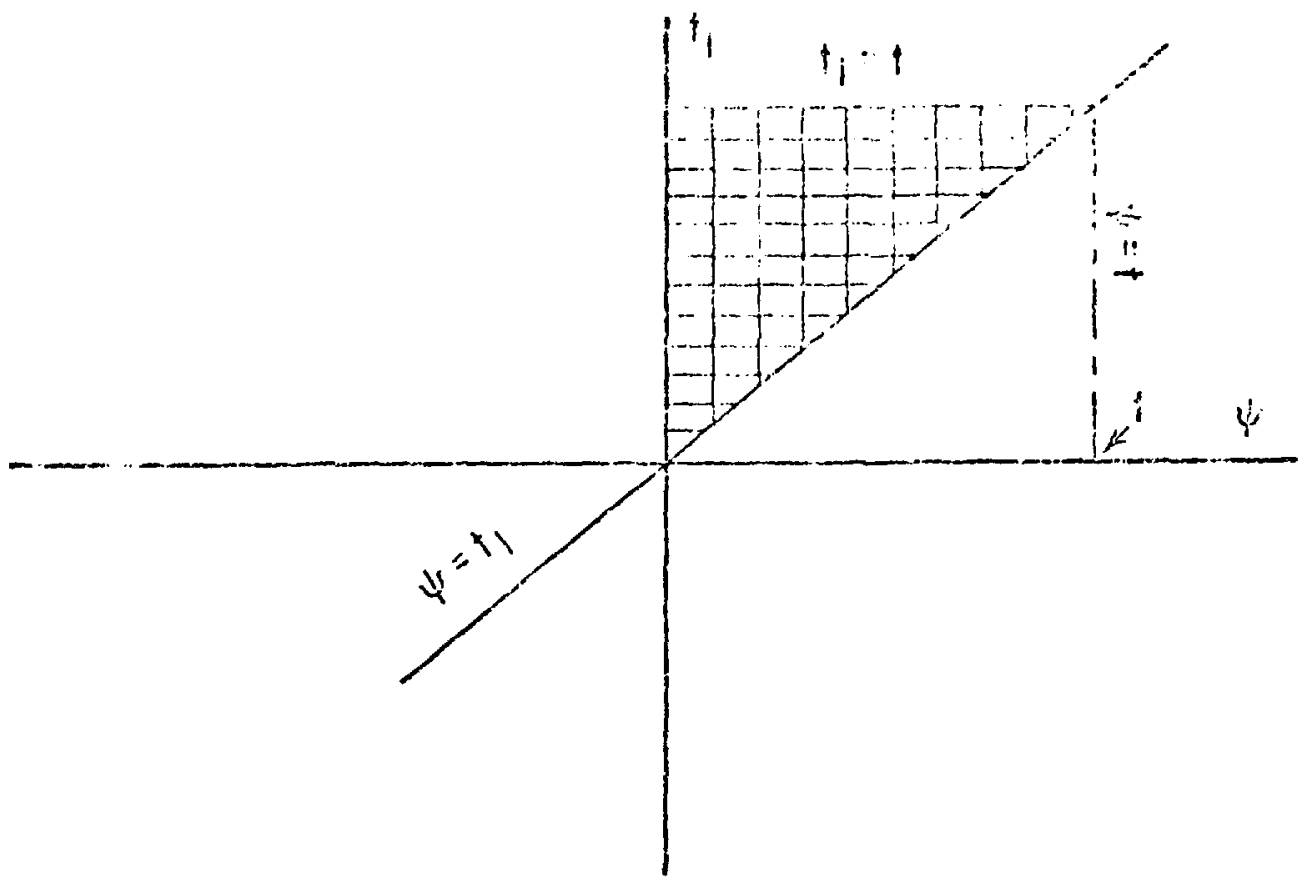

$$
\begin{aligned}
I_{7} & =\operatorname{cx} \gamma(\gamma-1) \int_{0}^{t} d \psi R(\psi) \int_{\psi}^{t} \exp -\alpha\left(t_{1}+t\right) d t{ }_{1} \\
& =\gamma(\gamma-1) \int_{0}^{t} d \psi r(\psi)[\exp -\alpha(t+\psi)-\exp -2 \alpha t]
\end{aligned}
$$


For

$$
\begin{aligned}
I_{8} & =\alpha \gamma(\gamma-1) \int_{0}^{t} \exp -\alpha\left(t_{1}+t\right) d t \int_{0}^{t} R(\psi) d \psi \\
& =\gamma(\gamma-2)[\exp -\alpha t-\exp -2 \alpha t] \int_{0}^{t} R(\psi) d \psi .
\end{aligned}
$$

We can use figure 3.4 again to invert the order of integration in I.9

$$
\begin{aligned}
1_{9} & =-\frac{\alpha(\gamma-1)^{2}}{2} \int^{t} \exp -\alpha\left(t+t_{1}\right) d t \int_{0}^{t} R(\psi) \exp \alpha \psi d \psi \\
& =-\frac{\alpha\left(\gamma-\frac{1}{2}\right)^{2}}{2} \int_{0}^{t} d \psi R(\psi) \exp \alpha \psi \int_{\psi}^{t} \exp -\alpha\left(t_{1}+t\right) d t_{1} \\
& =\frac{(y-1)^{2}}{2} \int_{0}^{t} d \psi R(\psi)[\exp \alpha(\psi-2 t)-\exp -\alpha t]
\end{aligned}
$$

And finally

$$
\begin{aligned}
I_{10} & =-\frac{\alpha(\gamma-1)^{2}}{2} \cdot \int_{0}^{t} \exp -\alpha\left(t+t_{1}\right) d t \int_{0}^{t} R(\psi) \exp \alpha \psi d \psi \\
& =\frac{(\gamma-1)^{2}}{2} \int_{0}^{t} d \psi R(\psi)[\exp \alpha(\psi-2 t)-\exp \alpha(\psi-t)] .
\end{aligned}
$$

Now we can substitute al1 these results into equation 3.11 to derive

$$
\begin{aligned}
D(t) & =\left[S(0,0)+\gamma^{2} R(0)\right]\left[\frac{\exp -\alpha t-\exp -2 \alpha t}{\alpha}\right] \\
& -\gamma(\gamma-1) \exp -\alpha t \int_{0}^{t} R(\psi) d \psi \\
& -\gamma^{2} R(t)\left[\frac{1-\exp -\alpha t}{\alpha}\right] \\
& +\gamma \exp -\alpha t \int_{0,}^{t} R(\psi) \exp u \psi d \psi
\end{aligned}
$$




$$
\begin{aligned}
& +(\gamma-1)^{2} \exp -2 \alpha t \int_{0}^{t} R(\psi) \exp \alpha \psi d \psi \\
& +2 \gamma(\gamma-1) \exp -2 \alpha t \int_{0}^{t} R(\psi) d \psi \\
& +\gamma(\gamma-1) \exp -\alpha t \int_{0}^{t} R(\psi) \exp -\alpha \psi \\
& +\int_{0}^{t} R(\psi) d \psi
\end{aligned}
$$

In this limit of long times (by which we mean $t \gg \frac{1}{\alpha}$ ), we have

$$
\begin{aligned}
\lim _{t \rightarrow \infty} D(t) & =\alpha \lim _{t \rightarrow \infty} \exp -\alpha t \int_{0}^{t} R(\psi) \exp \alpha \psi d \psi \\
& +\int_{0}^{x_{1}} R(\psi) d \psi
\end{aligned}
$$

where we have used the facts

$$
\lim _{t \rightarrow \infty} R(t)=0
$$

and

$$
\int_{0}^{\infty} R(\psi) d \psi<\infty .
$$

This last follows from the fact that the integral is bounded by the passive scalar diffusion coefficient of the fluid which we assume is finite. A simple application of L'Hôpital's rule shows

$$
\begin{aligned}
\lim _{t \rightarrow \infty} \exp -\alpha t \int_{0}^{t} \exp \alpha \psi R(\psi) d \psi \\
\quad=\lim _{t \rightarrow \infty} \frac{\exp \alpha t \operatorname{R}(t)}{\alpha \exp \alpha t}=0 .
\end{aligned}
$$


So we find that, for long time

$$
D(t) \rightarrow \int_{0}^{\infty} R(\psi) d \psi
$$

We conclude this chapter by discussing this last result.

Let us assume, with Tchen $^{4}$, that the fluid velocity correlation $R(t)$ is the same function as the Lagrangian correlation of fluid velocities. Then equation 3.13 says

$$
\lim _{t \rightarrow \infty} D(t)=D_{F}
$$

where $D_{F}$ is the passive scalar diffusion coefficient. In this limit, the particle diffusivity is independent of the particle density and radius. But suppose the partirle has a very large density compared to fluid density. Then one would expect the particle to respond very sluggishly to the fluid, and its diffusivity to be much less than passive scalar diffusivity. If the particle could have infinite density, it would not move at a 11 and its diffusivity would be zero.

There are two points to consicier in this regard. First, equation 3.14 is not really independent of particle density and radius in the sense that the limit of "long times" is defined in terms of these quantities. In particular, we have found that

$$
t \rightarrow \infty \text { means } t>\frac{1}{\alpha}
$$

or

$$
t \gg \frac{R_{o}^{2}\left(2 \rho_{p}+\rho_{F}\right)}{9 \mu_{F}}
$$


If the particle could truly have infinite density, there would be no time sufficiently large that equation 3.14 would apply.

Second, we must qucstion whether Tchen's assumption, concerning the equivalence of $R(t)$ and the Lagrangian correlation function, is valid in the case of very dense partirles. Let us attempt to answer this question on the basis of the following crude model. First, we suppose that the function $R(t)$ can be represented more accurately as the Lagrangian correlation reduced by the spatial correlation corresponding to the "distance" between the particle and its original neighborhood of fluid. This assumption is justified for certain rases where the joint distrjbution of fluid velocities at separale points is nearly Gaussian (see Peskin"). Further, we approximate this "distance" as

$$
\left|\int v\left(t^{\prime}\right) d t\right| \sim \sqrt{v^{2} t}
$$

where $v$ is the relative velocity between particle and fluid in one dimension. This should be a good estimate for times on the order of, and much smaller than the correlation time $r_{L}$. For times much greater than $t_{L}$, the Lagrangian correlation exp $-\left|t / t_{L}\right|$ is nearly zero, and it should not matter how we estimate the "distance". Now let us assume some specific forms for the correlation functions, For the Lagrangian correlation, we will use

$$
\text { Lagrangian correlation }=\exp -\left|t / t_{L}\right|
$$

This formi agrees with experimental data and is used by many authors 
(see Hinze ${ }^{4}$, p. 4h6, Levich ${ }^{7}$, Snyder and Lumley ${ }^{15}$ ). For the spatial correlation, we use

$$
\text { spatial correlation }=\exp -\left|x / \ell_{c}\right|
$$

This form is also consistent with experimental dala (see Hinze ${ }^{4}$, p.187, Snyder and Lumley ${ }^{27}$, Then

$$
R(t)-u^{2} \quad \exp -\left|\frac{t}{t}\right|-\left|\sqrt{\frac{v^{2}}{t}} \frac{u_{c}}{e^{2}}\right|
$$

It is also useful to employ the approximate relation

$$
\ell_{c} \sim t_{L} j \overline{u^{2}}
$$

(Csanady ${ }^{27}$, p.54, Snyder and Lumley ${ }^{15}$ ), to write

$$
R(t) \quad \overline{u^{2}} \quad \exp -\left|\frac{t}{t}\right|\left(1+\sqrt{\frac{v^{2}}{u^{2}}}\right)
$$

In this form, we see that $R(t)$ is we11 approximated by the Lagrangian correlation whenever the mean square relative velocity is much less than the mean square fluctuating fluid velority. We can estimate $v^{-\overrightarrow{2}}$ in the following manner. By definition

$$
\begin{aligned}
\overline{v^{2}} & =\overline{(w-u)^{2}} \\
& =\overline{w^{2}}+\overline{u^{2}}-\overline{2 u w}
\end{aligned}
$$

Now $\overline{w^{2}}$, in the limit of long time, can be found from equation 3.4 by 
setting $t=0$.

$$
\begin{aligned}
\overline{w^{2}} & =\gamma^{2} R(0)+\frac{\alpha}{2}\left(1-\gamma^{2}\right) \int_{-\infty}^{o} R\left(t^{\prime}\right) \exp \alpha t^{\prime} d t^{\prime} \\
& +\frac{\alpha}{2}\left(1-\gamma^{2}\right) \int_{0}^{\infty} R\left(t^{\prime}\right) \exp -\alpha t^{\prime} d t^{\prime}
\end{aligned}
$$

To lowest order, we vrite

$$
R(t)=\overline{u^{2}} \exp -\left|\frac{t}{t_{L}}\right|
$$

and

$$
\overline{w^{2}}=\gamma^{2} \overline{u^{2}}+\overline{u^{2}}\left(1-\gamma^{2}\right) \frac{a t_{L}}{\alpha t_{L}+1}
$$

Also, in the limit of long times

$$
w(t)=\gamma u(t)-\alpha(\gamma-1) \int_{0}^{t} u\left(t^{\prime}\right) \exp \alpha\left(t^{\prime}-t\right) d t^{\prime}
$$

(equation 3.2 where we have ignored the initial conditions) and so

$$
\begin{aligned}
\overline{w(t) u(t)} & =\gamma R(0)-\alpha(\gamma-1) \int_{0}^{t} R\left(t-t^{\prime}\right) \exp \alpha\left(t^{\prime}-t\right) d t^{\prime} \\
& =\gamma \overline{u^{2}}-(\gamma-1) \overline{u^{2}} \frac{\alpha t_{L}}{\alpha t_{L}+1}
\end{aligned}
$$

Finally, then 


$$
\begin{aligned}
\overline{v^{2}} & =(\gamma-1)^{2} \overline{u^{2}}-(\gamma-1)^{2} \overline{u^{2}} \frac{\alpha t_{L}}{\alpha t_{L}+1} \\
& =u^{2} \frac{(\gamma-1)^{2}}{\alpha t_{L}+1}
\end{aligned}
$$

or

$$
\frac{\overline{v^{2}}}{u^{2}}=(\gamma-1)^{2} \frac{1}{\alpha t_{L}+1}
$$

or

$$
\left.\frac{\overline{v^{2}}}{u^{2}}=\left(\frac{2 \rho_{F}-2 \rho_{p}}{2 \rho_{p}+\rho_{F}}\right)^{2} \frac{1}{\left(\frac{9 v_{F} t_{L}}{R_{o}^{2}\left(2 \rho_{p}+\rho_{F}\right)}+1\right.}\right)
$$

In the limit $\frac{\rho_{\mathrm{p}}}{\rho_{\mathrm{F}}} \rightarrow \infty, \frac{\overline{v^{2}}}{\mathrm{u}^{2}} \rightarrow 1$. This is reasonable, for when the particle becomes infinitely dense, it does not move at a11, and so the mean square relative velocity should equal the mean square fluctuating fluid velocity.

$$
\text { So, if } \frac{\rho_{p}}{\rho_{F}} \rightarrow \infty \text {, the correlation } R(t) \text { cannot be assumed equivalent }
$$
to the Lagrangian correlation and this is a second reason to explain the failure of equation 3.14 for very dense particles. One can also see that the Lagrangian correlation may be assumed equal to $R(t)$ when the particle density is very nearly equal to the fluid density, or when the particle response time $\left(\frac{1}{\alpha}\right)$ is much smaller than the correlation time $\left(t_{L}\right)$. 
Throughotul Chapter IIJ, we have neglected the Basset history term in the particle equation of motion. Hienfelt and Mockros 22 point out that this neglect is justified only when the frequencies which characterize the turbulence are very small. In the next chapter we will proceed to incorporate the Basset term in the particle equation of motion. 


\section{CHAPTER IV}

\subsection{Formal. Solution of the Equations of Motion}

In this chapter we will modify the the calculations of Chapter III by including the Basset term and the gravitational buoyancy term in the particle equation of motion. In the notation of Chapter III, we begin with

$$
\frac{d \vec{w}}{d t}=\gamma \frac{d \vec{u}}{d t}-\alpha(\vec{w}-\vec{u})-\kappa \int_{-\infty}^{t} \frac{1 \frac{d \vec{w}}{d t_{1}-\frac{d \vec{u}}{d t_{1}}}}{\sqrt{t-t_{1}}} d t_{1}
$$

$$
+\alpha \vec{c}
$$

where $\vec{G}=\frac{2\left(\rho_{p}-\rho_{F}\right)}{\alpha\left(2 \rho_{p}+\rho_{F}\right)} \vec{g}$

and $\mathrm{g}=-980 \mathrm{~cm} \mathrm{sec}^{-2}$ in the upward vertical direction.

Before beginning these calculations, we need to make the following comments to put the results of this chapter into proper perspective. Tchen ${ }^{3}$ first tackled the problem of including the Basset term in the particle equation of motion. It was Tchen who first showed how to transform equation 4.1 into an ordinary second-order differential equation. However, Tchen's analysis is not completely general. In particular, his method of calculating the particle diffusivity is not valid whenever $\rho_{p}<1.75 \rho_{F}$. Also, in this range of dealsities, his solution to the equation of motion is unstable except for the particular choice of initial conditions $\vec{w}=0$ and $\vec{u}=0$.

The new results of this chapter can be summarized as follows. 
First, we will derive an analytic solution to the dynamical problem described by equation 4.1. This solution will be valid for all times, and for arbitrary initial conditions, and for arbitrary particle density. Second, we will calculate a time-dependent diffusion coefficient for the particle. We will investigate the long-time limit and the early time limit for the particle diffusivity.

$$
\begin{aligned}
& \text { First, let us define a new variable } \\
& \vec{q}=\vec{w}-\vec{u}-\vec{G}
\end{aligned}
$$

In index notation, we rewrite equation 4.1

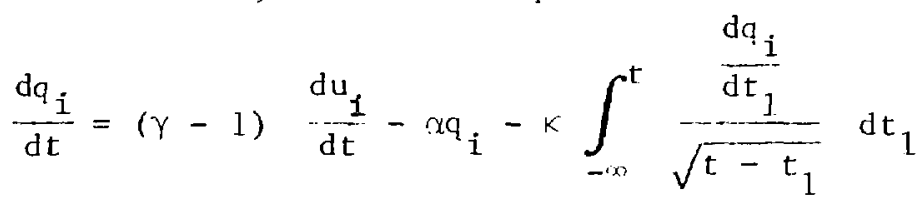

Notice that the integral in the last term extends in time from

$-\infty$ to $t$, independent of the starting time of the problem. Thus, if we wish to follow the motion of the particle beginning at some finite time (say $t=0$ ) we must supply both $q_{i}(0)$ and the entire history of $\frac{d q_{i}}{d t}$ from time $-\infty$ to 0 . The physical reason for this is intimately tied to the nature of the Basset force. The Basset force arises from the fact that when the particle is acrelerated, it takes a finite time for the fluid velocity to adjust itself. To specify the exact fluid velocity surrounding the particle at $t=0$, it is necessary to know the entire acceleration history of the particle for all previous times. The equation of motion of the particle for times greater than $t=0$ is 
$\frac{d q_{1}}{d t}=\alpha q_{i}-k \int_{0}^{t} \frac{\frac{d q_{i}}{d t}}{\sqrt{t-t_{1}}}+f_{i}(t)$

where

$$
q_{i}(0)=-u_{i}(0)-G_{i}+w_{i}(0)
$$

and

$$
f_{i}(t)=(\gamma-1) \frac{d u_{i}}{d t}-k \int_{-\infty}^{0} \frac{\frac{d q_{i}}{d t_{i}}}{\sqrt{t-t_{1}}} d t_{1} .
$$

We can transform equation 4.2 into an ordinary, second-order differential equation. Following Tchen, we first note

$$
I=\int_{t_{1}}^{t_{2}} \frac{d t}{\sqrt{\left(t_{2}-t\right)\left(t-t_{1}\right)}}=\pi \text {. }
$$

Now consider

$$
J=\int_{0}^{t} \frac{d t}{\sqrt{t_{2}-t}} \int_{0}^{t} \frac{\phi\left(t_{1}\right)}{\sqrt{t-t_{1}}} d t 1
$$

where $\phi$ is an arbitrary function. Inverting the order of integration, we have

$$
J=\int_{0}^{t_{2}} \phi\left(t_{1}\right) d t_{1} \int_{t_{1}}^{t_{2}} \frac{d t}{\sqrt{\left(t_{2}-t\right)\left(t-t_{1}\right)}}
$$

or

$$
J=\pi \int_{0}^{t_{2}} \phi\left(t_{1}\right) d t_{1}
$$


Now begin with equation 4.2 , multiply through by $\frac{1}{\sqrt{s}-\frac{t}{-t}}$ and integrate over $t$ between 0 and $s$. Ising equation 4.3, we derive

$$
\begin{aligned}
\int_{0}^{s} \frac{\frac{d q_{i}}{d t}}{s-t} d t=-x \int_{0}^{s} \frac{q_{i}}{\sqrt{s-t}-\pi k\left(q_{i}(s)-q_{i}(o)\right)} \\
+\int_{0}^{s} d t \frac{f_{i}(t)}{\sqrt{s-t}} .
\end{aligned}
$$

Again, multiply equation 4.4 through by $\frac{1}{\sqrt{n-i}}$ and integrate over s between 0 and $\%$. We find

$$
\begin{aligned}
q_{i}\left(r_{1}\right)-q_{i}(0) & =-\alpha \int_{0}^{n} q_{i}(s) d s-k \int_{0}^{n} \frac{q_{i}(s)}{\sqrt{\eta}-s} d s \\
& +k \int_{0}^{n} \frac{q_{i}(0)}{\sqrt{n}-s} d s+\int_{0}^{1} f_{i}(s) d s .
\end{aligned}
$$

Next, we define

$$
\begin{aligned}
& A_{i}=\int_{0}^{t} \frac{\frac{d q_{i}}{d t}}{\sqrt{t-n}} d n \\
& B_{i}=\int_{0}^{t} \frac{q_{i}(n)}{\sqrt{t}-n_{1}} d n .
\end{aligned}
$$

Equations $4.2,4.4$ and 4.5 constitute a system of three equations which can be written 


$$
\begin{aligned}
& \frac{d q_{i}}{d t}=-\alpha q_{i}-k A_{i}+f_{i} \\
& A_{i}=-\alpha B_{i}+\pi r\left(q_{i}(t)-q_{i}(0)\right)+\int_{0}^{t} d \eta \frac{f_{i}(n)}{\sqrt{t}-\eta} \\
& q_{i}(t)=q_{i}(0)-\alpha \int_{0}^{t} q_{i}(s) d s-k B_{i} \\
& \quad+\int_{0}^{t} f_{i}(n) d \eta+\int_{0}^{t} \frac{q_{i}(0)}{\sqrt{t}-n} d \eta .
\end{aligned}
$$

We algebraically eliminate $A_{i}$ and $B_{i}$ from this system. Differentiating the result once with respect to time, we finally derive

$$
\frac{d^{2} q_{j}}{d t^{2}}+\left(2 \alpha-\pi k^{2}\right) \frac{d q_{i}}{d t}+\alpha^{2} q_{i}=F_{i}(t)
$$

where

$$
\begin{aligned}
F_{i}(t) & =\frac{d}{d t}\left[f_{i}(t)-k \int_{0}^{t} d n \frac{f_{i}(n)}{\sqrt{t-n}}\right]+\alpha f_{i}(t) \\
& +k \alpha q_{i}(0) \frac{d}{d t}\left[\int_{0}^{t} \frac{d n}{\sqrt{t-n}}\right] .
\end{aligned}
$$

The formal solution of this equation is

$$
\begin{aligned}
q_{i}(t) & =\int_{b}^{t} F_{i}(s)\left[\frac{\exp r_{2}(t-s)-\exp r_{1}(t-s)}{r_{2}-r_{1}}\right] d s \\
& +a_{2} \exp r_{2} t+a_{1} \exp r_{1} t
\end{aligned}
$$


where

$$
\begin{aligned}
& r_{1}=\frac{\kappa^{2} \pi-2 \alpha}{2}-\frac{\sqrt{k^{2} \pi\left(k^{2} \pi-4 \alpha\right)}}{2} \\
& r_{2}=\frac{\kappa^{2} \pi-2 \alpha}{2}+\frac{\sqrt{k^{2} \pi\left(\kappa^{2} \pi-4 \alpha\right)}}{2}
\end{aligned}
$$

Substituting this solution into equation 4.2 , we find

$$
\begin{aligned}
& a_{1}=\frac{-f_{i}(o)}{r_{2}-r_{1}}+\frac{q_{i}(o) \kappa^{2} \pi \alpha}{\left(r_{2}-r_{1}\right)\left(r_{1}+\alpha\right)} \\
& a_{2}=\frac{-f_{i}(o)}{r_{2}-r_{1}}+\frac{q_{i}(o) k^{2} \pi \alpha}{\left(r_{2}-r_{1}\right)\left(r_{2}+\alpha\right)} .
\end{aligned}
$$

Integrating by parts the terms in $F_{i}$ (s) containing time derivatives, we can write a formal solution for the particle velocity

$$
\begin{aligned}
w_{i}(t) & =\int_{0}^{t} d s f_{i}(s)\left[\frac{\left(r_{2}+\alpha\right) \exp r_{2}(t-s)-\left(r_{1}+\alpha\right) \exp r_{1}(t-s)}{r_{2}-r_{1}}\right] \\
& -k \int_{0}^{t} d s \int_{0}^{t} d n \frac{f_{i}(r)}{\sqrt{s-n}}\left[\frac{r_{2} \exp r_{2}(t-s)-r_{1} \exp r_{1}(t-s)}{r_{2}-r_{1}}\right] \\
& +k \alpha q_{i}(0) \int_{0}^{t} d s\left[\frac{\exp r_{2}(t-s)-\exp r_{1}(t-s)}{\left(r_{2}-r_{1}\right) \sqrt{s}}\right] \\
& -\frac{k^{2} \pi q_{i}(0) \alpha}{r_{2}-r_{1}}\left[\frac{\exp r_{2} t}{r_{2}+(x}-\frac{\exp r_{1} t}{r_{1}+\alpha}\right]+u_{i}(t)+G_{i} \cdot
\end{aligned}
$$

where 
$f_{i}(t)=(\gamma-1) \frac{d u_{i}}{d t}-k \int_{-\infty}^{\infty} \frac{\frac{d q_{i}}{d t}}{\sqrt{t-t_{1}}} d t_{1}$

and

$$
\begin{aligned}
& r_{1}=\frac{\kappa^{2} \pi-2 \alpha}{2}-\frac{\sqrt{k^{2} \pi\left(\kappa^{2} \pi-4 \alpha\right)}}{2} \\
& r_{2}=\frac{\kappa^{2} \pi-2 \alpha}{2}+\frac{\sqrt{k^{2} \pi\left(\kappa^{2}\right.}}{2}-\frac{\pi-4 \alpha)}{}
\end{aligned}
$$

Let us pause at this point to consider the two parameters $r_{1}$ and $\mathrm{r}_{2}$. We define

$$
\beta=\frac{\alpha}{\kappa^{2} \pi}=\frac{2 \rho_{p}}{9 \rho_{F}}
$$

Thus we can write

$$
\begin{aligned}
& r_{1}=\kappa^{2} \pi\left(\frac{1-2 \beta}{2}-\frac{\sqrt{1-4 \beta}}{2}\right) \\
& r_{2}=\kappa^{2} \pi\left(\frac{1-2 \beta}{2}+\frac{\sqrt{1-4 \beta}}{2}\right) .
\end{aligned}
$$

clearly $\frac{1}{9}<\beta_{m} \leqslant \infty$. The case $\beta=\infty$ corresponds to $k=0$ which is the case we solved in Chapter III. The other possibilities are

Case 1: $\frac{1}{9}<8<\frac{1}{4}$.

Then $r_{1}$ and $r_{2}$ are both real and positive.

Case 2: $\quad \beta=\frac{1}{4}$.

Then $r_{1}$ and $r_{2}$ are both real, positive and equal. 
Case 3: $\frac{1}{4}<\beta<\frac{1}{2} \quad$.

Then $r_{1}$ and $r_{2}$ are complex conjugates with positive real part.

Case $4: \beta=\frac{1}{2}$.

Then $r_{1}$ and $r_{2}$ are complex conjugates whose real part is zero.

Case $5: \frac{1}{2}<\beta<\infty$.

Then $r_{1}$ and $r_{2}$ are complex conjugates whose real part is negative.

It seems strange at first sight that our solution might contain exponentials with positive real parts. It will turn out that these represent physical solutions. To see this, let us return to equation 4.8 and insert the detailed expression for $f_{i}(t)$. We assume now that the real parts of $r_{1}$ and $r_{2}$ are positive.

It is useful to note that $r_{1}$ and $r_{2}$ obey the algebraic identities $\alpha+r_{2}-k \sqrt{\pi} r_{2}^{3 / ;}=0$.

and

$$
\alpha+r_{1}-k \sqrt{\pi} r_{1}^{\frac{1}{2}}=0
$$

We also note

$$
\int_{0}^{\infty} \frac{\exp r(t-s)}{\sqrt{s}} d s=r^{-3} \sqrt{\pi} \exp r t
$$

if the real part of $r$ is positive. 
Now consider the third and fourth terms of equation 4.8 .

$$
\begin{aligned}
I_{3}+I_{4}=k \alpha q_{i}(0) & \int_{0}^{t} d s\left[\frac{\exp r_{2}(t-s)-\exp r_{1}(t-s)}{\left(r_{2}-r_{1}\right) \sqrt{s}}\right] \\
& -\frac{\kappa^{2} \pi q_{i}(0) \alpha}{r_{2}-r_{1}}\left[\frac{\exp r_{2} t}{r_{2}+\alpha} \frac{\exp r_{1} t}{r_{1}+\alpha}\right]
\end{aligned}
$$

We add and subtract $\operatorname{kaq}_{i}(0) \int_{t}^{\infty} \mathrm{ds}\left[\frac{\exp \mathrm{r}_{2}(t-s)-\exp \mathrm{r}_{1}(t-s)}{\left(\mathrm{r}_{2}-\mathrm{r}_{1}\right) \sqrt{s}}\right]$

$$
\begin{aligned}
I_{3}+I_{4} & =-k \alpha q_{i}(0) \int_{t}^{\infty} d s\left[\frac{\exp r_{2}(t-s)-\exp r_{1}(t-s)}{\left(r_{2}-r_{1}\right) \sqrt{s}}\right] \\
& +\frac{k \alpha q_{i}(0) \sqrt{\pi}}{r_{2}-r_{1}}\left[\frac{\exp r_{2} t}{r_{2}+\alpha}\left(r_{2}+\alpha-k \sqrt{\pi} r_{2}^{\frac{1}{2}}\right)\right] \\
& -\frac{k \alpha q_{i}(0) \sqrt{\pi}}{r_{2}-r_{1}}\left[\frac{\exp r_{1} t}{r_{1}+\alpha}\left(r_{1}+\alpha-k \sqrt{\pi} r_{1}^{\frac{1}{2}}\right)\right] \\
& =-k \alpha q_{i}(0) \int_{t}^{\infty}\left[\frac{\exp r_{2}(t-s)-\exp r_{1}(t-s)}{\left(r_{2}-r_{1}\right) \sqrt{s}}\right]
\end{aligned}
$$

We can manipulate the other terms of equation 4.8 in the same manner and, after some algebra, derive

$$
w_{i}(t)=\gamma u_{i}(t)+G_{i}
$$




$$
\begin{aligned}
& -k(\gamma-1) \int_{0}^{t} d \eta u_{i}(\eta) \int_{t}^{x:}\left[\frac{r_{2} \exp r_{2}(t-s)-r_{1} \exp r_{1}(t-s)}{2(s-\eta)^{3 / 2}\left(r_{2}-r_{1}\right)}\right] d s \\
& -k(\gamma-1) u_{i}(0) \int_{t}^{(x)} d s\left[\frac{r_{2} \exp r_{2}(t-s)-r_{1} \exp r_{1}(t-s)}{s^{\frac{1}{2}}\left(r_{2}-r_{1}\right)}\right] \\
& +k^{2} \int_{0}^{t} d n\left[\int_{-\infty}^{0} \frac{d u_{i}}{\sqrt{n}-t_{1}} d t_{1}\right] \\
& {\left[\int_{t}^{\infty} \frac{r_{2} \exp r_{2}(t-s)-r_{1} \exp r_{1}(t-s)}{\left(r_{2}-r_{1}\right)(s-n)}\right] d s} \\
& -\operatorname{krq}_{i}(0) \int_{t}^{\infty} d s\left[\frac{\exp r_{2}(t-s)-\exp r_{1}(t-s)}{\left(r_{2}-r_{1}\right) \sqrt{s}}\right]
\end{aligned}
$$

Now we propose the following problem. Suppose that previous to some time $t_{0}>0$, the fiuid and particle are at rest. At $t_{0}$, the fluid velocity suddenly becomes equal to a constant $U_{i}$ and remains at this value for all times after. Mathematically, we write

$$
u_{i}(t)=u_{i} H\left(t-t_{0}\right)
$$

when $H\left(t-t_{0}\right)$ is the Heaviside function. If we also ignore the gravitational term $G_{i}$, the equation of motion becomes, for $t>t_{0}$,

$$
w_{i}(t)=\gamma U_{i}-k(\gamma-1) U_{i} \int_{0}^{t} d n \int_{t}^{\infty}\left[\frac{r_{2} \exp r_{2}(t-s)-r_{1} \exp r_{1}(t-s)}{2(s-n)^{3 / 2}\left(r_{2}-r_{1}\right)}\right] d s
$$


liet $\xi=s-n$ in the inner integral and theis invert the order cf integration

$$
\begin{aligned}
& w_{i}(t)=\gamma U_{i}-k(\gamma-1) u_{i} \int_{0}^{t} d \xi \\
& \int_{t-\zeta,}^{t}\left[\frac{r_{2} \exp r_{2}(t-\xi-n)-r_{1} \exp r_{1}\left(t-\xi_{-}-n\right)}{2(\xi)^{3 / 2}\left(r_{2}-r_{1}\right)}\right] d n \\
& -k(\gamma-1) \int_{i} \int_{t}^{|x|} d \xi \int_{0}^{t} d n\left[\begin{array}{rr}
r_{2} \exp r_{2}(t-\xi,-n)-r_{1} \exp r_{1}\left(t-\xi_{,}-n\right) \\
2(\xi)^{3 / 2}\left(r_{2}-r_{1}\right)
\end{array}\right] \\
& =\gamma u_{i}+k(\gamma-1) u_{i} \int_{u}^{l} d \xi\left[\frac{\exp -r_{2} \xi-\exp -r_{1} \xi}{2\left(r_{2}-r_{1}\right) \xi^{3 / 2}}\right] \\
& +k(\gamma-1) u_{i} \int_{t}^{\infty} d \xi\left[\frac{\exp -r_{2} \xi_{2} \exp -r_{1} \xi_{0}}{2\left(r_{2}-r_{3}\right) \xi_{0}^{3 / 2}}\right] \\
& -k(\gamma-1) u_{i} \int_{t}^{\infty} \mathrm{d} \xi,\left[\frac{\exp r_{2}(t-\xi)-\exp r_{l}(t-\xi)}{2\left(r_{2}-r_{1}\right) \xi^{3 / 2}}\right]
\end{aligned}
$$

Now as $t \rightarrow \infty$, the 1 ast term vanishes and

$$
\begin{aligned}
\lim _{t \rightarrow \infty} w_{i}(t) & =\gamma u_{i}-k(\gamma-1) u_{i} \int_{0}^{\infty n} d \xi\left[\begin{array}{c}
\exp -r_{2}-\exp -r_{j} \xi \\
2\left(r_{2}-r_{1}\right) \xi^{3 / 2}
\end{array}\right] \\
& =\gamma U_{i}-k(\gamma-1) u_{i} \int_{0}^{\infty} d \xi\left[\frac{r_{2} \exp -r_{2} \xi_{j}-r_{1} \exp -r_{1} \xi}{\left(r_{2}-r_{1}\right) \xi^{3}}\right]
\end{aligned}
$$




$$
\begin{aligned}
& =\gamma u_{i}-k(\gamma-1) u_{i} \sqrt{\pi}\left(\begin{array}{c}
r_{2}-r_{1}{ }^{\prime} \\
r_{2}-r_{1}
\end{array}\right) \\
& =u_{i}
\end{aligned}
$$

This result says that the particle velocity remains bounded and tends to the physically correct limit of the fluid velocity for long times. Since equation 4.10 is linear, we san superpose an arbitrary number of Heaviside functions to reproduce any fluid velority distribution for $t: 0$.

In equation 4.9 , it is rlear that the fourth and sixth terms die away to zero as $t \rightarrow x$. If we write $q_{i}$ as a sum of Heaviside function for $t \leqslant 0$, we will find the fifth term also tends to zero as $t \rightarrow \infty$ for each Heaviside input. Thus, we conclude equation 4.9 (and hence also equation 4.8 ) is a well-behaved solution for the particle velocity for the cases of real part of $r_{1}$ and $r_{2}$ positive and for arbitrary initial conditions. Also, since equation 4.8 is we 11-behaved for the case of the real parts $r_{1}$ and $r_{2}$ negative, we conclude that this equation is a valid solution of particle velocity for all possible values of $B$.

\subsection{Comments on the Formal Solution}

In this section, we wish to make two comments on the formal solution 4.8. The first comment concerns the effect of gravity in our calculations. When we construct the diffusion coefficient, we will encounter terms involving the products $\overline{u_{i} G_{i}}$ and $\overline{G_{i} G_{i}}$, no 
summation over $i$ being implied. The first term vanishes, for $\overrightarrow{u_{i} G_{i}}=\overrightarrow{u_{i} G_{i}}=0$. The second term is, of course, just the constant $\left(G_{i}\right)^{2}$

Physically, a particle in a gravitational field moving in viscous fluid will attain a terminal velocity (equal to $G_{i}$ here). We are interested in the diffusion of the particle relative to this terminal velocity and, because $\overline{u_{i} G_{i}}$ vanishes, this is the same diffusivity as we would calculate in the absence of gravity. So, from this point on, we will set $G_{i}$ equal to zero in our calculations. To make the second comment, we must refer back to equation 4.8 . We have already concluded that this expression is valid for all values of $\beta$ in the interval $\frac{1}{9}<\beta<\infty$. Suppose we fix the value of $k^{2} \pi$ and rewrite equations 4.8 in terms of the parameter $\beta$. It is easy to see, by constructing the expansion of this expression in powers of $\beta$, that $w_{i}$ as given by equation 4.8 is an analytic function of $\beta$ everywhere in the interval $\frac{1}{9}<\beta<\infty$.

In deriving equation 4.9, we made the assumption $\frac{1}{9}<\beta<\frac{1}{2}$ and this equation can be seen to be analytic only for values of $\beta$ in this interval. Suppose now that we use equation 4.9 to calculate a particle diffusivity and further suppose this diffusivity is analytic over a connnected region larger than $\frac{1}{9}<\beta<\frac{1}{2}$. Then we can show that the derived diffusivity is the correct expression for any value of $B$ in the larger region of analyticity. The proof goes as follows. First we note that the product or sum of analytic functions is itself analytic. Thus, the diffusivity is an analytic function 
of the particle velocity. The particle ve $i t y$, as given by equation 4.8 , is, in turn, an analytic function of $\quad$ over the interval $\frac{1}{9}<B<\infty$. Knowing that an analytic function of an analytic function of $B$ is itself an analytic function of $\beta$, we conclude that paticle diffusivity can be written as an analytir funtion of $\quad \beta$ over the interval $\frac{1}{9}<\beta<\infty$.

Now suppose we derive from equation 4.9 an expression for particle diffusivity analytic over some range of $R$. Invoking the identity theorem for analytic functions, ${ }^{23}$ we may conclude that the expression is valid over any connected region in which it is analytic.

Equation 4.9 is a correct expression for the particle velocity in the interval $\frac{1}{9}<\beta<\frac{1}{2}$. It. is valjd for eny choice of initial conditions. We will make a sresific choice of initial conditions to simplify our calculations. Let us assume that the particle and fluid are at rest for all times $t<0$. At $t=0$, we imagine the fluid begins to move in some continuous, but otherwise arbitrary fashion. Setting $G_{i}$ to zero, we can write the particle volocity as

$$
w_{i}(t)=\gamma u_{i}(t)-k(\gamma-1) \int_{0}^{t} \int_{t}^{\infty} d s d r_{1}\left[\begin{array}{c}
r_{2} \exp r_{2}(t-s)-r_{1} \exp r_{1}(t-s) \\
2(s-\eta)^{3 / 2}\left(r_{2}-r_{1}\right)
\end{array}\right]
$$

4.3 Calculation of Partirle Diffusivity with Basset Forces Our first task is to construct the particle velority correlation $s\left(t, t_{1}\right)=\overline{w_{i}(t) w_{i}(t)}$ (no summation over $\left.i\right)$. It is convenient to rewrite equation 4.11 in the form 


$$
\begin{aligned}
w_{i}(t)= & \gamma u_{i}(t)-k(\gamma-l) \int_{0}^{t} d s u_{i}(t-s) \int_{0}^{u s} d \xi \\
& {\left[\frac{r_{2} \exp -r_{2} \xi-r_{1} \exp -r_{1} \xi_{0}}{2(\xi+s)^{3 / 2}\left(r_{2}-r_{1}\right)}\right] }
\end{aligned}
$$

Then we write

$$
\begin{aligned}
& S\left(t, t_{1}\right)=\gamma^{2} R\left(t-t_{1}\right) \\
& -k \gamma(\gamma-1) \int_{0}^{t} d s R\left(t_{1}-s-t\right) \int_{0}^{\prime \prime \prime} d \xi \bullet \\
& {\left[\frac{r_{2} \exp -r_{2} \xi-r_{1} \exp -r_{1} \varepsilon}{2(\xi+s)^{3 / 2}\left(r_{2}-r_{1}\right)}\right]} \\
& -k \gamma(\gamma-l) \int_{0}^{1} d s R(t+s-t) \int_{0}^{\prime \prime} d \zeta \\
& {\left[\frac{r_{2} \exp -r_{2} \xi-r_{1} \exp -r_{1} \xi}{2(\xi+s)^{3 / 2}\left(r_{2}-r_{1}\right)}\right]} \\
& +\kappa^{2}(\gamma-1)^{2} \int_{0}^{t} d s_{2} \int_{0}^{t} d s_{1} R\left(t-s_{2}-t+s_{1}\right) \int_{1}^{\infty} d \varepsilon_{1} \int_{1}^{\infty} d \xi_{2} \\
& {\left[\frac{r_{2} \exp -r_{2}^{\xi_{1}}-r_{1} \exp -r_{1} \xi_{2}}{2\left(\xi_{1}+s_{1}\right)^{3 / 2}\left(r_{2}-r_{1}\right)}\right]} \\
& {\left[\frac{r_{2} \exp -r_{2} \xi_{2}-r_{1} \exp -r_{1} \xi_{2}}{2\left(\xi_{2}+s_{2}\right)^{3 / 2}\left(r_{2}-r_{1}\right)}\right]}
\end{aligned}
$$


The diffusivity is then

$$
D(t)=\int_{0}^{t} d t_{1} s\left(t, t_{1}\right)
$$

or

$$
\begin{aligned}
& D(t)=\gamma^{2} \int_{0}^{t} d t_{]} \quad R\left(t-t_{1}\right) \\
& -k(\gamma-1) \gamma \int_{0}^{t} d t 1 \int_{0}^{t} d s R\left(t l^{-s-t)} \int_{0}^{\infty} d \xi\right. \\
& {\left[\frac{r_{2} \exp -r_{2} \xi-r_{1} \exp -r_{1} \xi}{2(\xi+s)^{3 / 2\left(r_{2}-r_{1}\right)}}\right]} \\
& +k(\gamma-1) \gamma \int_{0}^{t} d t \int_{0}^{t} d s R\left(r_{1}+s-t\right) \int_{0}^{\infty} d \xi
\end{aligned}
$$

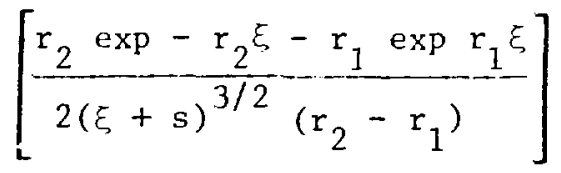

$$
\begin{aligned}
& +\kappa^{2}(\gamma-1)^{2} \int_{0}^{t} d t_{1} \int_{0}^{t} d s_{2} \int_{0}^{t} d s_{1} R\left(t-s_{2}-t_{1}+s_{1}\right) \int_{0}^{\infty} d \xi_{1} \int_{0}^{\infty} d \xi_{2} \\
& {\left[\frac{r_{2} \exp -r_{1} \xi_{1}-r_{1} \exp -r_{1} \xi_{1}}{2\left(r_{2}-r_{1}\right)\left(\xi_{1}+s_{1}\right) 3 / 2}\right]\left[\frac{r_{2} \exp -r_{2} \xi_{2}-r_{1} \exp -r_{1} \xi_{2}}{2\left(r_{2}-r_{1}\right)\left(\xi_{2}+s_{2}\right)^{3 / 2}}\right]}
\end{aligned}
$$

We can simplify the second term as follows 


$$
\begin{aligned}
I_{2} & =\int_{0}^{t} d t \int_{0}^{t_{1}} d s R(t-s-t) \frac{1}{2(\xi+s)^{3 / 2}} \\
& =\int_{0}^{t} d z \int_{0}^{t-z} d s R(: t+z) \frac{1}{2(\xi,+s)^{3 / 2}}
\end{aligned}
$$

Transform variables, letting

$$
\begin{array}{ll}
x_{1}=\frac{s+z}{\sqrt{z}} & s=\frac{x_{1}+x_{2}}{\sqrt{2}} \\
x_{2}=\frac{s-z}{\sqrt{2}} & z=\frac{x_{1}-x_{2}}{\sqrt{2}}
\end{array}
$$

Then $t / \sqrt{2}$

$$
\begin{aligned}
I_{2} & =\int_{0}^{t / \sqrt{2}} d x_{1} R\left(\sqrt{2} x_{1}\right) \int_{-x_{1}}^{x_{1}} d x_{2} \frac{1}{2\left(\xi+\frac{x_{1}+x_{2}}{\sqrt{2}}\right)^{3 / 2}} \\
& =\int_{0}^{t / \sqrt{2}} \sqrt{2} d x_{1} R\left(\sqrt{2} x_{1}\right)\left[\frac{1}{\left.\xi^{\frac{T}{2}}-\frac{1}{\left(\xi+\sqrt{2} x_{1}\right)^{\frac{r}{2}}}\right]}\right. \\
& =\int_{0}^{t} d x R(x)\left(\frac{1}{\xi^{\frac{1}{2}}}-\frac{1}{(\xi+x)^{\frac{1}{3}}}\right)
\end{aligned}
$$

The entire second term can then be written

$$
\begin{aligned}
I_{2} & =-k(\gamma-1) \gamma \int_{0}^{t} d x R(x)\left(\frac{r_{2}-r_{1}}{r_{2}-r_{1}}\right) \sqrt{\pi} \\
& +k(\gamma-1) \gamma \int_{0}^{t} d x R(x) \int_{0}^{\infty}\left[\frac{r_{2} \exp -r_{2} \xi-r_{1} \exp -r_{1} \xi}{(\xi+x)^{\frac{1}{s}}\left(r_{2}-r_{1}\right)}\right] d \xi
\end{aligned}
$$

I In similar fashion, the third term can be written 


$$
\begin{aligned}
& I_{3}=r(y-1) \gamma \int_{0}^{t} d x R(x)\left(\begin{array}{c}
r_{2}-r_{1} ! \\
\hdashline r_{2}-r_{1}
\end{array}\right) \sqrt{\pi} \\
& -k(\gamma-1) \gamma \int_{0}^{l} d x R(x) \int_{0}^{1}\left[\begin{array}{c}
r_{2} \exp -r_{2}-r_{1} \exp -r_{1} \xi_{2} \\
(\xi+x) \\
\left(r_{2}-r_{1}\right)
\end{array}\right] d \xi
\end{aligned}
$$

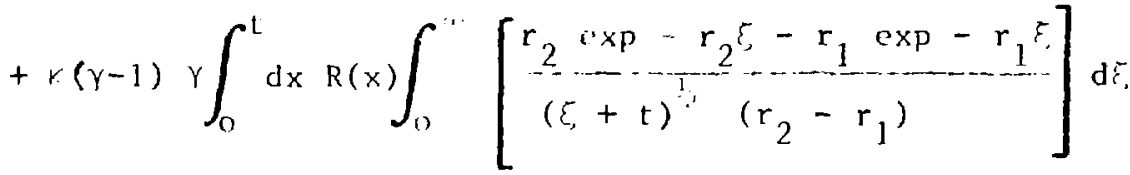

$$
\begin{aligned}
& +k(\gamma-1) \gamma \int_{0}^{t} d x R(x) \int_{0}^{(x)}\left[\begin{array}{c}
r_{2} \exp -r_{2} \xi_{1}-r_{1} \exp -r_{1} \xi_{1} \\
\left(\xi_{1}+t-x\right) \\
\left(r_{2}-r_{1}\right)
\end{array}\right] d \xi
\end{aligned}
$$

Finally, we write the fourth term as

$$
\begin{aligned}
I_{4}=k^{2}(\gamma-1)^{2} & \int_{0}^{\prime r} d r_{1} \int_{r_{1}}^{x_{1}}\left[\begin{array}{c}
r_{2} \exp -r_{2} \xi_{2}-r_{1} \exp -r_{1} \xi_{2} \\
\left(r_{2}-r_{1}\right)
\end{array}\right] \\
& {\left[\begin{array}{c}
r_{2} \exp -r_{2} r_{1}-r_{1} \exp -r_{1} \xi_{1} \\
r_{2}-r_{1}
\end{array}\right] M\left(\xi_{1}, \xi_{2}\right) }
\end{aligned}
$$

where

$$
\begin{gathered}
M\left(\xi_{1}, \xi_{2}\right)=\frac{1}{4} \int_{0}^{t} d t \int_{0}^{t} d s_{2} \int_{0}^{t} d s_{1} R\left(t-s_{2}-t_{1}+s_{1}\right) \\
\\
\left(r_{1}+s_{1}\right)^{3 / 2}\left(\xi_{2}+s_{2}\right)^{3 / 2}
\end{gathered}
$$

We note that $\mathrm{I}_{4}$ is symmetris under the interchange of $\varepsilon_{1}$ and $\xi_{2}$ and thus only the symmetrir part of $M\left(\varepsilon_{1}, \varepsilon_{2}\right)$ can contribute to $I_{4}$. We rewrite $\mathrm{M}$ as 


$$
\begin{aligned}
& M\left(\varepsilon_{1}, \varepsilon_{2}\right)=\frac{1}{4} \int_{0}^{t} d z \int_{0}^{t} d s_{2} \int_{0}^{t-z} d s_{1} \quad \begin{array}{ll}
R\left(z-s_{2}+s_{1}\right) \\
\left(\xi_{1}+s\right)^{3 / 2}\left(r_{2}+s_{2}\right)
\end{array} \\
& =\frac{1}{4} \int_{0}^{t} d z \int_{0}^{t} d s_{2} \int_{y}^{t} d x-\ldots r_{2}^{R\left(x-s_{2}\right)}
\end{aligned}
$$

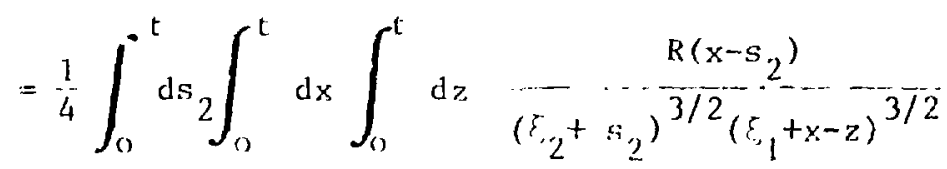

$$
\begin{aligned}
& =\frac{1}{2} \int_{0}^{-t} d \int_{0}^{t} d x \frac{R\left(x_{2}-s_{2}\right)}{\left(\xi_{2}+s_{2}\right)}\left[\begin{array}{c}
\frac{1}{3 / 2} \\
\left.-\frac{1}{1 / 2}-\frac{3}{\left(\xi_{1}+x\right)}\right]
\end{array}\right]
\end{aligned}
$$

Now transform variables, letting

$$
\begin{aligned}
& y_{1}=\frac{x-s_{2}}{\sqrt{2}} \quad x=\frac{y_{2}+y_{1}}{\sqrt{2}} \\
& y_{2}=\frac{x+s_{2}}{\sqrt{2}}-s_{2}=\frac{y_{2}-y_{1}}{\sqrt{2}} \\
& M\left(\varepsilon_{1}, \xi_{2}\right)=\frac{1}{2} \int_{0}^{L / \sqrt{2}} \mathrm{dy}_{1} \mathrm{R}\left(\sqrt{2} \mathrm{y}_{1}\right) \int_{1}^{\sqrt{2 \mathrm{t}-y_{1}}} \frac{\mathrm{dy} 2}{\left(\xi_{2}+\frac{\mathrm{y}_{2}-\mathrm{y}_{1}}{\sqrt{2}}\right)^{3 / 2}} \\
& {\left[\frac{1}{\xi_{1}}-\frac{1}{\left(\xi_{1}+\frac{y_{2}+y_{1}}{\sqrt{2}}\right)^{T}}\right]} \\
& +\frac{1}{2} \int_{-t / \sqrt{2}}^{0} d_{1} R\left(\sqrt{2 y_{1}}\right) \int_{-y_{1}}^{\sqrt{2} t+t+y} 1 \frac{d y_{2}}{\left(\xi_{2}+\frac{y_{2}-y_{1}}{\sqrt{2}}\right)^{3 / 2}}
\end{aligned}
$$




$$
\left.\left[\begin{array}{cc}
1 & - \\
1 & \left(1+\frac{1}{y_{2}+\bar{y}_{1}}\right. \\
1 & -\sqrt{2}
\end{array}\right)\right]
$$

Now we san do the y. integrals. In particular the inderinite intigrial

$$
{ }^{2} \int\left(\begin{array}{c}
\mathrm{dy} y_{2} \\
\left(\xi+\frac{y_{2}-y_{1}}{\sqrt{2}}\right) \\
-3 / 2
\end{array}\right.
$$

and

$$
\begin{aligned}
& \frac{1}{2} \int \frac{d y_{2}}{\left(\xi_{2}+\frac{y_{2}-y_{1}}{\sqrt{2}}\right)^{3 / 2}\left(\xi_{1}+\frac{y_{2}-y_{1}}{\sqrt{2}}\right)^{\frac{1}{3}}}
\end{aligned}
$$

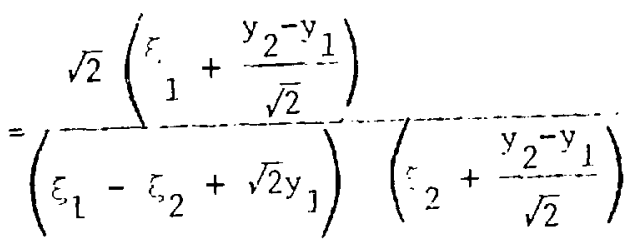

Then

$$
\begin{aligned}
& M\left(\varepsilon_{1}, \xi_{2}\right)=-\int_{0}^{t} \operatorname{dy} k(y) \frac{1}{\xi_{1}}\left[\begin{array}{cc}
1 \\
\left(\xi_{2}+t-y\right)^{\frac{1}{2}} & \varepsilon_{2}^{2}
\end{array}\right] \\
& -\int_{0}^{t} d y \operatorname{R}(y) \frac{1}{\varepsilon_{1}}\left[\frac{1}{\left(\xi_{2}+t\right)^{1}}-\frac{1}{\left(\xi_{2}+y\right)^{1}}\right]
\end{aligned}
$$




$$
\begin{aligned}
& +\int_{0}^{t} d y R(y) \frac{1}{\left(\xi_{2}-\frac{1}{\varepsilon_{2}}+y\right)}\left[\frac{\left(\xi_{1}+t\right)^{\frac{1}{3}}}{\left(\xi_{2}+t-y\right)^{\frac{1}{2}}}-\frac{\left(\xi_{1}+y\right)^{\frac{1}{2}}}{\xi_{2}^{\frac{1}{2}}}\right] . \\
& +\int_{0}^{t} d y R(y) \frac{1}{\left(\xi_{1}-\xi_{2}-y\right)}\left[\frac{\left(\xi_{1}+t-y\right)^{\frac{1}{2}}}{\left(\xi_{2}+t\right)^{\frac{1}{2}}}-\frac{\xi_{1}^{\frac{1}{2}}}{\left(\xi_{2}+y\right)^{\frac{1}{2}}}\right]
\end{aligned}
$$

Since we are concerned only with the symmetric part of $M$, we write

$$
\begin{aligned}
M_{1}\left(\xi_{1}, \xi_{2}\right) & =\frac{1}{\left(\xi_{1}-\xi_{2}+y\right)}\left[\frac{\left(\xi_{1}+t\right)^{\frac{1}{5}}}{\left(\xi_{2}+t-y\right)^{\frac{1}{2}}}-\frac{\left(\xi_{1}+y\right)^{\frac{3}{2}}}{\xi_{2}^{\frac{1}{2}}}\right] \\
& +\frac{1}{\left(\xi_{1}-\xi_{2}-y\right)}\left[\frac{\left(\xi_{1}+t-y\right)^{\frac{1}{2}}}{\left(\xi_{2}+t\right)^{\frac{3}{5}}}-\frac{\xi_{1}^{\frac{1}{2}}}{\left(\xi_{2}+y\right)^{\frac{1}{2}}}\right]
\end{aligned}
$$

and consider its symmetric part.

$$
\begin{aligned}
\operatorname{Sym}\left(M_{1}\right) & =\frac{1}{2}\left[M_{1}\left(\xi_{1}, \xi_{2}\right)+M_{1}\left(\xi_{2}, \xi_{1}\right)\right] \\
& =\frac{1}{2\left(\xi_{1}-\xi_{2}+y\right)}\left[\frac{\left(\xi_{1}+t\right)^{\frac{1}{2}}}{\left(\xi_{2}+t-y\right)^{\frac{1}{2}}}-\frac{\left(\xi_{2}+t-y\right)^{\frac{1}{2}}}{\left(\xi_{1}+t\right)^{\frac{1}{2}}}\right] \\
& +\frac{1}{2\left(\xi_{1}-\xi_{2}+y\right)}\left[\frac{\xi^{\frac{1}{2}}}{\left(\xi_{1}+y\right)^{\frac{1}{2}}}-\frac{\left(\xi_{1}+y\right)^{\frac{1}{2}}}{\xi_{2}^{\frac{3}{2}}}\right] \\
& +\frac{1}{2\left(\xi_{1}-\xi_{2}-y\right)}\left[\frac{\left(\xi_{1}+t-y\right)^{\frac{3}{2}}}{\left(\xi_{2}+t\right)^{\frac{1}{2}}}-\frac{\left(\xi_{2}+t\right)^{\frac{1}{3 /}}}{\left(\xi_{1}+t-y\right)^{\frac{1}{2}}}\right]
\end{aligned}
$$




$$
+\frac{1}{\left(\xi_{1}-\frac{1}{z_{c}-y}\right)}\left[\begin{array}{cc}
\left(\varepsilon_{2}+y\right)^{\prime} & \varepsilon^{\prime} \\
\left(\xi_{1}\right) & \left(\varepsilon_{2}+y\right)^{\prime}
\end{array}\right]
$$

$o r$

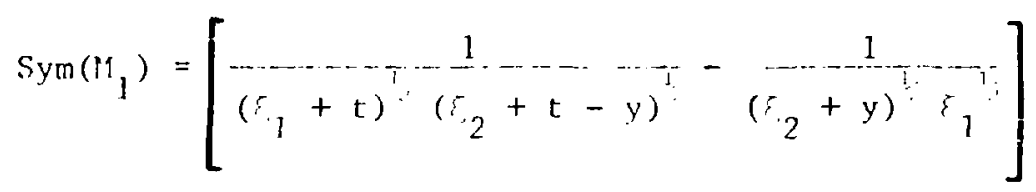

Then

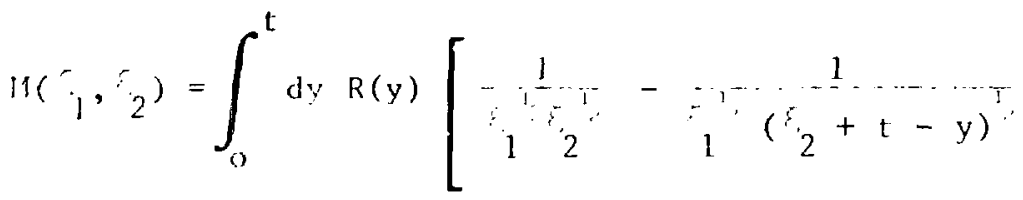

$$
\begin{aligned}
& {\left[\begin{array}{c}
\gamma_{1}\left(\xi_{2}+t\right) \\
\left.r_{1}+t\right)\left(\xi_{2}+t-y\right)
\end{array}\right]}
\end{aligned}
$$

Inserling this expression into $1_{4}$, we derive

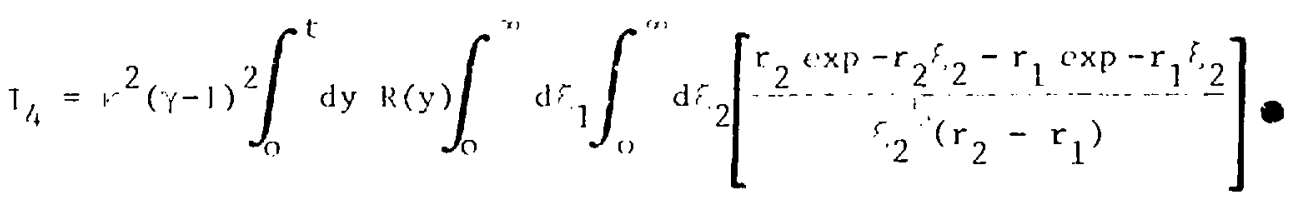

$$
\begin{aligned}
& {\left[\frac{r_{2} \exp -r_{2} \xi_{2}-r_{1} \exp -r_{1} \xi_{1} 1}{\xi_{1}^{\frac{1}{2}}\left(r_{2}-r_{1}\right)}\right]} \\
& +r^{2}(y-1)^{2} \int_{0}^{t} d y \quad n(y) \int_{0}^{x} d \cdot \int_{0}^{\prime \prime} d r_{2}\left[\begin{array}{c}
r_{2} \exp -r_{2} r_{2}-r_{1} \exp -r_{1} r_{2} \\
\left(r_{2}+t-y\right)\left(r_{2}-r_{1}\right)
\end{array}\right] \text { • } \\
& {\left[\begin{array}{c}
r_{2} \exp -r_{2} r_{2}-r_{1} \operatorname{xxp}-r_{1} r_{1} \\
\left(\xi_{1}+t\right) \\
\left(r_{2}-r_{1}\right)
\end{array}\right]}
\end{aligned}
$$




$$
\begin{aligned}
& -k^{2}(\gamma-1) \int_{0}^{t} d y R(y) \int_{0}^{\alpha} d \xi_{2}\left[\frac{r_{2} \exp -r_{2} \xi_{2}-r_{1} \exp -r_{1} \xi_{2}}{\left(\xi_{2}-t-y\right)\left(r_{2}-r_{1}\right)}\right] \\
& {\left[\frac{r_{2} \exp -r_{2} \xi_{1}-r_{1} \exp -r_{1} \xi_{1}}{\varepsilon_{1}\left(r_{2}-r_{1}\right)}\right]} \\
& -\kappa^{2}(\gamma-1) \int_{b}^{t} \mathrm{dy} R(y) \int_{0}^{\infty} \mathrm{d} \xi_{2}\left[\frac{r_{2} \exp -\mathrm{r}_{2} \xi_{2}-r_{1} \exp -r_{1} \xi_{2}}{\left(\xi_{2}+t\right)\left(r_{2}-r_{1}\right)}\right] \\
& {\left[\frac{r_{2} \exp -r_{2} \xi_{1}-r_{1} \exp -r_{1} \xi_{1}}{\xi_{1}^{1 / p}\left(r_{2}-r_{1}\right)}\right]}
\end{aligned}
$$

Performing the $\xi$ integrals wherever possible yields

$$
\begin{aligned}
& I_{4}=\pi k^{2}(\gamma-1)^{2}\left(\frac{r_{2}-r_{1}}{r_{2}-r_{1}}\right)^{2} \int_{0}^{t} d y R(y) \\
& -\sqrt{\pi \kappa^{2}}(\gamma-1)^{2}\left(\frac{r_{2}^{3}-r_{1}}{r_{2}-r_{1}}\right) \int_{0}^{t} d y R(y) \int_{0}^{r_{j}} d \xi\left[\frac{r_{2} \exp -r_{2} \xi-r_{1} \exp -r_{1} \xi}{(\xi+t-y)^{\frac{1}{z}}\left(r_{2}-r_{1}\right)}\right] \\
& -\sqrt{\pi} \kappa^{2}(\gamma-1)^{2}\left(\frac{r_{2}^{\frac{3}{2}}-r_{1}}{r_{2}-r_{1}}\right) \int_{0}^{t} d y R(y) \int_{0}^{m} d \varepsilon\left[\frac{r_{2} \exp -r_{2} \xi_{1}-r_{1} \exp -r_{1} \xi_{j}}{(\xi+t)^{\frac{1}{2}}\left(r_{2}-r_{1}\right)}\right] \\
& +\kappa^{2}(\gamma-1) \int_{0}^{t} d y R(y) \int_{0}^{a_{2}} d \xi_{1} \int_{0}^{\infty} d \xi_{2}\left[\frac{r_{2} \exp -r_{1} \xi_{2}-r_{1} \exp -r_{1} \xi_{2}}{\left(\xi_{2}+t-y\right)\left(r_{2}-r_{1}\right)}\right] \text {. }
\end{aligned}
$$




$$
\left[\begin{array}{c}
r_{2} \exp -r_{2} r_{1}-r_{1} \exp -r_{1} \xi_{1} \\
\left(r_{1}+t\right)\left(r_{2}-r_{1}\right)
\end{array}\right]
$$

Putting all the results for $\mathrm{I}_{2}, \mathrm{I}_{3}$, I/4 together and using

$$
\begin{aligned}
& {\left[\begin{array}{c}
r_{2}-r_{1} \\
-r_{2}-r_{1}
\end{array}\right]=\frac{1}{\text { r. } / i l} \text {, we writi }}
\end{aligned}
$$

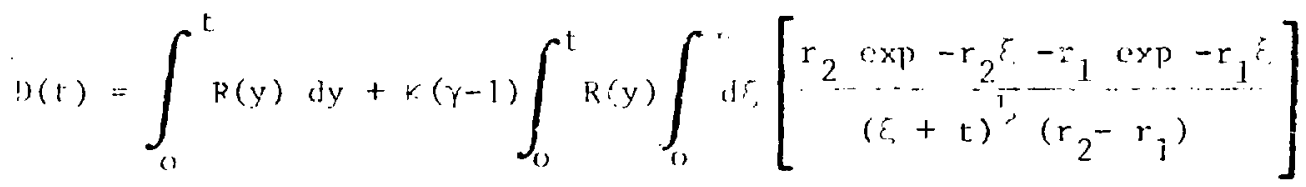

$$
\begin{aligned}
& +r(\gamma-1) \int_{0}^{t} R(y) d y \int_{0}^{r} d \xi\left[\frac{r_{2} \exp -r_{2} \xi_{,}-r_{1} \exp -r_{1} \xi^{\prime}}{(\xi+t-y)^{\frac{1}{2}}\left(r_{2}-r_{1}\right)}\right] \\
& +r^{2}(\gamma-1)^{2} \int_{0}^{t} R(y) d y \int_{0}^{\prime \prime} d r \cdot \int_{0}^{m} d \xi_{2} \\
& {\left[\begin{array}{c}
r_{2} \exp -r_{2} \xi_{2}-r_{1} \exp -r_{1} \varepsilon_{2} \\
\left(\xi_{2}+t-y\right) \\
\left(r_{2}-r_{1}\right)
\end{array}\right]\left[\begin{array}{c}
r_{2} \exp -r_{1} \xi_{1}-r_{1} \exp -r_{1} \varepsilon_{1} \\
\left(\xi_{1}+t\right)\left(r_{2}-r_{1}\right)
\end{array}\right]}
\end{aligned}
$$

Now Lhis expression is only analytic in $B$ over the interval $\frac{1}{9}<\beta=\frac{1}{2}$ and cannot be extended over any larger region. $1 \mathrm{t}$ can be put into an equivalent form which can he continued. To do this, we note that for 8 in the above interval,

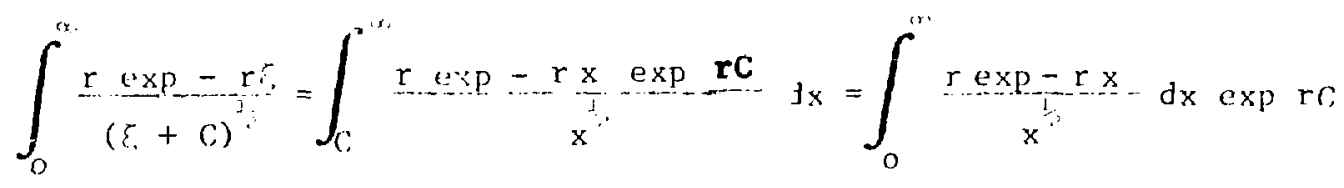




$$
\begin{aligned}
& \int_{0}^{r \exp -r x} d x \exp r C=\sqrt{\pi} r^{\frac{1}{2}} \exp r C \\
& -\exp r c \int_{0}^{c} \frac{r \cos -r x}{x}
\end{aligned}
$$

Thus, an equivalunt form for D(t) is

$$
\begin{aligned}
D(t) & =\int_{0}^{t} R(y) d y+r(\gamma-1) h(t) \int_{0}^{t} R(y) d y \\
& +r(\gamma-1) \int_{0}^{t} R(y) h(t-y) d y \\
& +r^{2}(\gamma-1)^{2} h(t) \int_{0}^{t} R(y) h(t-y) d y
\end{aligned}
$$

where

$$
\begin{aligned}
h(t)= & \sqrt{ }\left(r_{2} \exp r_{2}{ }^{\prime} r_{1} \exp r_{1} t\right) /\left(r_{2}-r_{1}\right) \\
& -\int_{0}^{1}\left[\begin{array}{ccc}
r_{2} & r_{2}(t-x)-r_{1} \exp r_{1}(t-x) \\
\cdots & \left(r_{2}-r_{1}\right)
\end{array}\right] d x .
\end{aligned}
$$

In this form, $D(t)$ is analytic for all physical values of $R$ and ain be continued to these values. Thus equasion 4.14 represents a general expression for the particle diffusivity subject to the particular set of initial conditions we have chosen.

\subsection{Evaluation of Particle Diffusivity for Several Special Cases}

We can carry our analysis further in several interesting special cases. We shall cont inue to employ Tchen's hypothesis concerning the equivalence of the various fluid correlation functions when possible. 
In Chapter III, we defined a correlation time $t_{c}$. We can also define a particle response time ${ }_{r}$ by

$$
\frac{1}{t_{r}}=\max \left[\left|\operatorname{Rea} 1\left(r_{1}\right)\right|,\left|\operatorname{Real}\left(r_{2}\right)\right|\right] \text {. }
$$

If we assume $t_{c} \ll t_{r}$, and also $t_{c} \ll t$, then the diffusion process becomes Markovian and we write

$$
R(y)=2 D_{M} \delta(y)
$$

where $D_{M}$ is a constant. We cannot assume $D_{M}$ equal $D_{F}$, the passive saclar diffusivity, because the correlation function $R(y)$ is not equivalent to the Lagrangian correlation in the case $t_{c} \ll t_{r}$ (see Chapter III). Inserting this form of $R(y)$ into equation 4.14, we find

$$
D(t)=D_{M}(1+\kappa(\gamma-1) h(t))^{?}
$$

This is the diffusion coefficient we would calculate for particles executing Brownian-like movement in a fluid with Stokes and Basset damping forces.

Another special case we consider is the diffusivity at early times, when $t \ll t_{r}$. In this limit,

$$
h(t) \rightarrow \frac{1}{k}-2 t^{\frac{1}{2}}
$$

and 


$$
\begin{aligned}
& D(t)=\gamma^{2} \int_{0}^{t} R(y) d y-k(\gamma-1) 2 t^{1} \int_{0}^{t} R(y) d y \\
& -k(\gamma-1) 2 \int_{0}^{t} R(y)(t-y)^{\frac{1}{3}} d y \\
& -k^{2}(\gamma-1)^{2} \frac{2}{k} t^{\frac{1}{2}} \int_{0}^{t} R(y) d y \\
& -k^{2}(\gamma-1)^{2} \frac{2}{k} \int_{0}^{t} R(y) d y(t-y)^{\frac{1}{2}} \\
& =\gamma^{2} \int_{0}^{t} R(y) d y-2 k \gamma(\gamma-1) t^{\frac{1}{2}} \int_{0}^{t} R(y) d y \\
& -2 k \gamma(\gamma-1) \int_{0}^{t} R(y)(t-y)^{\frac{1}{2}} d y \text {. }
\end{aligned}
$$

Thus, for early times, the particle diffusivity is approximately $\gamma^{2}$ times the fluid diffusivity. Since $\gamma=\frac{3 \rho_{F}}{2 \rho_{p}+\rho_{F}}$, we see that, for early times, heavy particles $\left(\rho_{p}>\rho_{F}\right)$ diffuse more slowly than the fluid and lighter particles diffuse more quickly than the fluid. At the other extreme, we consider the diffusivity for long time. In this limit

$$
h(t) \rightarrow 0
$$

and

$$
\lim _{t \rightarrow \infty} D(t)=\int_{0}^{\infty} R(y) d y=D_{F}
$$

That is, for long times the particle diffuses at the same rate as the 
fluid, in perfect agreement with the results of Chapter III. 


\section{CHAPTER $V$}

\subsection{Introduction of Shears into the Equation of Motion}

In this chaptor we want to further generalize the particle equation of motion 2.6. In Chapter $1 \mathrm{I}$ we obtained the body forces on the particle under the following assumption. Consider a sphere of fluid whose radius is many particle radji. Then, in the absence of the particle, we assumed that the fluid velocicy in this sphere could be approximated by some function $U_{i}(t)$ which varied in time, but was uniform over the entire sphere of fluid at any instant of time. We then introduced a particle into the center of such a sphere which perturbed the fluid velocity. The force laws of Chapter II were calculated by solving for this perturbed fluid velocity, finding the stress tensor at the surface of the particle, and integrating the normal component of the stress over the surface of the particle.

The program for this chapter is similar with the following modification. We shall now assume that the unperturbed fluid velocity in any sphere many particle radii in extent is given by

$$
u_{i}\left(x_{i}, t\right)=u_{i}(t)+\sigma_{i j}(t) x_{j}
$$

where $x_{j}$ is the position vector relative to the conter of the sphere. We employ the Einstein convention of summation over repeated indices throughout this chapter. $\sigma_{i j}(t)$ represents the shear tensor $\frac{\partial u_{i}}{\partial x_{j}}$ and is thus a traceless tensor; this follows from the divergence condition. Otherwise, $\sigma_{i j}(t)$ is an arbitrary stochastic function of time. The main assumption we shall make is that the shears are small in 
the sinst.

$$
R^{2}\left(g_{i j}(t)\right)^{2} \leftrightarrow\left(U_{i}(t)\right)^{2}
$$

for any choive of indices, Here $R$ is the particle ridius. 'This slight change of notation (in all previous chapters $R_{0}$ was used for the particle radius) shnuld lead co no confusion lor the fluid correlations R(t) will not appear in this chapter. The meaning of this assumption is that the fluid velocity does not change much over distances of the order of sevoral particle radii. Thus we expect that the fluid velocity around the particle and perturbed by the presence of the partiale will differ only slightly from a "zeroth order" perturbed fluid velocity calculated in the absence of shears.

In the next few paragraphs we will out ine a procedure for caloulating the force on the particle assuming the shours vanish. Then we will discuss the extension of this procedure to include the presence of shears. We sha1? assume that the fluid is characterized by high frequencies of oscillation. We begin our discussion by cxamining the consequences of this assumption.

Suppose that our fluid were totally inviscid (i.e. the viscosity $v=0)$. 'The fluid velocity would then be the solut $\downarrow$ on of the inviscid Navier-Stokes equations which ran be written 


$$
\frac{\partial(\operatorname{cur} 1}{\partial t} \frac{\vec{P}}{\partial t}=\operatorname{cur} 1[\overrightarrow{\mathrm{P}} \times \operatorname{cun} 1 \overrightarrow{\mathrm{P}}]
$$

$$
\operatorname{div} \overrightarrow{\mathrm{P}}=0
$$

with boundary conditions at the surface of the sphere

$$
\lim _{r \rightarrow R} \vec{r} \bullet \vec{P}=0
$$

( $\vec{n}$ belng the unt surface vector of the sphere)

and at infinity

$$
\lim _{r \rightarrow \infty} \vec{P}=u_{i}(t)
$$

(As we have stated, we now assume that the shears at infinity vanish.) Allowing for the presence of viscosity modifies this solution. However, as Landau and Lifshit $\mathrm{z}^{(19)}$ show, on page 91 , if the frequency of oscillation of the fluid w is high, the fluid velocity at distances from the sphere large compared with the quantity

$$
\delta=\sqrt{\downarrow / \omega}
$$

rapidly becomes $\overrightarrow{\mathrm{P}}$ the itrviscid solution. The region close to the sphere is called the boundary layet and $\delta$ is called the boundary layer thickness. We sha1l assume that the frequency is high enough that $\delta \ll R$.

Let us write the total fluid velocity as the sum of the inviscid solution $\vec{P}$ and a correction due to the presence of viscosity $Q$. The 
sum $(\vec{P}+\vec{Q})$ obeys the Navier-Stokes equat tons

$$
\begin{aligned}
& \left.\frac{\partial(\vec{P}+\dot{Q})}{\partial t}+l(\vec{P}+\vec{Q}) \circ \operatorname{grad}\right](\vec{P}+\vec{Q})=-\frac{1}{\rho_{F}} \operatorname{grad} \psi+v(\vee \circ \nabla)(\vec{P}+\vec{Q}) \\
& V \cdot(\vec{P}+\vec{Q})=0
\end{aligned}
$$

with houndary conditions at the surface of the sphere

$$
\lim _{r \rightarrow R}(\vec{p}+\vec{Q})=0
$$

and at infinity

$$
\lim _{r \rightarrow \infty}(\vec{P}+\vec{Q})=u_{1}(t)
$$

We write the pressure $\psi=\psi_{I}+\psi_{I I}$ where $\psi_{I}$ 1s, the pressure assoclated with the inviscid solution. By definition

$$
\frac{\partial \vec{P}}{\partial t}+(\vec{P} \cdot \operatorname{grad}) \vec{P}=-\frac{1}{\rho_{F}} \operatorname{grad} \psi_{I}
$$

(taking the curl of this equation leads to the alternate form of the inviscid equations 5.2 )

$$
\text { div } \vec{B}=0
$$

Thus $Q$ satisfies

$$
\begin{aligned}
\frac{\partial \vec{Q}}{\partial t} & +[\vec{P} \bullet \operatorname{grad}] \vec{Q}+[\vec{Q} \bullet \operatorname{grad}] \vec{P}+[\vec{Q} \bullet \operatorname{grad}] \vec{Q} \\
& =-\frac{1}{\rho_{F}} \operatorname{grad} \psi_{I I}+v(\nabla \bullet \nabla) \vec{Q}+v(\nabla \bullet \nabla) \vec{P}
\end{aligned}
$$


Now the boundary conditions on $\vec{P}$ at the surface of the sphere are that the normai component of $\overrightarrow{\mathrm{P}}$ vanishes. However, $\vec{P}+\vec{Q}$ must totally vanish at the surface and so, at the surface, the tangential component of $\vec{Q}$ must equal the negative tangential component of $\vec{P}$. At infinity, $\vec{Q}$ must vanish. However we know that at distances greater than $\delta$ from the surface of the sphere, $\vec{Q}$ is nearly zero. In fact, if we applied the boundary condition

$$
\lim _{r \rightarrow 10 \delta+R} \vec{Q}=0
$$

we would not alter the calculated velocity $\vec{Q}$ signiflcantly. On the basis of this discussion, we can estimate the various terms of equation

\section{3 as follows.}

Everywhere, $\overrightarrow{\mathrm{P}}$ varles appreciably in space over distance of the order of $R$. Within the boundary layer, $\vec{Q}$ varies appreclably in the normal direction over distances of order $\delta$ (changing from a value of order $|\overrightarrow{\mathrm{P}}|$ to zero), but varies appreciably in the tangential direction only over distances of order R. Taking the magnitude of $\overrightarrow{\mathbf{p}}$ at the surface as an estimate of the magnitude of the velocities in the system and denoting this quantity as $|\vec{P}(R)|$, we see that

$$
\begin{aligned}
& \frac{\partial \vec{Q}}{\partial t} \sim|\overrightarrow{\mathrm{P}}(\mathrm{R})| \omega \sim|\overrightarrow{\mathrm{P}}(\mathrm{R})| \frac{\nu}{\delta^{2}} \\
& (\nabla \circ \nabla) \overrightarrow{\mathrm{Q}} \sim|\overrightarrow{\mathrm{P}}(\mathrm{R})| \frac{1}{\delta^{2}} \\
& (\nabla \circ \nabla) \overrightarrow{\mathrm{P}} \sim|\overrightarrow{\mathrm{P}}(\mathrm{R})| \frac{1}{\mathrm{R}^{2}}
\end{aligned}
$$


The operator $(P+Q) \circ g r a d$ represents differentiation in the direction of the velocity. Within the boundary layer, the velocity is nearly tangential and so

$$
\begin{aligned}
& {[(\overrightarrow{\mathrm{P}}+\vec{Q}) \circ \operatorname{grad}] Q \sim|\overrightarrow{\mathrm{P}}(\mathrm{R})|^{2} \frac{1}{\mathrm{R}}} \\
& {[\overrightarrow{\mathrm{Q}} \circ \operatorname{grad}] P \sim|\overrightarrow{\mathrm{P}}(\mathrm{R})|^{2} \frac{1}{\mathrm{R}}}
\end{aligned}
$$

Now let us suppose that the Reynolds number

$$
\frac{|\vec{P}(R)| R}{v} \ll \frac{R^{2}}{\delta^{2}}
$$

We can allow the fluid to have a quite sizable Reynolds number (even of order $\frac{R}{\delta}$ ) and still satisfy this requirement. Thus, to lowest order in $\delta / R$, the equations 5.3 reduce to

$$
\begin{gathered}
\frac{\partial \vec{Q}}{\partial t}=-\frac{1}{\rho} \operatorname{grad} \psi_{I I}+(V \cdot V) \vec{Q} \\
V \cdot \vec{Q}=0
\end{gathered}
$$

within the boundary layer and the equations vanish outride the boundary 1ayer to this order. This latter statement is a justification of the comment that the boundary conditions at infinity might be just as well applied at a surface

$$
r=R+10 \delta
$$

(Note that there is no sperial signifirance to the choice $10 \mathrm{\delta}$. This could read $5 \delta$ or $50 \delta$ just as well.) 
From the linearity of equations 5.4 we may draw the following conclusion. $\vec{Q}$ is a linear function of the inviscid solution $\vec{p}$ evaluated at the surface of the particle. Furthermore, we note that the inviscid solution $\vec{P}$ is established with the speed of sound which, in a truty incompressible fluid, is infinite.

Suppose we write the boundary conditions on $\vec{P}+\vec{Q}$ in the form

$$
\lim _{r \rightarrow \infty}(\vec{P}+\vec{Q})=\sum_{n} \vec{c}^{(n)} H\left(t-t_{n}\right)
$$

where $H$ is the leaviside function and where $\vec{C}^{(n)}$ is a constant vertor. The velocity field $\vec{P}$ may be written as the superposition of steady states

$$
\vec{p}\left(x_{1}, t\right)=\sum_{n} \vec{p}^{(n)}\left(x_{i}\right) H\left(t-t_{n}\right)
$$

where $\vec{p}^{(n)}\left(x_{i}\right)$ is the steady-state inviscid solution which satisfies the boundary conditions at infinity

$$
\lim _{r \rightarrow \infty} \vec{p}(n)=\vec{C}^{(n)}
$$

The inviscid equations in steady state reduce (in the absence of shears) to

$$
\begin{aligned}
& \operatorname{div} \vec{p}^{(n)}=0 \\
& \operatorname{cur1} \vec{p}^{(n)}=0
\end{aligned}
$$

Thus, we may note that $\overrightarrow{\mathrm{P}}^{(n)}$ is a linear function of $\overrightarrow{\mathrm{C}}^{(n)}$. Now the equations for $\vec{Q}\left(x_{i}, t\right)$ are linear so that $\vec{Q}$ is a linear 
function of its boundary conditions, the function $\vec{p}$ evaluated at the surface of the particle. But these boundary conditions are linear functions of $\ddot{C}^{(n)}$ and so finally we can write $\dot{Q}$ as a superposition

$$
\vec{Q}\left(x_{i} t\right)=\sum_{n} Q^{(n)}\left(x_{i}, t\right)
$$

where $\vec{Q}^{(n)}$ is a solution of equations 5.4 satisfying the boundary and initial conditions

$$
\begin{aligned}
& \lim _{r \rightarrow R} \vec{Q}^{(n)}\left(x_{i}, t\right)=-\vec{P}^{(n)}(R) H\left(t-t_{n}\right) \\
& \lim _{r \rightarrow \infty} \vec{Q}^{(n)}\left(x_{i}, t\right)=0
\end{aligned}
$$

The prossure $\psi_{I I}^{(n)}$ is also linear in $c^{(n)}$. However, the pressure $\psi_{I}$ is not linear in $\vec{C}$. This is easily seen for the case we consider, for $\overrightarrow{\mathrm{P}}$ represents a potential flow and Bermulli's law applies. Writing $\phi\left(x_{i}, t\right)$ as the scalar potential, we have

$$
\frac{\partial \phi}{\partial t}+\frac{1}{2} \vec{p} \circ \vec{p}+\frac{\psi}{\rho_{F}}=0
$$

(See Landau and Lifshitz ${ }^{19}, \dot{p} \cdot 19$ ).

We can now construct the stress tensor

$$
\Sigma_{i j}=-\left(\psi_{I}+\psi_{I I}\right) \delta_{i j}+v \rho_{k}\left(\frac{\partial\left(P_{i}+Q_{i}\right)}{\partial x_{j}}+\frac{\partial\left(P_{j}+Q_{j}\right)}{\partial x_{i}}\right)
$$

From the previous discussion, we can write the stress tensor as the surn of two parts 


$$
\Sigma_{i j}=-\psi_{I} \delta_{1 j}+\sum_{n} \Sigma_{i j}^{(n)}
$$

where

$$
\sum_{i j}^{(n)}=-\left[\psi_{I I}^{(n)} \delta_{i j}-v p\left(\frac{\partial\left(P_{i}^{(n)}+Q_{i}^{(n)}\right)}{\partial x_{j}}+\frac{\partial\left(P_{j}^{(n)}+Q_{j}^{(n)}\right)}{\partial x_{i}}\right)\right]
$$

and where $\Sigma_{i j}^{(n)}$ is linear in the vector $c_{i}^{(n)}$ which represents the boundary conditions. The force on the particle is

$$
F_{i}=\int_{\delta}\left[\psi_{1} n_{j} \delta j\right] d \phi+\oint_{\delta} \sum_{n}\left(-\Sigma_{i j}^{(n)}{ }_{r_{j}}\right) d \phi
$$

where $\eta$ is the outward directed unit surface vector and 8 is the surface of the sphere.

Let us now pause to summarize our results. We have found that the force on a particle in a rapidly oscillating fluid may be written as the sum of two parts. The first part, the surface integral of the inviscid pressure, is exactly the total force which would be exerted on the particle if the fluid viscosity were zero. The second part is a linear function of the fluid velocity far from the particle. That part may be found by constructing the velocity fields $P_{i}^{(n)}\left(x_{i}\right)$ and $Q_{i}{ }^{(n)}\left(x_{i}, t\right)$ in the nanner described and superposing.

Now we would like to extend this procedure to include the presence of shears in the unperturbed fluid velocity. Proceeding as before, we run into two difficulties. The Inviscid steady-state solution with 
external boundary conditions given by equation 5.1 is not established with the speed of sound to the extent that the external shears represent vorticity because vorticity in an inviscid fluid can be altered only by the movement of fluid. Thus, the effect of vorticity far from the particle is not instantaneously felt at the particle.

We can circumvent this difficu. -y by supposing that the shears are convected past the particle at a rate which is large with respect to the rate of growth of the boundary layer. For example, the time required to convect the shears past the particle from a point ten particle radii distant is

$$
t_{c} \sim \frac{10 R}{u}
$$

The thickness of the boundary layer is estimated as

$$
\delta \sim \sqrt{v t}
$$

and so the time $t_{b}$ for $\delta$ to grow to be one particle radius is

$$
t_{b} \sim \frac{R^{2}}{v}
$$

Requiring $t_{c} \ll t_{b}$ means

$$
\frac{10 R}{u} \ll \frac{R^{2}}{v}
$$

or

$$
10<\frac{R \mathrm{u}}{\mathrm{V}} \quad \text { (The Reynolds number) }
$$


Clearly, we must have a large Reynolds number of relative flow to satisy our assumption. As mentioned previously, it is possible to assume a large Reynolds number and still use equation 5.4 .

The second difficulty is that the antisymmetric part of the shears is not cur 1-free. Thus the inviscid velocity distribution is not derivable from a scalar potential, but is rather the solution of a non-linear vector equation. We shall have to prove that the inviscid solution is. still a linear function of the boundary conditions 5.1 . The next section is concerned with this problem.

\subsection{The Inviscid Problem}

We will find it useful to work in spherical coordinates. In this system, the coordinates are $(r, \theta, \phi)$ which are related to the cartesian coordinates $(x, y, z)$ by

$$
\begin{aligned}
& x=r \sin \theta \cos \phi \\
& y=r \sin \theta \sin \phi \\
& z=r \cos \theta
\end{aligned}
$$

The unit basis vectors are $(\hat{r}, \hat{\theta}, \hat{\phi})$ which form a right-handed orthogonal set: They are related to the cartesian basis vectors ( $\hat{i}, \hat{j}, \hat{k}$ ) by

$$
\begin{aligned}
& \hat{\mathbf{r}}=i \sin \theta \cos \phi+\hat{j} \sin \theta \sin \phi+k \cos \theta \\
& \hat{\theta}=\hat{i} \cos \theta \cos \phi+\hat{j} \sin \theta \sin \phi-k \sin \theta \\
& \hat{\phi}=-\hat{i} \sin \phi+\hat{j} \cos \phi
\end{aligned}
$$

The components of a vertor $\vec{A}$ with respect to the spherical basis set are written

$$
\vec{A}=\hat{\mathbf{r}} \mathbf{A}_{\mathbf{r}}+\hat{\theta A_{\theta}}+\hat{\phi} A_{\phi}
$$


The problem we addross in this section can be stated as follows. We consider a spherical particle fixed at the origin of coordinates in a fluid. Far from the particle, the fluid velocity has a specified form. We seek the steady-state solution $\vec{Q}_{0}$ which is established everywhere on time scales in which viscous effects in the fluid are negligible. The Navier-Stokes equations for steady-state inviscid flow are

$$
\begin{gathered}
\operatorname{curl}\left[\vec{Q}_{0} \times \operatorname{curl} \vec{Q}_{0}\right]=0 \\
\operatorname{div} \vec{Q}_{0}=0
\end{gathered}
$$

One boundary condition is that the normal component of $\vec{Q}_{0}$ must vanish at the surface of the sphere. The escond boundary condition concerns the limiting form of $\vec{Q}_{0}$ far from the ephere and we shall want to discuss this in some detail.

In particular, we want to understand what we mean by "far from the sphere". Certainly we mean far enough away that the presence of the sphere can be neg1ected. In the absence of shears, an exact solution of these equations which satisfies the boundary conditions is

$$
\vec{P}=u\left[\hat{r} \cos \theta\left(1-R^{3} / r^{3}\right)-\hat{\theta} \sin \theta\left(1+R^{3} / 2 r^{3}\right)\right]
$$

In the absence of both the shears and the particle, the solution is simply

$$
\overrightarrow{\mathrm{U}}=\mathrm{u}(\hat{\mathbf{r}} \cos \theta-\hat{\theta} \sin \theta)
$$


Thus, by the time we are ten particle radii away from the sphere, the difference in these two fields is of order $10^{-3}$.

On the other hand, far from the sphere must also mean a disrance $\vec{x}$ such that

$$
\left|\sigma_{i j} x_{j}\right| \ll\left|u_{i}\right|
$$

That is, we want the perturbation due to the presence of shears to be sma11. Because we have assumed the shears to be small, these two concepts of "farness" are simultaneously realizable. Anytime we speak of infinity, we may for practical purposes consider a distance of ten particle radii to be a good approximation.

We have already pointed out that $\vec{P}$ is a zeroth order solution to our problem (order in which the shears vanish). The next order of solution must be first order in the quantity $\sigma_{i j^{*}}$ Writing

$$
\vec{Q}_{0} \cong \vec{P}+\vec{l}
$$

and recognizing that $\operatorname{cur} 1 \overrightarrow{\mathrm{P}}$ vanishes identicallv, the equations for $\vec{\Gamma}$ to lowest order are

$$
\begin{gathered}
\operatorname{cur1}(\overrightarrow{\mathrm{P}} \times \operatorname{cur} 1 \vec{\Gamma})=0 \\
\operatorname{div} \vec{\Gamma}=0
\end{gathered}
$$

with boundary conditions that the normal component of $\vec{\Gamma}$ vanishes at the surface of the sphere and that 
$\lim _{r \rightarrow \infty} \Gamma_{j} \rightarrow{ }^{i j} x_{j}$

Equations 5.5 are linear in $i^{5}$ and the boundary conditions are linear in ${ }_{i j}$. Thus we may consider the part of $l^{p}$ due to symmetric shears

$$
s_{i j}=\frac{1}{2}\left(\sigma_{i j}+\sigma_{j i}\right)
$$

and the part due to the antisymmetric shears

$$
a_{i j}=\frac{1}{2}\left(\sigma_{i j}-\sigma_{j i}\right)
$$

separately. The part of $\vec{r}$ due to $s_{i j} ; \vec{s}$, is not difficult to find. In fact, $s$ is curl-free far from the particle and so is curl-free everywhere. Thus $\vec{S}$ is derivable from a potential. It is easy to verify that $\overrightarrow{\mathrm{S}}$ is given, in index notation, by

$$
s_{i}=s_{i j} x_{j}\left(1+\frac{2 R^{3}}{r^{3}}\right)-s_{j k} x_{j} x_{k} x_{i}\left(\frac{j R^{3}}{r^{5}}\right)
$$

The part of $\vec{\Gamma}$ due to the antisymetric shears, which we w:ll $\operatorname{call} \vec{A}$, is not so easily found. We begin by writing the boundary conditions at infinity in the form

$$
\vec{u}=\hat{u k}+\vec{\Omega} \times \vec{r}
$$

where

$$
\Omega_{i} \equiv-\frac{1}{2} \varepsilon_{i j k}{ }^{a j k}
$$


and $\varepsilon_{i j k}$ is the Levi-Civita tonsor completeiy antisymmetric in three dimensions. The equations for $\ddot{A}$ are now

$$
\begin{gathered}
\operatorname{curl}(\vec{P} \times \operatorname{cor1} \vec{A})=0 \\
\operatorname{div} \vec{A}=0
\end{gathered}
$$

with boundary conditions

$$
\begin{aligned}
& \lim _{r \rightarrow R} A_{r}=0 \\
& \lim _{r-m \times} \vec{A}=\vec{\Omega} \times \vec{r}
\end{aligned}
$$

From these equations, we immediately notice that $\lambda$ is independent of the sign and magnitude of $u$. Thus $\lambda$ must be an even function of the vector $\hat{\mathbf{k}}$.

We also note that the vector $\vec{P} \times$ curl $\vec{A}$ has vanishing cur 1 and so can be written as the gradient of some scalar function $x$.

$$
\overrightarrow{\mathrm{P}} \times \operatorname{cur} 1 \overrightarrow{\mathrm{A}}=\operatorname{grad} x
$$

Furthermore, it follows that

$$
\overrightarrow{\mathrm{P}} \cdot \operatorname{grad} x=0
$$

This means that $X$ is constant along the streamlines of $\vec{p}$ and we can use this to calculate $x$. To be explicit, we can calculate $x$ in $a$ plane far upstream of the sphere (from the boundary condtions) and then use the stream1lnes of $\vec{p}$ to map $X$ over al1 space. 
Thus, in tha plani $z=-x$, we can write

$$
\begin{aligned}
& \text { grad } x=1 \mathrm{im} \quad \vec{p} x \operatorname{cour} \dot{\Lambda} \\
& \text { ax } \\
& =211 \mathrm{k} \times \hat{?}
\end{aligned}
$$

In this plane,

$$
x=211 r \sin \theta\left(\sin \sin \phi-s_{2} \cos \phi\right)
$$

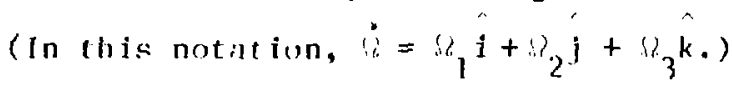

On the ather hand,

$$
0=p \cdot \operatorname{grad} x=\left(1-R^{3} / r^{3}\right) \cos \theta \quad \frac{\partial x}{\partial r}-\left(1+\frac{\left.R^{3} / 2 r^{3}\right)}{r} \sin \theta \frac{\partial x}{\partial \theta}\right.
$$

I'hus, $x=x(f, p)$ where

$$
P=\sqrt{r^{3}-R^{3}} \text { sin } 11
$$

This is not a surprising result, for the streamlines of $\vec{p}$ are the lines given by the intersections of the surfaces

$$
B=\text { constant }
$$

and

$$
\text { 小 }=\text { constant }
$$

In the plane $z=-x, \quad \therefore=r \sin \theta$, so that we can write $x$ in terms of the invariants $\beta$ and $t$ everywhere as

$$
x=2 U \sqrt{\frac{r^{3}-R^{3}}{r}} \sin \theta\left(s_{1} \sin \phi-s_{2} \cos \phi\right)
$$


Now we note that $x$ is independent of $\Omega_{3}$, the romponent of $\overrightarrow{\Omega_{3}}$ parallel to the incoming flow at infinity. The part of $\vec{\wedge}$ proportional to $\Omega_{3}$ is relatively easy to find. Assuming $i_{1}$ and $\Omega_{2}$ vanish, we have

$$
\vec{P} \times \operatorname{curl} \vec{A}=0
$$

The most general solution of this equation is

$$
\operatorname{curl} \vec{A}=f(r, \theta, \phi) \vec{P}
$$

Taking the divergence of hoth sides, and noting that div rurl of any vertor vanishes, we dertve

$$
\text { div } \mathrm{E}(r, \theta, \phi) \vec{p}=0
$$

Since div $\vec{p}=0$, we can write

$$
\vec{p} \cdot \operatorname{grad} f(r, \theta, \phi)=0
$$

Thus we can find $f(r, \theta, \phi)$ in exactly the same manner as we found $x$. In the plaue $z=-\infty$,

$$
\lim _{z \rightarrow-\infty} f(r, \theta, \phi)=2 \Omega_{3} / v
$$

and over a11 space, $f(r, \theta, \phi)$ must be the same. Thus

$$
\operatorname{cur} 1 \vec{A}=\frac{2 s i}{U} \quad \vec{P}
$$

Now we argue that $\vec{A}$ must be independent of the $\phi$ coordinate. 
This must be so, for cur1 $\vec{A}$ has this property and the boundary conditions on $\vec{A}$ have this property. In component form, then,

$$
\begin{aligned}
& \frac{\hat{r}}{r^{2} \sin \theta} \frac{\partial}{\partial \theta}\left(r \sin \theta A_{\phi}\right)=2 \hat{r} \Omega_{3}\left(1-R^{3} / r^{3}\right) \cos \theta \\
& \frac{\hat{\theta}}{r \sin \theta} \frac{\partial}{\partial r}\left(r \sin \theta A_{\phi}\right)=2 \hat{\theta} \Omega_{3}\left(1+R^{3} / 2 r^{3}\right) \sin \theta \\
& \frac{\hat{\phi}}{r}\left[\frac{\partial}{\partial r}\left(r A_{\theta}\right)-\frac{\partial}{\partial \theta}\left(A_{r}\right)\right]=0 \\
& \frac{1}{r^{2}} \frac{\partial\left(r^{2} \Lambda_{r}\right)}{r r}+\frac{1}{r \sin \theta} \frac{\partial\left(A_{0} \sin \theta\right)}{\partial \theta}=0
\end{aligned}
$$

The last equation follows from the divergence condition on $\vec{A}$. The solution which satisfies these equations and the boundary conditions is easily seen to be

$$
\begin{aligned}
& A_{r}=0 \\
& A_{\theta}=0 \\
& A_{\phi}=r\left(1-R^{3} / r^{3}\right) \sin \theta
\end{aligned}
$$

or

$$
\vec{A}=\left(1-R^{3} / r^{3}\right) \vec{\Omega} \times \vec{r}
$$

where $\vec{\Omega}=\Omega_{3} \hat{\mathrm{k}}$,

The part of $\vec{A}$ due to $\Omega_{1}$ and $\Omega_{2}$ satisfies 


$$
\begin{gathered}
\overrightarrow{\mathrm{P}} \times \operatorname{cur} 1 \vec{A}=\operatorname{grad} x \\
\operatorname{div} A=0
\end{gathered}
$$

where

$$
x=2 v \sqrt{\frac{r^{3}-R^{3}}{r}} \sin \theta\left(\Omega_{1} \sin \phi-\Omega_{2} \cos \phi\right)
$$

The boundary conditions are

$$
\lim _{r \rightarrow R} A_{r}=0
$$

and

$$
\lim _{r \rightarrow \infty} \vec{A}=\vec{\Omega} \times \vec{r}
$$

where

$$
\hat{s}=\Omega_{1} \hat{i}+\Omega_{2} \hat{i}
$$

We have not been able to solve these equations, although it is possible to isolate the $\phi$-dependence. In fact, substituting a solution of the form

$$
\begin{aligned}
\vec{\lambda} & =\hat{r} f(r, \theta)\left(\Omega_{2} \cos \phi+s_{1} \sin \phi\right) \\
& +\hat{\theta} g(r, 0)\left(\Omega_{2} \cos \phi+\Omega_{1} \sin \phi\right) \\
& +\hat{\phi} h(r, 0)\left(-\Omega_{2} \sin \phi+\Omega_{1} \cos \phi\right)
\end{aligned}
$$

will. cause the $\phi$ dependence to cirop out of the equations and the boundary conditions.

The lack of a solution for the last set of equations will not serlously hamper our calculations. We now have sufficient information about the inviscid solution to determine the force law to within one 
non-dimensional constint. The bases of this delermination are the known inviscid solutions, and certain argurients of symetry and tensor invariance. In the next seetion we will calculate the part of the foree on the particle which is linear in the far-field velocity given by equation 5.1. In scetion 5.4, we will calculate the part of the force dua to the inviscid pressure.

\subsection{The Viscous Forces}

We write the velocity firld at infinity as the superposition

$$
\sum_{n} \dot{c}(n)+\sigma_{i j}^{(n)} x_{j} \mid H\left(L-t_{n}\right)
$$

where the vector $i^{(n)}$ and the tensor $o_{i j}^{(n)}$ aro constants. Then the fluid velocity may bo writtill as tho sum of four terms

$$
\begin{aligned}
\sum_{n} p^{(n)} & +\vec{\Gamma}^{(n)} 1 H\left(1-t_{n}\right) \\
& +\sum_{n}\left[Q_{1}^{(n)}+i_{2}^{(n)}\right]
\end{aligned}
$$

Here $\left[p^{(n)}+\vec{p}^{(n)}\right]$ is the inviscid solution which satisfics the boundary ronditions at infinity

$$
\left.\lim _{r \rightarrow x)} \mid p^{(n)}+i^{(n)}\right\rceil=\stackrel{\rightarrow}{i}^{(n)}+\underset{i j}{(n)} x_{j}
$$

Furthermore, we have found, that $\vec{P}^{(n)}$ is linear in $\vec{C}^{(n)}$ and independent of $?_{i j}^{?(n)}$. Nlso $\vec{r}^{(n)}$ is linear in $\sigma_{i j}^{(n)}$. $\vec{\Gamma}^{(n)}$ may depend on the direction of $i^{(n)}$, but not on the magnitude of this vector. The sum $\left[\vec{Q}_{1}^{(n)}+\ddot{Q}_{2}^{(n)}\right]$ represents the correction to the inviscid 
solution due to tho presence of viscosity. These terms are the solution of equations 5.4 with boundary conditions at the sphere.

$$
\lim _{r \rightarrow R}\left[\vec{Q}_{1}^{(n)}+\vec{Q}_{2}^{(n)}\right]=-\lim _{r \rightarrow R}\left[\vec{P}^{(n)}+\vec{p}^{(n)}\right]
$$

Because equations 5.4 are 1 incar, we can define $\vec{Q}_{1}^{(n)}$ as the solution of equation 5.4 with boundary conditions at the sphere

$$
\lim _{r \rightarrow R} \vec{Q}_{1}^{(n)}=-\lim _{r \rightarrow R} \ddot{p}^{(n)}
$$

and simllarly $\vec{Q}_{2}^{(n)}$ as the solution of equations 5.4 with boundary conditions at the sphere

$$
\lim _{r \rightarrow R} \ddot{Q}_{2}^{(a)}=-\lim _{r \rightarrow R} \vec{\Gamma}(n)
$$

The pressure $\psi_{\text {It }}^{(n)}$ may be written as

$$
\psi_{I I}^{(n)}=\psi_{I I I}^{(n)}+\psi_{I !}^{(n)}
$$

where $\psi_{T I I}^{(n)}$ is the pressure field assoriated with the velocity $\vec{Q}_{1}^{(n)}$ and $\psi_{I V}^{(n)}$ is the pressure field associated with the velocity $\vec{Q}_{2}^{(n)}$. Now the quantities $\vec{p}^{(n)}, \vec{Q}_{1}^{(n)}$, and $\vec{\psi}_{1 I I}^{(n)}$ are all linear in $\overrightarrow{\mathrm{C}}^{(n)}$ and independent of $\sigma_{i j}^{(n)}$. The part of the stress tensor proportiona1 to these quantities leads to the forces on the sphere which are present even in the absence of shears - the Stokes and Basset forces. On the other band, $\vec{\Gamma}^{(n)}, \vec{Q}_{2}^{(n)}$ and $\psi_{\text {III }}^{(n)}$ are linear in $\sigma_{1 j}^{(n)}$ and independent of the magnitude of $\vec{C}^{(n)}$. The part 
of the stress tensor proportional to these quantities leads to the corrections to the force on the sphere due to the presence of shears. let us now attempt to construct these forces on the basis of tensor invariance.

Because $\stackrel{\Gamma}{\Gamma}^{(n)}, \vec{Q}_{2}^{(n)}$, and $\psi_{T V}^{(n)}$ are independent of the magnitude of $\vec{c}^{(n)}$, the available vectors for construction of the force must be constructed from the set

$$
\left(\delta_{i j}, \varepsilon_{i j k}, \sigma_{i j}^{(n)}, \sigma_{j i}^{(n)}, \hat{k}\right)
$$

or equivalently

$$
\left(\delta_{i j}, r_{i j k}, s_{i j}, s_{i}, \hat{k}\right)
$$

Here $\hat{k}$ denotes the direction of $\vec{c}(n)$.

The vectors we construct must be linear in $s_{i j}$ or $\Omega_{i}$. Forces constructed from $\hat{k}$ can depend on the vector only because $\vec{l}^{(n)}$ or $\hat{Q}_{2}^{(n)}$ does. Thus we must not allow a force depending upon the symmetric part of the shears $s_{i j}$ and $\hat{k}$ berause the part of $f^{(n)}$ (and hence $\vec{Q}_{2}^{(n)}$ ) due to $\mathrm{s}_{i j}$ is independent of $\hat{k}$. The part of $\vec{r}_{i}^{(n)}$ (and hence $\vec{Q}_{2}^{(n)}$ ) dependent on the antisymmetric shears, $s_{i}$, is invariant under the transformation $\hat{k}+\hat{-k}$ and thus so is the part of the force proportional to $\Omega_{i}$. The possible forces which satisfy all these restrictions are proportional to 

a) $\Omega_{i}$
b) $(\vec{\Omega} \circ \hat{k}) \hat{k}$
c) $(\vec{\Omega} \times \hat{k}) \times \hat{k}$

The last possibility, (c) can be neglected since it is a linear combination of the first vectors. It is now more convenient to write our two surviving vectors as
a) $\vec{\Omega}-(\vec{\Omega} \circ \hat{k}) \hat{k}$
b) $(\vec{\jmath} \circ \hat{k}) \hat{k}$

These st111 span the same set, but are not orthogonal. We now employ symmetry arguments to show that forces due to (a) and (b) must vanish.

First consider (a). This are forces due to $\Omega_{1}$ and $\Omega_{2}$, the components of $\vec{\Omega}$ along with $\hat{i}$ and $\hat{j}$ axes. Suppose $\vec{\Omega}=\Omega_{1} \hat{i}$ and consider the plane $x=0$. The part of $\vec{f}^{(n)}$ proportional to $\Omega_{1} \hat{1}$ has reflection symmetry across this plane, for it is calculated from equations 5.5 where $\vec{P}^{(n)}$ and the boundary conditions on $\vec{\Gamma}^{(n)}$ contain this symmetry. Also, the part of $\vec{Q}_{2}^{(n)}$ proportional to $\Omega_{1} \hat{i}$ contains this symmetry. There is no way to distinguish left from right along the $\hat{i}$ axis and so any force proportional to $\Omega_{1} \hat{i}$ must vanish. There is no real distinction in our problem between $\hat{i}$ and $\hat{j}$ and similar arguments apply to any force proportional to $\Omega_{2} \hat{j}$.

The mathematical basis of these arguments lies in the known $\phi$-dependence of $\vec{F}^{(n)}$. From equations 5.8 , with $\Omega_{2}=0$, we verify 
1.h. symmetry of refloction across the $x=0$ plane. Also. it is aisy lo verify from equations 5.4 that the part of $\dot{Q}_{2} n$ ) proportional to $\Omega_{1}$ has the liorm

$$
\hat{\theta g}(r, \theta, t) s_{1} \sin \phi+\ddot{\phi i h}^{\prime}(r, \theta, t) s_{1} \cos \phi .
$$

If wo use this form to actually construct the force, we find the force is proportional to the $\phi$ integral

$$
\int_{0}^{2 \pi} \sin \phi \cos \phi d \phi=0
$$

The sreond possibility for the force, proportional to $83^{k}$ is argued in a similar manner. The velocity fields $\vec{\Gamma}^{(n)}$ and $\ddot{Q}_{2}^{(n)}$ proportional to $\Omega_{3} \hat{k}$ will be symmetric under reflection in the $z=0$ plane. There is no way 10 distinguish left from right along the $\hat{k}-a x i s$ and so any force proportional to iz $\hat{k}$ must vanish.

The time-varying force due to $\vec{l}, \vec{Q}, \dot{\psi} \mathrm{f}$ is found by superposing the results fir $\vec{P}^{(n)}, Q^{(n)}, \psi_{I V}^{(n)}$. The superposing of zero forces wIll lead to no force. Hence we may conclude that there are no corrections to the forces on a particle, due to the presence of shears in the external velocity field, which are derivable from $\vec{P}^{\prime}, \vec{Q}, \psi_{1 V^{*}}$ This result is correct under the assumptions of a thin boundary laycr, small shears and a high Reynolds member of relative flow.

5.4 The Inviscid Pressure Forees

In this section we will calculate the forces on the particle due to the inviscid pressure $\psi_{I}$. These forces are exactly the total force which would he cxerted on the particle if the viscosity of the fluid 
vanished.

Suppose, to begin, that the houndary conditions at infinity are independent of time. The inviscid solution satisfies the steady-state equation

$$
\left[\left(\overrightarrow{\mathrm{P}}+\overrightarrow{I^{5}}\right) \circ \operatorname{grad}\right](\overrightarrow{\mathrm{P}}+\vec{\Gamma})=-\frac{1}{\rho_{F}} \operatorname{grad} \psi_{I}
$$

Using the vector identity valid for any vector $\vec{v}$

$$
\frac{1}{2} \operatorname{grad}(\vec{V} \circ \vec{V})=\vec{V} \times \operatorname{cur} 1 \vec{v}+(\vec{V} \circ \operatorname{grad}) \vec{V}
$$

we derive

$$
\frac{1}{2} \operatorname{grad}[(\overrightarrow{\mathrm{P}}+\overrightarrow{\vec{l}}) \cdot(\overrightarrow{\mathrm{P}}+\vec{\Gamma})]-(\overrightarrow{\mathrm{P}}+\vec{\Gamma}) \times \operatorname{cur}\left(\overrightarrow{\mathrm{P}}+\overrightarrow{\vec{\Gamma}^{\prime}}\right)=-\operatorname{grad}\left[\frac{\psi_{\mathrm{I}}}{\rho_{\mathrm{F}}}\right]
$$

We have seen that, if the stears are smal1, the sccond term may be approximated as $\vec{P} \times$ curl $\vec{\Gamma}$ and furthermore that

$$
\overrightarrow{\mathrm{P}} \times \operatorname{cur} 1 \ddot{\Gamma}=\operatorname{grad} x
$$

where $x=20 \sqrt{\frac{r^{3}-R^{3}}{r}} \sin \theta\left(\Omega_{1} \sin \phi-\Omega_{2} \cos \phi\right)$

Here $U$ is the constant translation velocity at infinity and $\Omega_{1}$ and $\Omega_{2}$ are derived from the constant shea" at infinity

$$
\begin{aligned}
& \Omega_{1}=-\frac{1}{2} \varepsilon_{1 j k} \sigma_{j k} \\
& \Omega_{2}=-\frac{1}{2} \varepsilon_{2 j k} \sigma_{j k}
\end{aligned}
$$


The equation can now bo written

$$
\operatorname{grad}\left[\frac{1}{2}(\vec{p}+\vec{r}) \circ(\dot{p}+\vec{p})-x+\frac{\psi}{\square}\right]=0
$$

and a first integration can be easily effected.

$$
\frac{\psi_{I I}}{\rho}-x+\frac{1}{2}(\vec{P}+\vec{r}) \circ(\vec{P}+\vec{r})=\text { constant }
$$

The steady-state force due to the inviscid pressure may be written

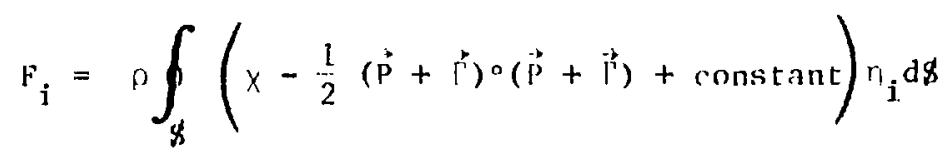

where $\$$ is the surface of the particle and ${ }^{\prime \prime}$, is the outward-directed unit normal vertor to this surface.

Now $x$ vanishes on $\phi$. Also, $\hat{r} \circ \vec{p}$ vanishes on $\$$ and $\hat{\phi} \circ \vec{p}$ vanishes everywhere. The surface integral of any symmetric function $[\theta$ vanishes. Since $\vec{p} \circ \vec{p}$ and a constant are both even functions of 0 , we find

$$
F_{i}=\rho \int_{\phi}\left(-P_{\theta} r_{\theta}\right) n_{j} d \&
$$

To derive this expression, we have also, neglected $\vec{i} \circ \vec{\Gamma}$ as being higher order in the shears. Writing

$$
\Gamma_{\theta}=s_{\theta}+\Lambda_{\theta}
$$

where $\vec{S}$ is the part of the inviscid solution proportional to the symmetric shears and $\vec{A}$ is the part of the inviscid solution proportional to the antisymmetric shears, we can define two corstants $\xi^{\prime}$ and $\lambda^{\prime}$ 


$$
\begin{aligned}
\xi^{\prime} s_{i j} u_{j} R^{3} & =\int_{\$}\left(P_{\theta} s_{\theta}\right) r_{j} d S \\
\lambda^{\prime} a_{i j}{ }^{\prime \prime}{ }^{3}{ }^{3} & =\int_{\$}\left(P_{\theta} A_{\theta}\right) n_{j} d \not S
\end{aligned}
$$

These are the two non-djmensional constants indetermined by the invariance arguments.

We can find $\xi^{\prime}$ hy assuming only $s_{33}$ and $U_{3}$ are non-vanishing. Then, on the surface of the sphere

$$
\mathrm{P}_{\theta} \mathrm{s}_{\theta}=\frac{9 R}{2} \quad \mathrm{~s}_{33} \mathrm{U}_{3}\left(\cos \theta-\cos ^{3} \theta\right)
$$

The portion of tha surface integral in the $\hat{k}(3)$ direction is

$$
\begin{aligned}
\int_{8} d \phi\left(P_{\theta} s_{\theta}\right)(\hat{n} \circ k) \\
=\int_{0}^{2 \pi} \sin \theta d \theta\left(\cos ^{2} \theta-\cos ^{4} \theta\right) \int_{0}^{2 \pi} d \phi\left(\frac{9 R^{3}}{2} s_{33} U_{3}\right) \\
=\frac{12 \pi}{5} R^{3} u_{3} s_{33}
\end{aligned}
$$

Thus $\xi^{\prime}=\frac{12 \pi}{5}$. The constant $\lambda^{\prime}$ could be determined in the same way except $A_{\theta}$ is not known. It will be left as an undetermined constant. The force on the particle due to the fluid, in steady-state is then

$$
F_{i}=-R^{3}\left[\xi^{\prime} s_{i j}+\lambda ' a_{i j}\right] u_{j}
$$


Now let us view the particle in a framework in which the particle moves through the fluid with a constant velocity $\overrightarrow{-} \vec{u}$. The fluid velocity at infinity is assumed to be " ${ }_{i j} x_{j}$. As we have seer, the fluid eserts a force

$$
\vec{F}=-R^{3}\left[s^{\prime}, s_{i j}+\lambda a_{i j} \mid u_{j}\right.
$$

on the particle. If the particle is to move with constant velocity, the net force on it must be zero. Hence we must exert a force $-\vec{F}$ on the particlo (hy means of a string, or perhaps by means of some electromagnetio process) to maintain its motion.

by Newton's siscond law, the particle exerts the negative of $\vec{F}$ on the fluid. In order to maintain the boundary conditiols at infinity, then, we must impose an external force on the fluid of $\mathrm{F}$. Then the momentum of the fluid remains ronstant, for there is no net force on the fluid. Sn far, we have been describing a completely steady-state situation.

Now suppose that $\vec{u}$ and ${ }^{i j}$ are allowed to vary in time. Because the time varying inviscid solution may be viewed as a sucression of steady states, we will still require the forces described in the steady-state configuration. There may now be additional forces. For if the total momentum of the fluid varies with $\vec{u}$ and $\sigma_{i j}$, then each successive steady state may have a different total fluid momentum. This change of momentum must be the result of either some force exerted by the particle on the fluid or of the action of some external force on 
the fluid.

Let us now calculate the total momentum $\pi_{i}$ of the fluid in the steady-state configuration where the particle velocity $\vec{u}$ and the far field fluid veloctey $\sigma_{i j} x_{j}$ are constant in time. Having $\Pi_{1}$, we know that the change in momentum from one steady state to the next must be the result of $a$ force equal to $\frac{d \Pi i}{d t}$. We can calculate $\Pi_{1}$ by carefully adding up the individual momenta of each tiny element of fluid. The veloctly distribution in the fluid is

$$
\overrightarrow{\mathbf{u}}-\overrightarrow{\mathbf{p}}+\overrightarrow{\mathrm{r}}
$$

The momentum is a linear function of the fluld veloclty and so the total. momentum is seen to been the sum of two terms. One term is the momentum contribution obtatned by adding the individual momenta of $\vec{u}-\vec{p}$, and this term will be a linear function of $\vec{u}$. This term is calculated in Landau and Lifshitz ${ }^{19}$ on pages 34 and 36 and is

$$
\frac{2}{3} \pi \rho_{F} R^{3} u_{1}
$$

The term which represents the contribution of $\vec{i}$ is linear in $\sigma_{i j}$. Like $\vec{\Gamma}$, it inay depend on the direction of $\vec{u}$, but not on its magnitude. This tomentum must also be Invartant under the transformation $\hat{k} \rightarrow-\hat{k}$ for $\vec{I}$ is invariant under this transformation. (Here $\hat{k}$ again represents the direction of $\vec{u}_{.}$) We see that the only avallable vectors from which to construct this momentum are

$$
\vec{\Omega}-(\vec{\Omega} \circ \hat{\mathrm{k}}) \hat{\mathrm{k}} \quad \text { and } \vec{\Omega}
$$


App1;ing the same symmetry arguments as we used in the previous section, we see that any momentum constructed froln these vectors must vanish. Thus

$$
\pi_{i}=\frac{2}{3} \pi \rho_{F} R^{3} u_{i}
$$

Now this is the same total momentun of the fluid that would be present in the absence of shears and the force on the fluid due to changes in $\pi_{i}$ is

$$
\frac{d \Pi_{i}}{d t}=\frac{2}{3} \pi \rho_{F} R^{3} \frac{d u_{i}}{d t}
$$

with is the virtual nass force. There are no additional virtual masslike forces due to the presence of shears.

If we now allow the particle to move with velocity $w_{i}$, the fluid velority at infinity to be ${ }^{11_{i}}+\sigma_{i j} x_{j}$, then the force $\sigma^{-}$the particle due to the presence of shears is

$$
F_{i}=-\rho R^{3}\left[\xi^{\prime} s_{1, j}+\lambda^{\prime} a_{i j}\right] v_{j}
$$

where $v_{j}=w_{j}^{-u_{j}}$ is the relative velocity. In terms of ${ }_{i j}$, we can write the force per unit mass due to the presence of shears as

$$
\frac{F_{i}}{\frac{4 \pi}{3} \rho_{p} R^{3}}=-\xi_{i j}(t) v_{j}(t)-\lambda \sigma_{j i}(t) v_{j}(t)
$$


$-118-$

$$
\text { where } \begin{aligned}
\xi_{0} & =\frac{3}{4 \pi}\left(\xi^{\prime}+\lambda^{\prime}\right)=\frac{9}{5}+\frac{3 \lambda^{\prime}}{4 \pi} \\
\lambda & =\frac{3}{4 \pi}\left(\xi^{\prime}-\lambda^{\prime}\right)=\frac{9}{5}-\frac{3 \lambda^{\prime}}{4 \pi}
\end{aligned}
$$


CHAPTER VI

6.1 Solution of the Equations of Motion with Shears

In this chapter we will investigate the influence of small

shears in the fluid velocity on the particle diffusivity. It will be seen that the Basset force plays no role in altering the results of this chapter and so we will neglect it in the particle equation of motion. The basic equation of motion for this chapter is

$$
\begin{aligned}
\left(\frac{d w_{1}}{d t}\right)_{p} & =\gamma\left(\frac{d u_{i}}{d t}\right)_{F}-\alpha\left(w_{1}-u_{1}\right) \\
& -\xi \sigma_{i j}\left(w_{j}-u_{j}\right)-\lambda \sigma_{j i}\left(w_{j}-u_{j}\right)
\end{aligned}
$$

We have once again indicated how the time derivatives are to be taken since it is now a matter of importance. In fact, we have

$$
\begin{aligned}
\left(\frac{d u_{i}}{d t}\right)_{F} & =\frac{\partial u_{i}}{\partial t}+u_{j} \frac{\partial u_{i}}{\partial x_{j}} \\
& =\frac{\partial u_{i}}{\partial t}+w_{j} \frac{\partial u_{i}}{\partial x_{j}}-\left(w_{j}-u_{j}\right) \frac{\partial u_{i}}{\partial x_{j}} \\
& =\left(\frac{d u_{i}}{d t}\right)_{p}-\sigma_{i j}\left(w_{j}-u_{j}\right)
\end{aligned}
$$

Our equation should then read 


$$
\begin{aligned}
\frac{d w_{1}}{d t} & =y \frac{d u_{1}}{d t}-\alpha\left(w_{1}-u_{1}\right)-\left(\gamma+\xi_{j}\right) \sigma_{1 j}\left(w_{j}-u_{j}\right) \\
& -\gamma \sigma_{j 1}\left(w_{j}-u_{j}\right)
\end{aligned}
$$

with the understanding that we measure all functions of time at position of the particle.

We will solve this equation as a perturbation problem. To zeroth order we neglect the shears. If we also set all initial conditions to zero, the lowest solution is

$$
w_{i}^{(0)}(t)=\gamma u_{1}(t)-\alpha(\gamma-1) \int_{0}^{t} u_{1}\left(t^{\prime}\right) \exp \alpha\left(t^{\prime}-t\right) d t^{\prime}
$$

as was derfved in Chapter III, equations 3.2. To the next order, our equation is

$$
\begin{aligned}
\frac{d w_{1}^{(1)}}{d t} & =\alpha w_{1}^{(1)}(t)-(\gamma+\xi)\left(w_{j}^{(0)}(t)-u_{j}(t)\right) \sigma_{i j}(t) \\
& -\gamma\left(w_{j}^{(0)}(t)-u_{j}(t)\right) \sigma_{j 1}(t)
\end{aligned}
$$

A solution of this equation is

$$
w_{1}^{(1)}(t)=-\int_{0}^{t} \exp \alpha\left(t^{\prime}-t\right) v_{j}\left(t^{\prime}\right)\left[(\gamma+\xi) \sigma_{i j}\left(t^{\prime}\right)+\lambda \sigma_{j 1}\left(t^{\prime}\right)\right]
$$

where

$$
v\left(t^{\prime}\right)=(\gamma-1)\left[u_{1}\left(t^{\prime}\right)-\alpha \int_{0}^{t^{\prime}} u_{1}\left(t^{\prime \prime}\right) \exp \alpha\left(t^{\prime \prime}-t^{\prime}\right) d t^{\prime \prime}\right]
$$


To lowest order then, the particle velocity can be written

$$
w_{1}(t)=w_{1}^{(0)}(t)+w_{1}^{(1)}(t)
$$

which is linear in the shears.

It is now possible to construct the correlation functions. Again, to lowest order in the shears, we can write in the 1direction

$$
\begin{aligned}
\overline{w_{1}\left(t_{1}\right) w_{1}\left(t_{2}\right)} & =\overline{w_{1}^{(0)}\left(t_{1}\right) w_{1}^{(0)}\left(t_{2}\right)}+\overline{w_{1}(0)\left(t_{1}\right) w_{1}{ }^{(1)}\left(t_{2}\right)} \\
& +\overline{w_{1}^{(1)}\left(t_{1}\right) w_{1}{ }^{(0)}\left(t_{2}\right)}
\end{aligned}
$$

Note that the next order, quadratic in the shears, is not simply $\overline{w^{(1)}\left(t_{1}\right) w^{(1)}\left(t_{2}\right)}$. In addition, there are terms representing the higher order of solution $w_{1}{ }^{(2)}(t)$ (terms 11 ke $w_{1}{ }^{(0)}\left(t_{1}\right) w_{1}{ }^{(2)}\left(t_{2}\right)$ $+\overline{w_{1}{ }^{(2)}\left(t_{1}\right) w_{1}{ }^{(0)}\left(t_{2}\right)}$ ) Also, there are additional terms in the force law itself which can be quadratic in the shears. The first term of equation 6.4 has been treated in Chapter III (see equation 3.9). Looking at our expresstons for $w_{1}{ }^{(1)}(t)$ and $w_{1}^{(1)}(t)$, we see that the last two terms involve correlations like $\overline{u_{1}\left(t_{1}\right) \sigma_{1 j}\left(t_{2}\right) u_{j}\left(t_{3}\right)}$ where $t_{3}$ may or may not equal $t_{2}$.

The force laws we derived in Chapter $V$ depend on the thinness of the boundary layer and the smallness of the shears. In turn, the thinness of the boundary layer is a consequence of the high frequency of the turbulent part of the fluid velocity. Thus, if the shears are 
sufficlently small, the force laws will be valld for both the mean and fluctuating parts of the shears. We are primarily concerned with the mean flow shears, for these w111 enable us to calculate particle diffustvity in problems 1ike turbulent pipe f1ow. Although the turbulence is neither homogeneous nor 1sotropic in this problem, it w111 be approximate1y so in the neighborhood of the particle if the particle is sma11 enough.

The fluctuating part of the shears leads to corrections to particle diffusivity due to the finite size of the particle. We sha11 have a few comments to make about these conditions in the last section of this chapter.

6.2 Particle Diffusivity in the Presence of Mean Flow Shears We can write the long-time particle diffusivity in the 1direction as

$$
\begin{aligned}
& D_{p}=\lim _{t \rightarrow \infty} \int_{0}^{t} d t^{\prime}\left[\overline{w_{1}^{(0)}(t) w_{1}^{(0)}\left(t^{\prime}\right)}+\overline{w^{(0)}(t) w_{1}^{(1)}\left(t^{\prime}\right)}\right. \\
& \left.+w^{(0)}\left(t^{\prime}\right) w^{(1)}(t)\right]
\end{aligned}
$$

to first order in the shears. Since the turbulence is assumed isotropic, we can write

$$
\overline{w_{1}(t) w_{1}\left(t^{\prime}\right)}=\frac{1}{3} \overline{w_{1}(t) w_{1}\left(t^{\prime}\right)}
$$

for any choices of superscripts. The first term of $D_{p}$ was calculated in 
Chapter III and was found to equal $D_{F}$, the passive scalar fluld diffusivity. Thus we can write, in tensur notation and using the summation convention,

$$
D_{p}=D_{F}+\frac{1}{3} \lim _{t \rightarrow \infty} \int_{0}^{t} d t^{\prime}\left(\frac{w_{1}^{(1)}(t) w_{1}^{(0)}\left(t^{\prime}\right)}{w_{1}^{(1)}\left(t^{\prime}\right) w_{1}^{(0)}(t)}\right)
$$

We also note that, in an isitrupts flutd

$$
R_{1 j}\left(t-t^{\prime}\right)=\overline{u_{1}(t) u_{j}\left(t^{\prime}\right)}
$$

is proportional to the Kronecker delta $\delta_{1 j}$. That is, given any $u_{i}(t)$, the probability of $u_{j}\left(t^{\prime}\right)$ must be same as the probability of $-u_{j}\left(t^{\prime}\right)$ if $j \neq 1$.

Now consider the correlation under the integral in equation 6.5. As noted previously, we see that all terms have the form

$$
\overline{u_{i}\left(t_{1}\right) \sigma_{1 j}\left(t_{2}\right) u_{j}\left(t_{3}\right)}=\sigma_{i j}\left(t_{2}\right) \overline{u_{i}\left(t_{1}\right) u_{j}\left(t_{3}\right)}
$$

since $\sigma_{i j}$ is assumed to be a mean flow shear. But the correlation Is then proportional to the Kronecker delta and we write

$$
\begin{aligned}
\sigma_{i j}\left(t_{2}\right) \overline{u_{1}\left(t_{1}\right) u_{j}\left(t_{3}\right)} & =R\left(t_{1}-t_{3}\right) \delta_{i j} \sigma_{i j}\left(t_{2}\right) \\
& =R\left(t_{1}-t_{3}\right) \frac{\partial u_{i}\left(t_{2}^{\prime}\right)}{\partial x_{i}} \\
& =0
\end{aligned}
$$

The last line follows from the incompressibility. Thus, to first order 
In the shears, the particle diffusivity in the presence of shears and based on the equation of motion 6.1 equals the passive scalar diffusivity.

Suppose now that we had Included the Basset Term in equation 6.1. If $\rho_{p} 1.75 \rho_{F}$, then $w_{i}(0)$ in equation 6.2 would be modified to read

$$
\begin{aligned}
w_{1}(0) & =\gamma u_{1}(t)-x(\gamma-1) \int_{0}^{t} d n u_{1}(n)_{0} \\
& \int_{0}^{\infty}\left[\frac{r_{2} \exp r_{2}(t-s)-r_{1} \exp r_{1}(t-s)}{2(s-n)^{3 / 2}\left(r_{2}-r_{1}\right)}\right] d s
\end{aligned}
$$

(see equation 4.9). It is now clear that the particle diffusivity will contain the same correlations $\overline{\sigma_{1 j}\left(t_{2}\right) u_{1}\left(t_{1}\right) u_{j}\left(t_{3}\right)}$,

and that they will vanish for the same reasons. By the process of analytic continuation described in Chapter IV, we see that the results of passive scalar diffusion will extend to arbitrary values of $\rho_{p} \cdot$

The results of this section can be summarized as follows. We consider a particle suspended in a turbulent fluld characterized by high frequencies of oscillation. We calculate the diffusivity of such a particle in the presence of small mean-flow shears, allowing for the presence of Stokes force, the virtual mass force, the Basset force and forces proportional to the shears. To first order in the shear strengths, we find the time-dependent particle diffusivity is unaffected by the presence of the mean-flow shears. 6.3 Comments on Fluctuating Shears

Tchen's neighborhood hypothesis assumes the spatial 
homogeneity of the fluid velocity in a region about the particle. We can relax this assumption slightly, allowing for the presence of smal1 Inhomgeneities, by assuming the presence of sma11, fluctuating shears in the fluid velocity. We still assume that the fluid velocity is statistically homogeneous, however. The presence of sma11, fluctuating shears will effect our diffusivity calculations in two ways. First, the additional forces due to the shears will alter the particle velocity correlation function, and hence the diffusivity. Returning to equations $6.2,6.3$, and 6.4 , and assuming that the shears are fluctuating quantities, we see that the particle velocity correlation will contain terms proportional to

$$
T\left(t_{1}, t_{2}, t_{3}\right)=\overline{u_{i}\left(t_{1}\right) \sigma_{i j}\left(t_{2}\right) u_{j}\left(t_{3}\right)}
$$

which is a triple correlation. Very little is known about correlations of this type.

The second way the fluctuating shears affect the diffusivity concerns the interpretation of particle diffusivity in terms of passive scalar diffusivicy. We have already noted that there exist two flufd velocity correlations of interest to us. One is the correlation along the motion of the fluid. This is the Lagrangtan correlation which we will denote

$$
R_{L}\left(t_{2}-t_{1}\right)=\frac{1}{3} \overline{u_{1}\left(t_{1}\right) u_{1}\left(t_{2}\right)} L
$$


in the shears, the particle diffusivity in the presence of shears and based on the equation of motion 6.1 equals the passive scalar diffusivity.

Suppose now that we had included the Basset Term in equation 6.1 . If $\rho_{p} 1.75 \rho_{F}$, then $w_{i}^{(0)}$ in equation 6.2 would be modified to read

$$
\begin{aligned}
w_{i}^{(0)}(t) & =\gamma u_{i}(t)-\kappa(\gamma-1) \int_{0}^{t} d n u_{i}(n) 0 \\
& \int_{0}^{\infty}\left[\frac{r_{2} \exp r_{2}(t-s)-r_{1} \exp r_{1}(t-s)}{2(s-n)^{3 / 2}\left(r_{2}-r_{1}\right)}\right] d s
\end{aligned}
$$

(see equation 4.9 ). It is now clear that the particle diffusivity w111 contain the same correlations $\overline{\sigma_{1 j}\left(t_{2}\right) u_{i}\left(t_{1}\right) u_{j}\left(t_{3}\right)}$,

and that they will vanish for the same reasons. By the process of analytic continuation described in Chapter IV, we see that the results of passive scalar diffusion will extend to arbitrary values of $\rho_{\mathrm{p}}$

The results of this section can be summarized as follows. We consider a particle suspended in a turbulent fluid characterized by high frequencies of oscillation. We calculate the diffusivity of such a particle in the presence of small mean-flow shears, allowing for the presence of stokes force, the virtual mass force, the Basset force and forces proportional to the shears. To first order in the shear strengths, we find the time-dependent particle diffusivity is unaffected by the presence of the mean-flow shears.

\subsection{Comments on Fluctuating Shears}

Tchen's neighborhood hypothesis assumes the spatial 
homoreneity of the fluid velocity in a region about the particle. We can relax this assumption slightly, allowing for the presence of small inhomgeneities, by assuming the presence of small, fluctuating shears in the fluid velocity. We still assume that the fluid velocity is statistically iomogeneous, however. The presence of small, fluctuating shears will effect our diffusivity calculations in two ways. First, the additional forces due to the shears will alter the particle velocity correlation function, and hence the diffusivity. Returning to equations $6.2,6.3$, and 6.4 , and assuming that the shears are fluctuating quantities, we see that the particle velocity correlation will contain terms proportional to

$$
T\left(t_{1}, t_{2}, t_{3}\right)=\overline{u_{i}\left(t_{1}\right) \sigma_{i j}\left(t_{2}\right) u_{j}\left(t_{3}\right)}
$$

which is a triple correlation. Very little is known about correlations of this type.

The second way the fluctuating shears affect the diffusivity concerns the interpretation of particle diffusivity in terms of passive scalar diffusivity. We have already noted that there exist two fluid velocity correlations of interest to us. One is the correlation along the motion of the fluid. This is the Lagrangian correlation which we will denote

$$
R_{L}\left(t_{2}-t_{1}\right)=\frac{1}{3} \overline{u_{i}\left(t_{1}\right) u_{i}\left(t_{2}\right)} L
$$


The other is the correlation of velocitles along the motion of the particle which we have denoted

$$
R\left(t_{2}-t_{1}\right)=\frac{1}{3} \overline{u_{i}\left(t_{1}\right) u_{i}\left(t_{2}\right)}
$$

Tchen ${ }^{(4)}$ assumed in his work that these two correlation are the same. However, based on the existence of small fluctuating shears, we can derive a more accurate relation between $R\left(t_{2}-t_{1}\right)$ and $R_{L}\left(t_{2}-t_{1}\right)$. This new relationship will enable us to reinterpret the particle diffusivity in terms of passive scalar diffusivity. The difference betreen Tchen's results and our results will also be proportional to the triple correlation $T\left(t_{1}, t_{2}, t_{3}\right)$.

Let us suppose that at time $t_{1}$ the particle is located at $x_{i}\left(t_{1}\right)$. At a later time $t_{2}$, we shall suppose the particle is located at $x_{1}\left(t_{2}\right)$. Also, at this time, a point which moves with the fluid and is coincident with the particle at time $t_{1}$ is now at $x_{i}^{\prime}\left(t_{2}\right)$. If the fluid velocities at $x_{i}\left(t_{2}\right)$ and $x_{i}{ }^{\prime}\left(t_{2}\right)$ are close enough, they are related by the shear $\sigma_{i j}\left(t_{2}\right)$

$$
u_{i}\left(x_{i}\left(t_{2}\right), t_{2}\right)=u_{i}\left(x_{i}\left(t_{2}\right), t_{2}\right)+\sigma_{i j}\left(t_{2}\right)\left[x_{i}\left(t_{2}\right)-x_{i}^{\prime}\left(t_{2}\right)\right]
$$

Thus we can write

$$
\begin{aligned}
u_{i}\left(x_{i}\left(t_{1}\right), t_{1}\right) u_{i}\left(x_{2}\left(t_{2}\right), t_{2}\right) & =u_{i}\left(x_{i}\left(t_{1}\right), t_{1}\right) u_{i}\left(x_{i}\left(t_{1}\right), t_{2}\right) \\
& +u_{i}\left(x_{i}\left(t_{i}\right), t_{1}\right) \sigma_{i j}\left(t_{2}\right) l_{j}
\end{aligned}
$$

where $\ell_{f}=x_{j}\left(t_{2}\right)-x_{j}{ }^{\prime}\left(t_{2}\right)$. Equation 6.6 cannot be true for all $t_{2}$, 
for eventually the vector $\ell_{j}$ must grow larger than distances over which the shear can be assumed constant. The idea is that $\ell_{j}$ will be small in time intervals for which the fluid velocities have large correlation.

Now we can write

$$
\ell_{j}=\int_{t_{1}}^{t} v_{j}(t) d t
$$

where $v_{j}$ is the relative velocity. To lowest order, we may use equation 6.2 and write

$$
\begin{aligned}
v_{j} & =(\gamma-1)\left[u_{j}(t)-\alpha \int_{0}^{t} u_{j}\left(t^{\prime}\right) \exp \alpha\left(t^{\prime}-t\right) d t^{\prime}\right] \\
& =(\gamma-1) \frac{d}{d t}\left[\int_{j}^{t} u_{j}\left(t^{\prime}\right) \exp \alpha\left(t^{\prime}-t\right) d t^{\prime}\right]
\end{aligned}
$$

Then

$$
\ell_{j}=(\gamma-1)\left[\int_{0}^{t} 2 d t^{\prime} u_{j}\left(t^{\prime}\right) \exp \alpha\left(t^{\prime}-t\right)-\int_{0}^{t} 1 t^{\prime} u_{j}\left(t^{\prime}\right) \exp \alpha\left(t^{\prime}-t\right)\right]
$$

Ensemble averaging equation 6.6 now yields the relation between the two correlation functions

$$
\begin{aligned}
3 R\left(t_{2}-t_{1}\right) & =3 R_{L}\left(t_{2}-t_{1}\right)+(\gamma-1) \int_{0}^{t_{2}} d t^{\prime} \exp \alpha\left(t^{\prime}-t_{2}\right) \cdot \\
& \overline{u_{i}\left(t_{1}\right) \sigma_{i j}\left(t_{2}\right) u_{j}\left(t^{\prime}\right)} \\
& -(\gamma-1) \int_{0}^{t} d t^{\prime} \exp \alpha\left(t^{\prime}-t_{1}\right) u_{i}\left(t_{1}\right){ }_{i j}\left(t_{1}\right) u_{j}\left(t^{\prime}\right)
\end{aligned}
$$

The last two terms involve the triple correlation $T\left(t_{1}, t_{2}, t^{\prime}\right)$. We 
have indicated that these correlations are to be taken along the motion of the particle, but this is not essential, for the difference between

$$
\overline{u_{1} \sigma_{1 j} u_{j}} \text { and } \overline{u_{1} \sigma_{1 j} u_{j}} L \text {. }
$$

is presumably one order higher in $\sigma_{1 f^{*}}$.

We close this section by proving that $R\left(t_{2}-t_{1}\right)$ is a symmetric function of $t_{2}-t_{1}$, demonstrating a few of the known properties of the triple correlation. Strictly speaking, the shears should be written

$$
\sigma_{1 j}\left(t_{2}\right)=\frac{\partial u_{1}\left(x_{2}\left(t_{2}\right), t_{2}\right)}{\partial x_{1}\left(t_{2}\right)}
$$

The operator $\frac{\partial}{\partial x_{1}\left(t_{2}\right)}$ means take the spatial derivative at the point $x_{1}\left(t_{2}\right)$ holding the time variable fixed. The operator $\frac{\partial}{\partial x_{1}\left(t_{1}\right)}$ is related to $\frac{\partial}{\partial x_{1}\left(t_{2}\right)}$ by

$$
\frac{\partial}{\partial x_{1}\left(t_{2}\right)}=\frac{\partial x_{1}\left(t_{1}\right)}{\partial x_{1}\left(t_{2}\right)} \frac{\partial}{\partial x_{1}\left(t_{1}\right)}
$$

But: we know

$$
x_{1}\left(t_{1}\right)=x_{1}\left(t_{2}\right)+\int_{t_{2}}^{t_{1}} w_{1}(t) d t
$$

so that $\frac{\partial x_{1}\left(t_{1}\right)}{\partial x_{1}\left(t_{2}\right)}=1$.

We shall now write $\frac{\partial}{\partial x}$ for the particle derivative with respect to the 
spatial variable at anv time.

Now consider $\overline{u_{i}\left(t_{1}\right) \sigma_{i j}\left(t_{2}\right) u_{j}\left(t^{\top}\right)}$. We write

$$
\begin{aligned}
u_{i}\left(t_{1}\right) \sigma_{i f}\left(t_{2}\right) u_{j}\left(t^{\prime}\right) & =u_{i}\left(t_{1}\right) \frac{\partial u_{1}\left(t_{2}\right)}{\partial x_{j}} u_{j}\left(t^{\prime}\right) \\
& =\frac{\partial}{\partial x_{j}} \frac{\left.u_{i}\left(t_{1}\right) u_{j}\left(t_{2}\right) u_{j}\left(t^{\prime}\right)\right)}{\left(u_{i}\left(t_{1}\right) u_{j}\left(t^{\prime}\right)\right)}
\end{aligned}
$$

The first term vantshes for reasons of homogenelty. The second term can be written

$$
\overline{u_{i}\left(t_{2}\right) \frac{\partial}{\partial x_{j}}\left(u_{i}\left(t_{1}\right) u_{j}\left(t^{\prime}\right)\right)}=u_{i}\left(t_{1}\right)_{j}\left(t^{\prime}\right) \frac{\partial u_{i}\left(t_{1}\right)}{\partial x_{j}}
$$

because of the incompressibility. Thus our result is

$$
\overline{u_{i}\left(t_{1}\right) \sigma_{i j}\left(t_{2}\right) u_{j}\left(t^{\prime}\right)}=-\overline{u_{i}\left(t_{2}\right) u_{j}\left(t^{\prime}\right) \sigma_{1 j}\left(t_{1}\right)}
$$

Thus we can write equation 6.7 in the form

$$
\begin{aligned}
R\left(t_{2}-t_{1}\right) & =R_{L}\left(t_{2}-t_{1}\right) \\
& +(\gamma-1) \int_{0}^{t} d t^{\prime} \exp \alpha\left(t^{\prime}-t_{2}\right) \overline{u_{1}\left(t_{1}\right) \alpha_{1 j}\left(t_{2}\right) u_{j}\left(t^{\prime}\right)} \\
& +(\gamma-1) \int_{0}^{t} \frac{1}{d t^{\prime}} \exp \alpha\left(t^{\prime}-t_{1}\right) \overline{u_{i}\left(t_{2}\right) \alpha_{i j}\left(t_{1}\right) u_{j}\left(t^{\prime}\right)}
\end{aligned}
$$


Since $R_{2}\left(t_{2}-t\right)$ is presumed to be symmetric fin $t_{2}^{-t_{1}}$ and the sum of the last two Integrals is also symmetric in $t_{2}-t$, we see that $R\left(t_{2}-t_{1}\right)$ is symmetrie in $t_{2}^{-t}{ }^{\text {* }}$ 


\subsection{T.Inearized Form Drag}

Up to this point, we have neglersed form drag in the partiale equation of motton. In general it is very difficult to include this non-linear force in diffusivity calculations, but there is a rase which can be trated easily. This is the case in which the particle moves through a turbulent medium with a large mean velocity.

Consider, for example, the problem of raindrops falling through the turbulent atmosphere under the Influence of grovity. Neglecting, forces due to the fluctuating fluid velocity, the raindrops will reach a terminal velocity which can be calculated by balancing the accoleration due to gravity and the deceleration due to stokes drag and form drag. There is no Basset force on a particle moving with constant velocity. The balance equation in the vertical direction reads

$$
6 \pi \rho_{F} V R_{O} V+\frac{1}{2} \pi \rho_{F} R_{O}{ }^{2} C_{D} V|V|=\frac{4 \pi R_{O}^{3}}{3}\left(\rho_{P}-\rho_{F}\right) g
$$

where $C_{D}=$ form drag constant (order one)

$$
\begin{aligned}
& g=\text { acceleration of gravity, }-980 \mathrm{~cm} \mathrm{sec}^{-2} \\
& R_{0}=\text { particle radius } \\
& v=\text { fluid kinematic visocity }
\end{aligned}
$$

The terminal velocity can be calculated now. Assuming the raindrop has a radius of $.1 \mathrm{~cm}$, the terminal veloctty is found to be

$$
v=-511 \mathrm{~cm} \mathrm{sec}^{-1}
$$


which is about 11 miles per hour.

The particle equation of motion is written

$$
\begin{aligned}
& \frac{d w_{i}}{d t}=\gamma \cdot \frac{d w_{1}}{d t}-\gamma\left(w_{1}-u_{1}\right)-\beta\left(w_{1}-u_{1}\right)\left|w_{i}-u_{1}\right| \\
& -k \int_{-\infty}^{t}\left[\frac{d w_{i}-\frac{d u_{1}}{d t^{b}}}{\sqrt{t-t^{2}}}\right] d t^{\prime}+g_{i}
\end{aligned}
$$

where

$$
\begin{aligned}
& B=\frac{3 \rho_{F} L_{D}}{4 R_{o}\left(2 \rho_{p}+\rho_{F}\right)} \\
& G_{1}=\frac{2\left(\rho_{P}-\rho_{F}\right)}{2 \rho_{p}+\rho_{F}} g_{1}
\end{aligned}
$$

and $\alpha, \gamma, \kappa$ have the same meaning as in Chapter IV. Let us choose our coordinate system so that the 3-axis points in the upward vertical direction $\left(g_{1}=\delta_{13} g\right)$. We define the vector $v_{1}$ as a vector whose magnitude is $|v|$ and which points in the negative $3-$ direction. We also define

$$
q_{i} \equiv w_{1}-v_{i}-u_{1}
$$

When the raindrop reaches terminal velocity, $q_{i}$ is the fluctuating relative particle velocity.

We now make the assumption that

$$
T=\frac{\left|q_{1}\right|}{|v|} \ll 1 \quad i=1,2,3
$$


In this case, we can linearize the form drag.

$$
\begin{aligned}
\left|w_{1}-u_{1}\right| & =\left|v_{i}+q_{i}\right|=\sqrt{q_{1}^{2}+q_{2}^{2}+\left(v+q_{3}^{2}\right.} \\
& \cong-v-q_{3}
\end{aligned}
$$

to first order in $\tau$. Also, to first order in $\tau$, the component equations 7.2 read

$$
\begin{aligned}
\frac{d q_{1}}{d t} & =(\gamma-1) \frac{d u_{1}}{d t}-(\alpha-\beta v) q_{1}-k \int_{-\infty}^{t} \frac{\frac{d q}{d t^{\prime}}}{\sqrt{t-t^{\prime}}} d t^{\prime} \\
\frac{d q_{2}}{d t} & =(\gamma-1) \frac{d u_{2}}{d t}-(\alpha-\beta v) q_{2}-k \int_{-\infty}^{t} \frac{\frac{d q_{2}}{d t^{T}}}{\sqrt{t-t^{\prime}}} d t^{\prime} \\
\frac{d q_{3}}{d t} & =(\gamma-1) \frac{d q_{3}}{d t}-(\alpha-2 \beta v) q_{3}-k \int_{-\infty}^{\frac{t}{\frac{d q_{3}}{d t^{\prime}}} d t^{\prime}} \\
& -[\alpha v+\beta v|v|-G]
\end{aligned}
$$

The last term in brackets in the equation for $q_{3}$ vanishes (equation 7.1). Thus, we see the equations of motion are forma11y the same equations we considered in Chapter IV. The diffusion coefficient is then given by equation 4.14 


$$
\begin{aligned}
D(t) & =\int_{0}^{t} R(y) d y+k(\gamma-1) h(t) \int_{0}^{t} R(y) d y \\
& +k(\gamma-1) \int_{0}^{t} R(y) h(t-y) d y \\
& +k^{2}(\gamma-1)^{2} h(t) \int_{0}^{t} R(y) h(t-y) d y
\end{aligned}
$$

where

$$
\begin{aligned}
h(t) & =\frac{\sqrt{\pi}}{r_{2}{ }^{-r_{1}}}\left[r_{2}^{\frac{1}{2}} \exp r_{2} t-r_{1}^{\frac{1}{2}} \exp r_{1}^{\frac{1}{2}}\right] \\
& -\int_{0}^{t}\left[\frac{r_{2} \exp r_{2}(t-x)-r_{1} \exp r_{1}(t-x)}{x^{\frac{1}{2}}\left(r_{2}-r_{1}\right)}\right] d x
\end{aligned}
$$

Now, however, $r_{1}$ and $r_{2}$ must be redefined. In the horizontal plane

$$
\begin{aligned}
& r_{1}=\frac{\kappa^{2} \pi-2 \alpha+2 \beta V}{2}-\frac{\sqrt{k^{2} \pi\left(\kappa^{2} \pi-4 \alpha+4 \beta V\right)}}{2} \\
& r_{2}=\frac{\kappa^{2} \pi-2 \alpha i \cdot 2 \beta V}{2}+\frac{\sqrt{k^{2} \pi\left(\kappa^{2} \pi-4 \alpha+4 \beta V\right)}}{2}
\end{aligned}
$$

In the vertical direction

$$
\begin{aligned}
& \mathbf{r}_{1}=\frac{\kappa^{2} \pi-2 \alpha+4 \beta V}{2}-\frac{\sqrt{k^{2} \pi\left(k^{2} \pi-4 \alpha+8 \beta V\right)}}{2} \\
& r_{2}=\frac{k^{2} \pi-2 \alpha+4 \beta V}{2}+\frac{\sqrt{3\left(k^{2} \pi-4 \alpha+8 \beta V\right)}}{2}
\end{aligned}
$$

Also, in equation 7.4 , we must be careful as we interpret the correlation function $R(y)$. This is stili the correlation of fluid 
velocity along the motion of the particle, but now is nothing like the Lagranglan correlation of fluid veloctties. Instead of assuming that the perticle approximately follows the fluid, we propose the following picture. We Imagine our particle moving rapidly through the fluid, passing from eddy to eddy in a time much shorter than the time it takes the turbulence $t$ /s change appreciably in any eddy. To a lowest order of approximation, we assume that the turbulence in an eddy does not change at all in the time it takes the particle to traverse the eddy. In this case, the correlation $R(t)$ can be expressed in terms of the two-point one-time spatial correlation functions. There are two of these - $f(r)$, the longitudina 1 correlation or correlation of velocities parallel to the vector joining the two points; and $g(r)$, the lateral correlation or correlation of velocities perpendicular to the vector joining the two points. The separation vector in this case is in the 3-direction and is given by $\vec{r}=\vec{v} t$. Keeping in mind that the turbulence is isotropic (so that $\overline{u_{3}{ }^{2}}=\overline{u_{1}{ }^{2}}=\overline{u_{2}{ }^{2}}=\frac{1}{3} \overline{u^{2}}$ ) we write for the particle velocity correlation function in the vertical direction

$$
R^{(11)}(t)=\frac{1}{3} \overline{u^{2}} \quad f(|v t|)
$$

and in the horizontal plane

$$
R^{(1)}(t)=\frac{1}{3} u^{2} g(|v t|)
$$


We can Improve on this model by allowing for slight changes in the turbulence during the particle's traverse. Following Peskin ${ }^{16}$, we assume the correlation functions can he written as the spatial correlation reduced by the Eulerian time correlation $E(t)$.

$$
R^{(I I)}(t)=\frac{1}{3} \bar{u}^{2} f(|v t|) E(t)
$$

and

$$
R^{(1)}(t)=\frac{1}{3} \bar{u}^{2} g(|v t|) E(t)
$$

Now there is a relationship between $g(x)$ and $f(r)$ in isotropic turbulence,

$$
g(r)=f(r)+\frac{r}{2} \frac{\partial f}{\partial r}
$$

(see Hinze 8, p.146). Furthermore, the work of Hay and Pasquili $1^{25}$ suggests that the Eulerian correlation function can be expressed in terms of the Lagrangian correlation function $R_{L}(t)$,

$$
E(t)=R_{L}(\lambda t)
$$

where $\lambda$ is a constant depending on $\overline{u^{2}}$. For atmosphere problems, $\lambda$ is found to be of order ten. (See Lumley and Panofsky ${ }^{26}$, pp. 199201). The correlation functions can now be written

$$
\begin{aligned}
& R^{(I)}(t)=\frac{1}{3} \overline{u^{2}} f(|v t|) R_{L}(\lambda t) \\
& R^{(1)}(t)=\frac{1}{3} \overline{u^{2}}\left(f(|v t|)+\frac{|v t:|}{2} \frac{\partial f}{\partial r} R_{L}(\lambda t)\right)
\end{aligned}
$$

Finally, let us assume some specific forms for the functions $f(r)$ and $R_{t}(t)$. For $f(r)$ we assume 


$$
f(r)=\exp -r / \ell_{c}
$$

where $\ell_{c}$ is the spatial correlation length. One might think of $\ell_{c}$ intuitively as the average size of an eddy. This form is consistent with measured data (Hinze ${ }^{4}$, p.187). Also, we shall assume

$$
R_{L}(t)=\exp -|t| / t_{L}
$$

where $t_{L}$ is the Lagrangian integral scale. This is the form used by most authors. (see Hinze ${ }^{4}$, p. $466, \operatorname{Csanady}^{27}$, p. $54, \operatorname{Levich}^{7}$ ). With these assumptions.

$$
\begin{aligned}
& R^{(I I)}(t)=\frac{1}{3} \overline{u^{2}} \exp -\frac{\lambda|t|}{t_{L}} \exp -\frac{|V t|}{l_{c}} \\
& R^{(1)}(t)=\frac{1}{3} \overline{u^{2}} \exp -\frac{\lambda|t|}{t_{L}}\left(1-\frac{|V t|}{2 l_{c}}\right) \exp -\frac{|V t|}{l_{c}}
\end{aligned}
$$

Now we can substitute these expressions into equations 7.4 and calculate the particle diffusivity. In particular, in the limit of long times

$$
\lim _{t \rightarrow \infty} D(t)=\int_{0}^{\infty} R(y) d y
$$

and the long-time diffusivity is given in the vertical direction by

$$
D^{(I I)}=\frac{1}{3} u^{2}\left[\frac{t_{L} \ell_{c}}{\lambda \ell_{c}+|v| t_{L}}\right]
$$

and in the horizontal plane by 
$D^{(1)}=\frac{1}{3} u^{2}\left[\frac{t_{L}{ }^{2} c}{\lambda l_{c}+|v| t_{L}}\right]\left[1-\frac{|V| t_{L}}{2\left(\lambda \ell_{c}+|V| t_{L}\right)}\right]$

We can relate these results to the passive scalar diffusion

$$
D_{F}=\frac{1}{3} \overline{u^{2}} t_{L}
$$

by using the relation

$$
t_{L} \cong \frac{\ell_{c}}{\sqrt{\frac{u^{2}}{u^{2}}}}
$$

(Csandy $^{27}$, p.54, Snyder and Lumley ${ }^{15}$ ). Then we can write

$$
\begin{aligned}
& D^{(I I)}=D_{F}\left(\frac{1}{\lambda+\frac{[v]}{\sqrt{u^{2}}}}\right)
\end{aligned}
$$

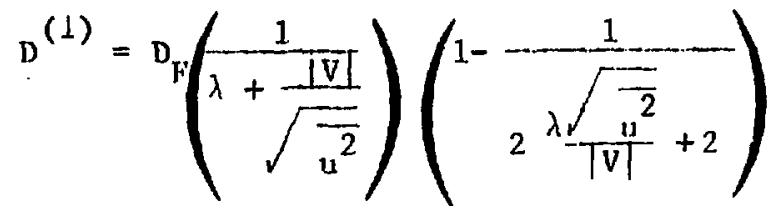

Note that $\mathrm{D}^{(1)}<\mathrm{D}^{(l l)}$. Since the quantity $\frac{v}{\sqrt{\mathrm{u}^{2}}}$ is assumed large

and $\lambda$ is of order ten, we see that the long-time diffusion coefficient in the case of raindrops falling through the air is much smaller than the passive scalar diffusion coefficient. 
We have been unablo to find any experimental data specifically relating to raindrops. However, equations 7.6 should be applicable to any particle through a turbulent fluid so long as the terminal velocity of the particle relative to the fluid is large compared to the ront-meansquare flurtuating fluid velocity. Certain of the particles in the experiment of Snyder and Lumley ${ }^{1.5}$ satisfy this condition, and for these particle wo can compare equations 7.6 with measured data.

Two types of particles used in this experiment were glass beads of density $2.5 \mathrm{~g} \mathrm{~cm}^{-3}$ and diameter $8710^{-4} \mathrm{~cm}$, and copper beads of density $8.9 \mathrm{~g} \mathrm{~cm}^{-3}$ and diameter $46.510^{-4} \mathrm{~cm}$. These particles were Injected into a turbulent flow of air which noved vertically upwards. The Reynolds number of relative flow in the experiment is of order one, and so the terminal velocity should be calculated by balancing the acceleration due to gravity against the deceleration due to Stokes diag. The terminal velocity is given by

$$
v=\frac{2 \rho_{p} R_{o}^{2} g}{9 v \rho_{F}}
$$

For the glass beads, this is $68.6 \mathrm{~cm} \mathrm{sec}-1$ and for the copper beads is $69.8 \mathrm{~cm} \mathrm{sec}^{-1}$. The quantity $\sqrt{\mathrm{u}^{2}}$ in the experiment was measured to be $7.9 \mathrm{~cm} \mathrm{sec}{ }^{-1}$, which is much smaller than the terminal velonity. The experimental particle diffusivities can be ralculated from figure 9 of the experimental paper with the relation

$$
D=\frac{1}{2} \frac{d \overline{Y^{2}}}{d t}
$$

and are $1.48 \mathrm{~cm}^{2} \mathrm{sec}^{-1}$ for the glass beads and $0.41 \mathrm{~cm}^{7} \mathrm{sec}^{-1}$ for the 
copper beads. The passive scalar diffusivity is estimated in the experiment by Snyder and Lumley as $\sqrt{u^{2}} l_{\text {u }}$ which is $36.2 \mathrm{~cm}^{2} \mathrm{sec}^{-1}$. Finally. the quantity $\lambda$, the constant which relates the Lagrangian and Eulerian time scales, is measured in the experiment and found to equa] 3.

Realizing that the experiment measured displacemonts in the horizontal direction perpendicular to the direction of mean flow, we can compare the theoretical prediction

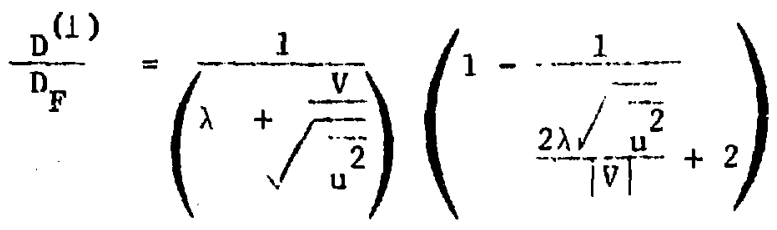

with the experimental data, with the following results

\begin{tabular}{|l|c|c|} 
& ${\frac{D}{D_{F}}}^{(1)}$ (theory) & ${\frac{D}{D_{F}}}^{(1)}$ (experiment) \\
\hline glass & .053 & .041 \\
copper & .052 & .039
\end{tabular}

TABLE 7.1

The agreement between theory and experiment, for these two types of particles appears to be quite satisfactory. The theory predicts that the diffusivity for these two types of particles is nearly the same, despite the differences in density and radius. The theory also predicts that 
the glass beads have a slightly larger diffusivity than the copper beads which also agrees with the measured data. The predicted numbers differ by about $20 \%$ from the measured numbers. One could explain this on the following basis. In the experiment, the quantity $\lambda$ is estimated by using the measured correlation time of the copper beads as the Eulerian integral scale, and the correlation time of hollow glass beads (density $.26 \mathrm{~g} \mathrm{~cm}^{-3}$, diameter $46.510^{-4} \mathrm{~cm}$ ) as the Lagrangian integral scale. In this way, the Lagrangien scale is underestimated and the Eulerian scale overestimated, and so $\lambda$ is $1 \mathrm{ikely}$ underestimated. If we instead use the value $\lambda=10$ suggested by Lumley and Panofsky ${ }^{26}$, the theory would lead to

$$
\begin{aligned}
& \frac{D^{(1)}}{D_{F}}=.041 \quad \text { (glass beads) } \\
& \frac{D^{(1)}}{D_{F}}=.041 \quad \text { (copper beads) }
\end{aligned}
$$

This would represent excellent agreement between theory and experiment. 


\section{REFERENCES}

1. A. Einstein, Theory of the Browntan Movement (Dover Publications, Inc., New York, 1956).

2. S. Chandrasekhar, "Stochastic Problems In Physics and Astronomy," Rev. Mod. Phys. 15, 1-89 (1943).

3. C. M. Tchen, "Mean Value and Correlation Problems Connected with the Motion of Small Particles Suspended in a Turbulent Fluid,"

Ph. D. thesis, De1ft (1947).

4. J. O. Hinze, Turbulence (McGraw-Hi11 Book Company, Inc. New York, 1975) 2nd Ed.

5. A. S. Monin and A. M. Yaglom, Statistical Fluid Dynamics (The MIT Press, Cambridge, 1971) Vo1. I.

6. v. C. I.Iu, "Turbulent Dispersion of Dynamic Particles," J. Metero1. 13. 399-405 (1956).

7. V. G. Levich and S. I. Kuchanov, "Motion of Particles Suspended in Turbulent Flow," Soviet Physics Doklady 12, 546-548 (1967).

8. S. K. Friedlander, "Behavior of Suspended Particles in a Turbulent F1uid," A.I.Ch.E. Journa1 3, 381-385 (1957).

9. S. Corrsin and J. Lumley, "On the Equation of Motion for a Particle In a Turbulent Fluid," Appl. Sc1. Res. 6A, 114-116 (1956).

10. J. O. Hinze, "Turbulent Fluid and Particle Interaction," Progress in Heat and Mass Transfer, 6, 433-452 (1971).

11. 3. L. Lumley, "Two-Phase and Non-Newtonian Flows," In Turbulence, P. Bradshaw, Ed. (Springer-Verlag, New York, 1976).

12. R. L. Peskin, "Some Effects of Particle-Particle and Particle Fluid . Interaction in Two Phase Flow Systems," Ph.D. thesis, Princeton University (1960).

13. v. N. Goldschmidt, M. K. Householder, G. Ahmadi and S. C. Chuang, "Turbulent Diffusion of Small Particles in Turbulent Jets," Progress in Heat and Mass Transfer, 6, 487-508 (1971).

14. J. L. Lumley, "Some Problems Connected with the Motion of Sma11 Particles in a Turbulent Fluld," Ph.D. thesis, The John Hopkins University (1957). 
15. W. H. Snyder and J. L. Lumley, "Some Measurements of Particle Veloctty Antocorrelation Functions In a Turbulent Flow, J. Fluid Mech. 48, 41-71 (1971).

16. R. L. Peskin, "Stochastic Estimation Applications to Turbulent Diffusion," Proceedings of the International Symposium on Stochastic Hydraulics, Pp. 251-267 (1971).

17. A. A. Sveshnikov, Applied Methods of the ineory of Random Functic.s (Pergamon Press, New York, 1966) pp. 2.5-31.

18. Francis H. Harlow, and Anthony A. Amsden, "Flow of Interpenetrating Material Phases," J. Comp. Phys. 18, 440-464 (1975).

19. L. D. Landau and E. M. Lifshitz, Fluid Mechanics (AddisonWesley Publishing Company, Inc., Reading, Massachusetts, 1959).

20. T. Pearcey, and G. W. Hill, "The Accelerated Motion of Droplets and Bubbles," Australian J. Phys. 9, 19-30 (1955).

21. G. E. Uhlenbeck, and L. S. Ornstein, "On the Theory of Brownian Motion," Phys. Rev. 36, 823-841 (1930).

22. A. T. Hjelmfelt, and I. F. Mockros, "Motion of Discrete Particles in a Turbulent Fluid:" App1. Sci. Res. 16, 149-161 (1966).

23. Konrad Knopp, Theory of Furictions, Part I (Dover Publications New York, 1945), pp. 85-91.

24. Sir Horace Lamb, Hydrodynamics (Dover Publications, New York, 1945).

25. F. Pasquil1, Atmospheric Diffusion (John Wiley and Sons, New York, 1974).

26. John L. Lumley, and Hans A. Panofsky, The Structure of Atmospheric Turbulence (Interscience Pub!jshers, New York, 1964).

27. G. T. Csanady, Turbulent Diffusion in the Environment (D. Reide1 Pub1ishing Company, Boston, 1973). 


\section{\CKNOWLEDGEMENTS}

I wish to thank Frank Harlow of the Los Alamos Scientific Laboratory and David Williams of The University of Michigan for their help and patience during the writing of this thesis. Thanks are also due to Jean Romero and Juanita Salazar for typing the firal draft. 